\title{
Investigations of the auroral luminosity distribution and the dynamics of discrete auroral forms in a historical retrospective
}

\author{
Y. I. Feldstein ${ }^{1}$, V. G. Vorobjev ${ }^{2}$, V. L. Zverev ${ }^{2}$, and M. Förster ${ }^{3}$ \\ ${ }^{1}$ Institute of Terrestrial Magnetism, Ionosphere, and Radiowave Propagation of the Russian Academy of \\ Sciences (IZMIRAN), 142090 Troitsk, Moscow region, Russia \\ ${ }^{2}$ Polar Geophysical Institute, Apatity, Murmansk region, 184209, Russia \\ ${ }^{3}$ GFZ German Research Centre for Geosciences, Helmholtz Centre Potsdam, 14473 Potsdam, Germany \\ Correspondence to: Y. I. Feldstein (aifeld@aol.com)
}

Received: 18 December 2013 - Revised: 17 March 2014 - Accepted: 16 April 2014 - Published: 21 May 2014

\begin{abstract}
Research results about planetary-scale auroral distributions are presented in a historical retrospective, beginning with the first "maps of isochasms" - lines of equal visibility of auroras in the firmament (Fig. 2) - up to "isoaurora maps" - lines of equal occurrence frequency of auroras in the zenith (Fig. 4). The exploration of auroras in Russia from Lomonosov in the 18th century (Fig. 1) until the start of the International Geophysical Year (IGY) in 1957 is shortly summed up. A generalised pattern of discrete auroral forms along the auroral oval during geomagnetically very quiet intervals is presented in Fig. 5. The changes of discrete auroral forms versus local time exhibit a fixed pattern with respect to the sun. The auroral forms comprise rays near noon, homogeneous arcs during the evening, and rayed arcs and bands during the night and in the morning. This fixed auroral pattern is unsettled during disturbances, which occur sometimes even during very quiet intervals. The azimuths of extended auroral forms vary with local time. Such variations in the orientation of extended forms above stations in the auroral zone have been used by various investigators to determine the position of the auroral oval (Fig. 9). Auroral luminosity of the daytime and nighttime sectors differ owing to different luminosity forms, directions of motion of the discrete forms, the height of the luminescent layers, and the spectral composition (predominant red emissions during daytime and green emissions during the night). Schemes that summarise principal peculiarities of daytime luminosity, its structure in MLT (magnetic local time) and MLat (magnetic latitude) coordinates, and the spectral composition of the luminosity are presented in Figs. 15 and 19. We discuss in detail the daytime sector dynamics of individual discrete forms for both quiet conditions and auroral substorms. The most important auroral changes during substorms occur in the nighttime sector. We present the evolution of conceptions about the succession of discrete auroral forms and their dynamics during disturbance intervals. This ranges from Birkeland's polar elementary storms, over the prospect of a fixed auroral pattern up to the auroral substorm model. The classic schemes of the spatial distribution and motion of discrete auroral forms during single substorms are shown in Fig. 20 (expansive and recovery phases) and Fig. 21 (creation, expansive and recovery phases). In this review we discuss various models of bulge formation, in particular as a result of new formation of arcs about $50-100 \mathrm{~km}$ poleward of previously existing auroral structures (Fig. 24). Discrete steps in the development of an expanding bulge are separated by 1-3 min from each other. The model of successive activations confines only to a $\sim 40^{\circ}$ longitudinal portion of the magnetotail (Fig. 28). We consider differences in the development of single substorms and substorms during magnetic storms. The structure and dynamics of auroras during steady magnetospheric convection (SMC) periods are dealt with in Sect. 8. A generalised scheme of the auroral distribution during SMC periods is shown in Fig. 34. Separate sections describe discrete auroras in the polar cap (Sect. 5), and the diffuse luminosity equatorward of the auroral oval (Sect. 9). Visual observations of diffuse auroral forms at midlatitudes suggest that the whole latitudinal interval between the auroral oval and the stable auroral red (SAR) arc is filled up with diffuse luminosity. SAR arcs with intensities of several tens of Rayleigh enclose systematically the region of diffuse luminosity; they are positioned at the border of the plasmasphere.
\end{abstract}


1

\section{Introduction}

The aurorae, which appear as a conspicuous play of colours, a wide changeability of forms and fast variations of luminosity, have always attracted the fascination and amazement of people that entered the Arctic or Antarctic circle of latitudes for the first time. Bright forms of aurorae appear during nighttime hours most likely at geomagnetic latitudes of $\sim 67-75^{\circ}$. During geomagnetic storms the region of its appearance broadens, comprising midlatitudes as far as the Mediterranean area. Written records verify that mankind encountered this phenomenon already in ancient times. Aurorae borealis are mentioned in the Bible (Genesis) and in the oeuvres of ancient philosophers and historians such as the Greeks Aristotle and Plutarch as well as the Romans Pliny the Elder and Seneca.

The luminescence in the upper atmosphere, emerging from the precipitation of corpuscular fluxes, can appear as a structureless diffuse luminosity or as clear cut structures. The diffuse luminosity is observed from the geomagnetic pole to midlatitudes and is generated by different corpuscular sources: (1) the homogeneous luminescence in the polar cap is due to the precipitation of solar protons with energies of several hundreds of megaelectronvolts or electron fluxes with energies less than $1 \mathrm{keV}$ (polar rain) from parts of the magnetospheric tail (tail lobe) and/or the solar wind; (2) diffuse luminosity due to the precipitation of protons or electrons with auroral energy (between a few dozen electronvolts and several kiloelectronvolts) at auroral latitudes; and (3) diffuse luminosity due to soft electron precipitations at midlatitudes, including an intensification of red emissions near the plasmapause.

The optical features resulting from the interaction of magnetospheric plasma with the neutral gases in the upper atmosphere represent the most spectacular manifestation of physical processes in Earth's environment. Their study plays an important role for the description and interpretation of the complexities inherent in the coupled physical processes of the near-Earth space. The brightest discrete forms of auroras are generated at auroral latitudes owing to precipitating electrons with energies of several kiloelectronvolts during nighttime hours and with energies of several hundred electrovolts during daytime hours. Discrete auroral forms can also be generated within the polar cap, but their morphology essentially differs from that at auroral latitudes.

Auroral luminosity that is observed along the auroral oval during nighttime hours is usually of green colour. As stated for the first time by Angström (1869), this emission of the atomic oxygen with a wavelength of $557.7 \mathrm{~nm}$ is due to the forbidden transition of electrons from the ${ }^{1} \mathrm{~S}$ state $(4.17 \mathrm{eV})$ with a lifetime of $0.9 \mathrm{~s}$ to the ${ }^{1} \mathrm{D}$ state $(1.96 \mathrm{eV})$. This wavelength is close to the most sensitive range of the human eye, which is the reason for dominance of green light in the visual impression. A red light emission dominates during daytime hours along the auroral oval, which is due to the doublet of atomic oxygen with 630.0 and $636.4 \mathrm{~nm}$, where the exited state relaxes to the ground state with a lifetime of $110 \mathrm{~s}$. The difference in the colours of daytime and nighttime luminosity along the auroral oval is correlated with different altitudes of the emissions. The red emission is more intense than the green one at altitudes above $\sim 200 \mathrm{~km}$, while the green emission prevails at $\sim 110 \mathrm{~km}$. The height difference is caused by harder spectra of precipitating electrons on the nightside as well as by a stronger collision deactivation of the red emission at lower altitudes prior to its radiation.

The fluxes of energetic electrons are guided by the geomagnetic field lines in their downward motion into the atmosphere. They suffer elastic and inelastic collisions, transfer their energy to the neutral particles during processes of dissociation, ionisation, and excitation with subsequent emissions and give likewise rise to atmospheric heating. The penetration height of the electron fluxes is determined by their energy and pitch-angle distribution. According to model calculations, electrons that have an initial pitch angle of $0^{\circ}$ (initial movement straight along the field line) and an energy of $\sim 100 \mathrm{eV}$ penetrate down to altitudes of $\sim 300 \mathrm{~km}$, and those with $5 \mathrm{keV}$ down to $\sim 110 \mathrm{~km}$ (Judge, 1972). The lower boundary of penetration rises with increasing pitch angles.

The altitude profile of the ionisation production rate is characterised by a distinct maximum slightly above the lower boundary of electron flux penetration (Strickland et al., 1983). The modelling of altitude profiles of volume emission rate ratios of $6300 \AA / 5577 \AA$ by Rees and Luckey (1974) demonstrated the possibility to use it for the experimental determination of the emission height. It turned out that this ratio increases systematically from $\sim 0.1$ for a height of $140 \mathrm{~km}$, to $\sim 0.5$ at $200 \mathrm{~km}, \sim 2.0$ at $260 \mathrm{~km}$, up to $\sim 5.0$ at $300 \mathrm{~km}$.

The intensity of auroral luminosity is determined by the energy flux of the primary corpuscular flux: the green emission in $\mathrm{erg} \mathrm{cm}^{-2} \mathrm{~s}^{-1}$ intensity of $\sim 1-30 \mathrm{kR}$ is due to fluxes of $1-30 \mathrm{erg} \mathrm{cm}^{-2} \mathrm{~s}^{-1}$. The red emission in the daytime sector is several times weaker and induced by fluxes of $\sim 1 \mathrm{erg} \mathrm{cm}^{-2} \mathrm{~s}^{-1}$.

A classification of auroras, i.e. the luminosity within the upper atmosphere that is controlled by the geomagnetic field, has been presented in the International Auroral Atlas (1963) and in the paper of Isaev (1964). Examples of various auroral forms are shown in the colour Plates I-VII with photographs taken near Murmansk at the Kola Peninsula during nighttime.

Ribbons, rays, and diffuse luminosities are three types of auroras which are chosen in the scientific literature. Arcs and bands are counted among ribbon-like forms. Arcs are characterised by a sharply defined lower border. Multiple arcs with intervals of $30-40 \mathrm{~km}$ are met very often. If and when the lower border of an aurora appears erratically or contains 

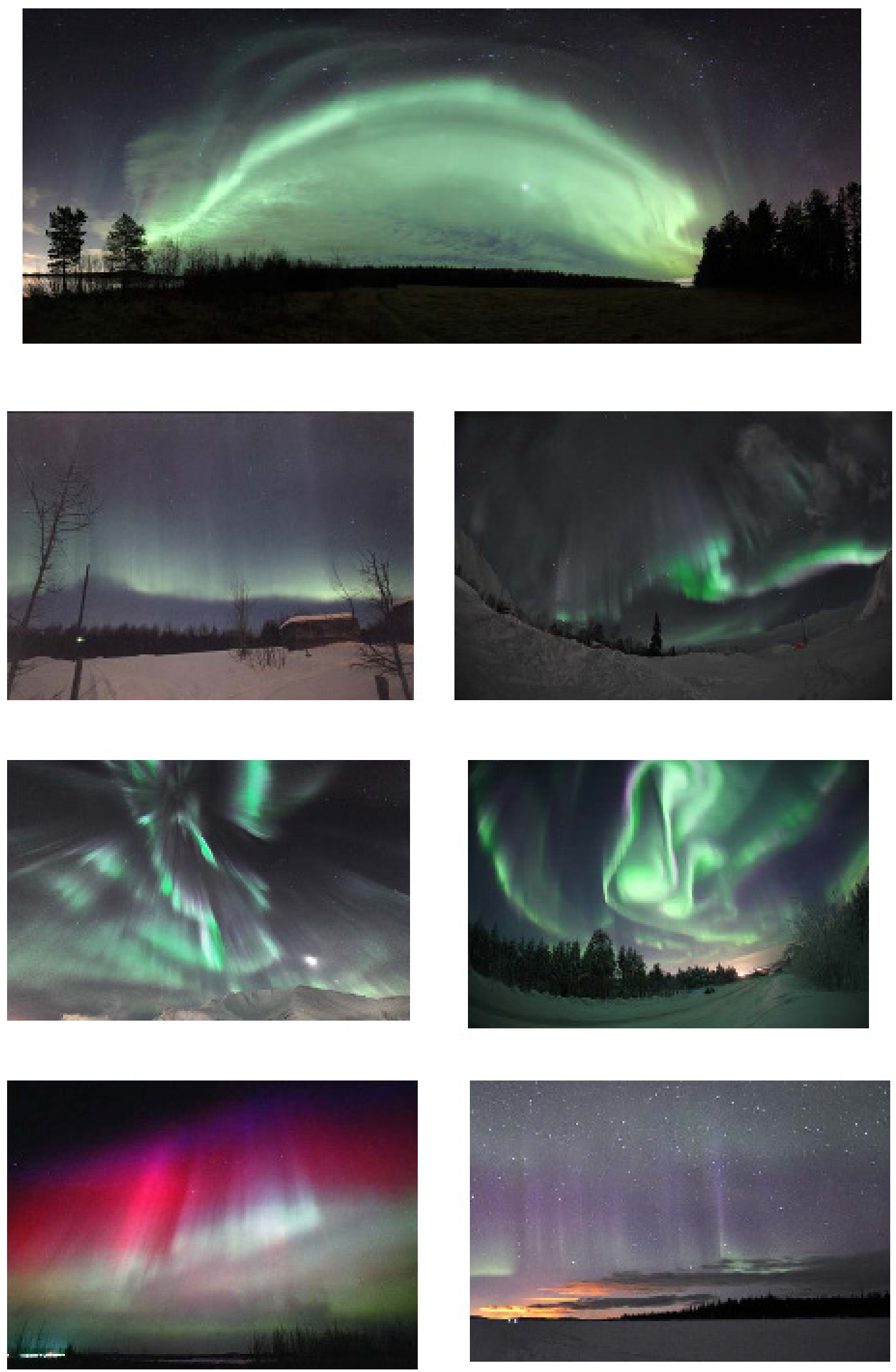

Plates I-VII. Photos of various auroral types, taken at the Kola Peninsula by S. A. Chernouss and V. Yu. Zhiganov (Apatity, Russia). From top to bottom: (I) Homogeneous auroral arc, (II) rayed arc, (III) long auroral rays, (IV) corona - emissions along the magnetic field lines, (V) drapery - ribbon-like auroral forms, (VI) simultaneous green and red auroral emissions at different heights, and (VII) sunlit aurora. 
bulges or surges, this auroral form is called a band. Bands are usually more active than arcs.

Ribbon-like aurorae spread along the auroral oval over several thousand kilometres and in the perpendicular direction up to several hundred kilometres. Arcs, for instance, can spread over distances of $5000-6000 \mathrm{~km}$ and more. The ribbon itself has a typical thickness of several hundred metres, so that one can conclude that luminosity of this kind must be excited by quite narrow electron beams. The ribbonlike forms often look like densely folded curtains, covering closely to one another and stretching over the whole firmament.

When the ribbon becomes somehow more active and moving it forms tiny surges of a few kilometres in thickness. Such a form of luminosity seems to consist of individual stretched rays or light beams; in this case it is called "rayed arc". If and when fine-structured surges or beams lay on top of larger foldings, such forms are called a "rayed band". A salmonorange coloured brink or fringe emerges at the lower part of the rayed band surges with increasing activity.

The diffuse auroral forms can have the appearance of blurred spots that remind of illuminated clouds or a hazy view. Such haze represents an extended homogeneous glow, which often covers most part of the sky. Spots usually form in the auroral zone during the recovery phase of a disturbance.

Rays are narrow beams of light, which are spatially adjusted along Earth's magnetic field lines. Bundles of rays can be observed, which are either close to each other or dispersed. Rays are often observed together with other forms of auroral luminosity. The most impressive auroral form, the "corona", emerges in case of overhead appearance of the rays in the magnetic zenith.

This review is devoted to several aspects of investigating the morphology of auroral luminosity in a historical retrospective. Its scope also aims at an extensive explanation of results that are covered in scientific publications in the Russian language. Such information will be of interest and useful for many of those scientists who have problems either due to the language barrier or because of undue hardship to obtain such information, which are buried in institute collections or in particular topical proceedings.

\section{A look at the Russian history}

The first written records in Old Russia (Muscovy) about auroras go back to the 10th century. These phenomena were described as events that must be followed by disasters and adverse weather. But already in chronicles of the 16th century, the auroras are more correctly described as a certain natural phenomena. In one of the ancient Russian manuscripts (from 1586 to 1600), the auroras (northern lights) are explained as reflections of the solar light from the wavy surface of the northern seas. Later, in the mid-17th century, Descartes formulated a similar assumption. The evolution of opinions about the aurora borealis in the Russian literature and science from the 10th to the 18th century was summarised in the work of Swjatskij (1934).

The term "aurora borealis" was introduced in the Russian literature by the outstanding Russian scientist Mikhail Vasilyevich Lomonosov (1711-1765) in the 1740s. The year 2011 was declared by the UNESCO as the International Year of Lomonosov in honour of his 300th anniversary. When it became clear that the auroras are also observed in the Southern Hemisphere, the term "polar aurorae" got used. Hypotheses about the origin of polar lights were manifold. But almost all of them that refer to previous centuries are nowadays only of historical interest because of their ingenuousness. M. V. Lomonosov annotated his view about such hypotheses in his poetic ode The evening reflection about God's majesty during the case of great polar lights, written in 1743.

Figure 1 shows a few drawings of different auroras, carried out by M. V. Lomonosov after observations in northern Russia. Forty seven of such drawings are kept in the M. V. Lomonosov Museum in St Petersburg.

Lomonosov (1753) and afterwards Franklin (1779) were apparently the first scientists who expressed the view about an electrical origin of polar lights. Lomonosov assumed that the polar auroras are in their nature essentially equal to lightning, which is observed during electrical discharges. He formulated this idea first in his discourse at the session of the Academy of Sciences in 1753 on Consideration about aerial phenomena, caused by electrical forces (Oratio de meteoris vi electrica ortis). He wrote in his treatise on physics (17531756): "Adeo igitur probabile est, aurorae borealis lumen producta in aëre vi electrica oriri. Confirmatur hoc similitudine generationis et interitus, motus, coloris, et figurae, quae in aurora boreali et in lumine electrico tertii generis conspiciuntur." (Thus it is very likely that the aurorae borealis are generated by electric forces occurring in the air. This is confirmed by the similarity in appearance and disappearance, movements, colours and forms, which in aurora borealis and in the electric light of third nature is observed.) (Lomonosov, 1952).

An important question relating to the reasons for aurora generation is the determination of its height. In difference to most of the other scientists, M. V. Lomonosov supposed that the auroras occur at high altitudes above the atmosphere. $\mathrm{He}$ performed a calculation of the upper edge of the luminescence and obtained 420 verstas $(\sim 440 \mathrm{~km})$. Several centuries later, Størmer (1955) estimated this height sufficiently precisely by means of photographic auroral observations from two points. He showed that the height of the luminescence is located in the range of $100-400 \mathrm{~km}$. The estimations of M. V. Lomonosov appeared to be close to this result. More detailed studies on the height distribution of daytime and nighttime auroras were performed during the International Geophysical Year (IGY, 1957-1958) by means of photographic all-sky cameras (Starkov, 1968; Khorosheva and Emelyanenko, 1970). 

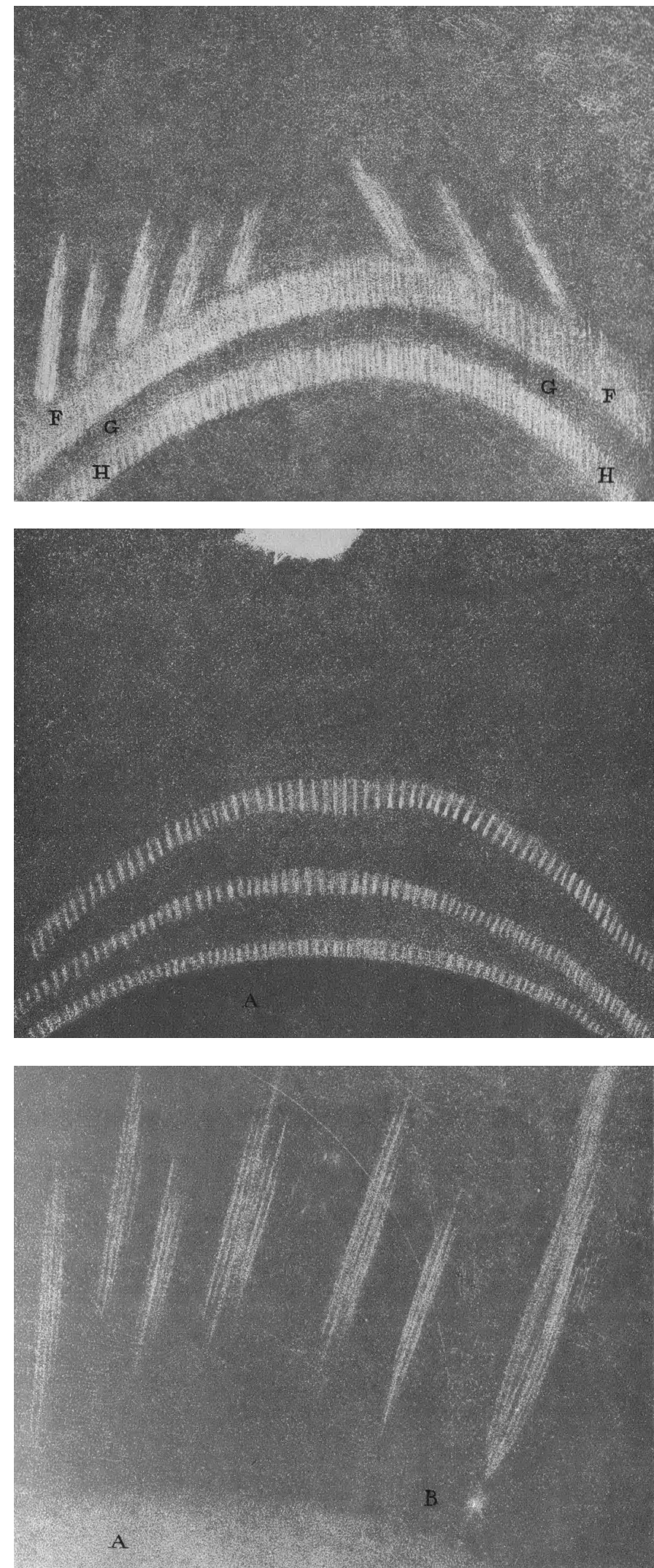

Figure 1. Drawings of various auroral types by Mikhail Vasilyevich Lomonosov. 


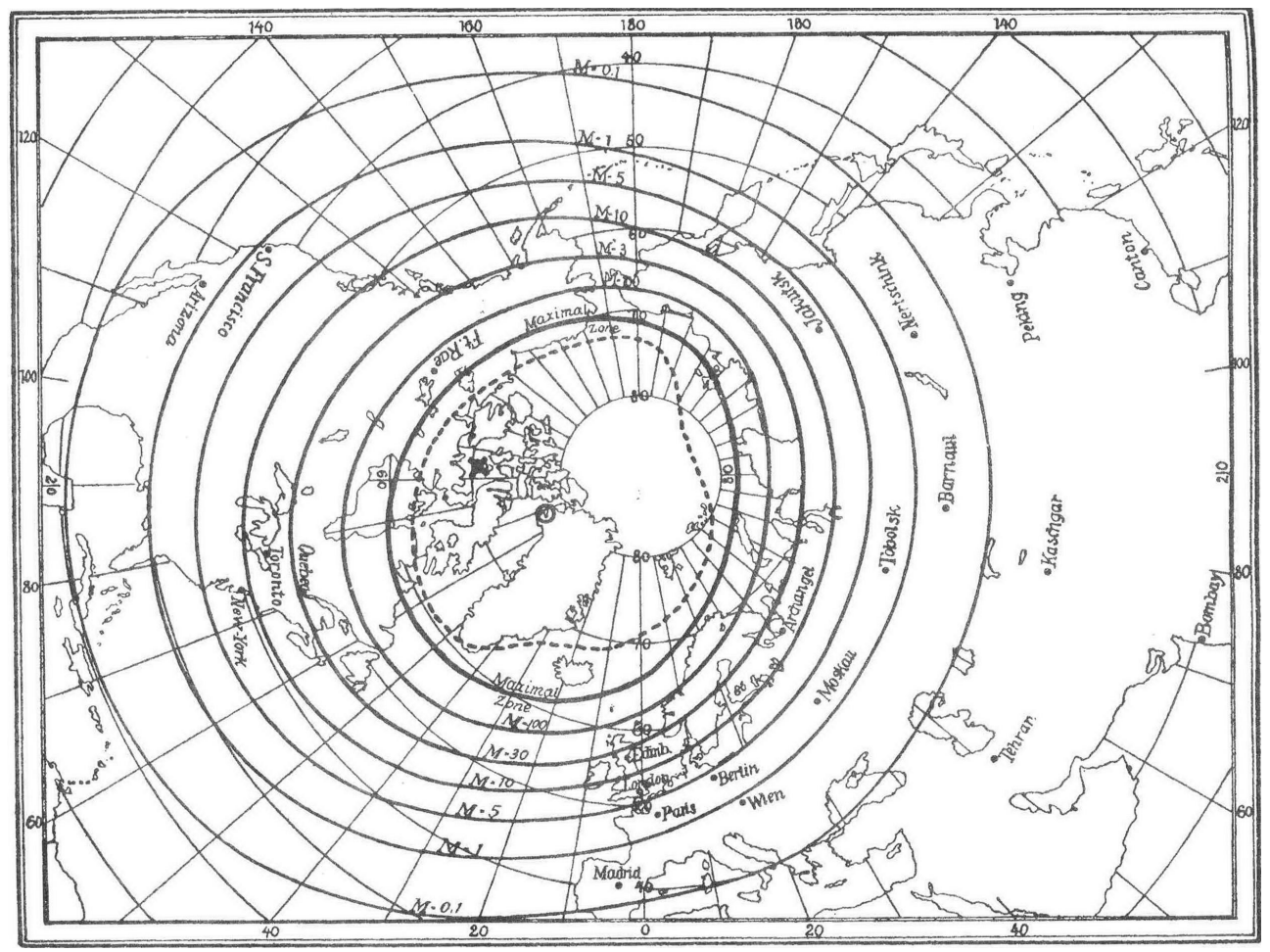

Figure 2. The distribution of isochasms in the Northern Hemisphere according to Fritz (1881).

M. V. Lomonosov carried out a large number of experiments to study the luminescence of discharging gases, when they are subdue to an electrical current flow. Lomonosov's construct of ideas in his investigations of northern lights were described in the paper of Chernouss (2012). His idea about a connection between the colour of the luminescence and certain specific materials turned out to be of practical interest. This speculation of the scientist was later confirmed by direct measurements, which were performed by the well-known Swedish physicist Anders Jonas Ångström with his visual spectroscope (Ångström, 1869). Some years later, in 1899, an astronomer from the Pulkovo Observatory near St Petersburg, Julius Sykora, was the first who took photos of auroras at the Spitzbergen Archipelago (Svalbard), and obtained also for the first time their spectra that characterise the luminescence of different atoms and molecules (Sykora, 1901). These auroral spectra, which were recorded by J. Sykora, allowed determining not only four basic auroral emissions (391.4, 427.8, 470.9, and $557.7 \mathrm{~nm}$ ), but also nine weaker emissions within the blue and violet parts of the spectra.

Historic information about several researchers and their observations of auroras over the Russian territory after M. V. Lomonosov are contained in the very well illustrated monograph of Eather (1980). The fourth chapter, entitled "Northern lights", describes observational results of Russian polar researchers of the 19th century. Baron Ferdinand von Wrangel (in Russian: Ferdinand Petrovich Vrangel), a wellknown explorer and seaman, member of the Saint Peters- burg Academy of Sciences, who is the eponymous person for the Wrangel Island, which is close to the Chukchi Peninsula, was the first to discover that the aurora borealis is predominantly situated along the shoreline of the Arctic Sea (Wrangel, 1840). A possible influence of the coastline on the frequency of auroras and on their orientation was also witnessed with the observations of captain Baron Eduard Vasilyevich Toll during his expedition on the Zarya vessel in 1900-1902.

The famous polar researcher Adolf Erik Nordenskiöld observed the auroras in 1878-1879 during the drift of the Vega vessel and corrected the Fritz auroral zone near the northern coastline of the Chukchi Peninsula (Nordenskiöld, 1881).

The traditions of auroral research continued also in the Soviet Union. In 1938, a special camera for low-temperature conditions was built in the Arctic Institute (St Petersburg) for the auroral photography. The recordings of aurora borealis events were made at the archipelago Franz Josef Land (station Tikhaya Bay, $\Phi^{\prime} \sim 74.4^{\circ}$, Nikolsky, 1939). Leading researchers in the public perception of this natural phenomenon during the years prior to and just after World War II were S. I. Isaev, V. I. Krasovsky, A. I. Lebedinsky, and N. V. Pushkov.

Sergei Ivanovich Isaev (1906-1986), as an active participant of the second International Polar Year (IPY, 19321933), performed observations of aurorae borealis and magnetic field variations at the Novaya Zemlya Island. Later he generalised the data obtained at Soviet polar observatories 


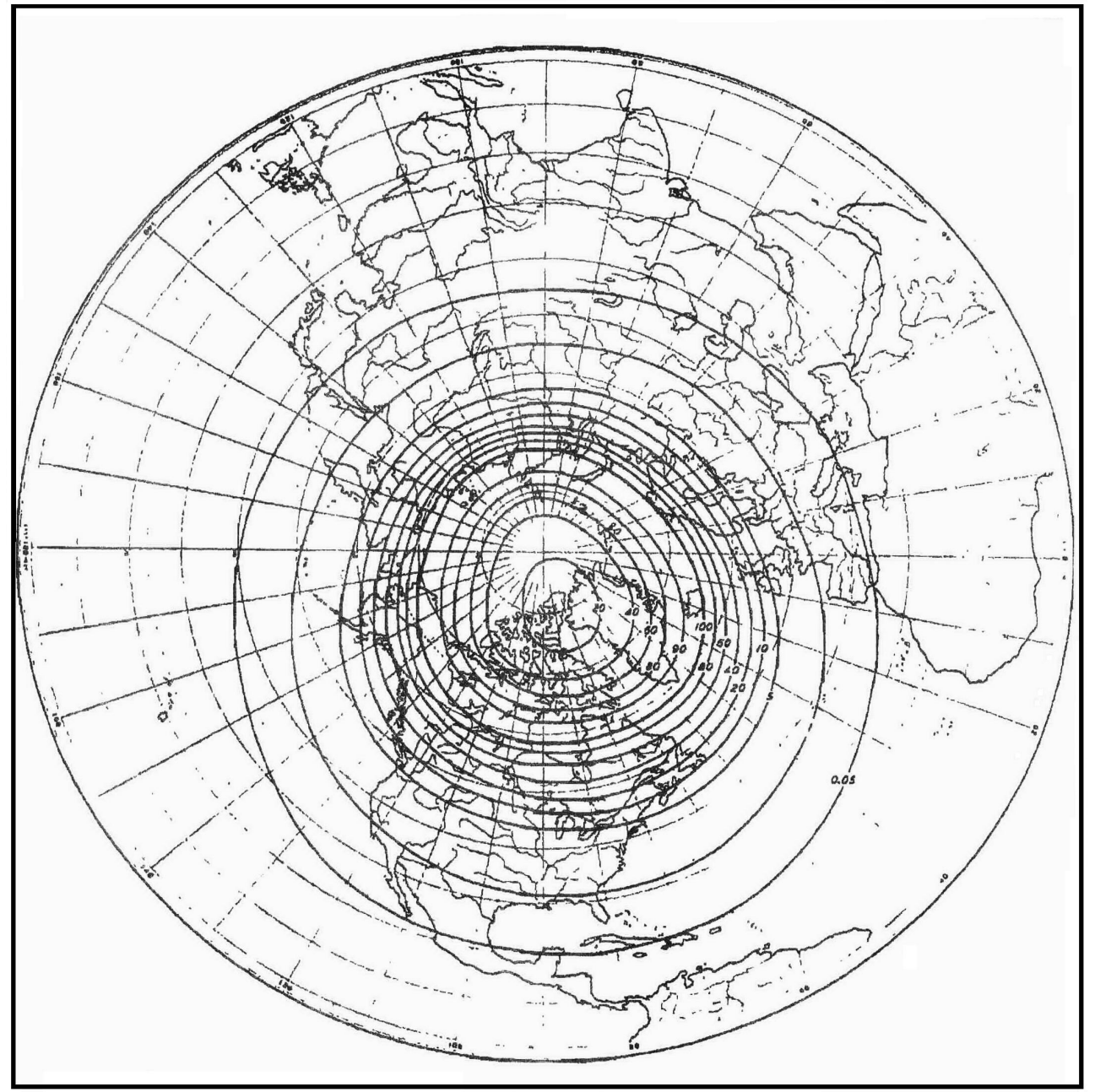

Figure 3. Drawings of isochasms in the Northern Hemisphere according to Vestine (1944).

and concurrently during the drifts of the Sedov and Malygin vessels in the northern Arctic Sea (Isaev, 1939a, b, 1940). Isaev supposed that one has to distinguish between three auroral regions: the main zone of auroral luminosity (a ring of usually $23^{\circ}$ radius), an outer and an inner zone. Each of these zones has its distinctive characteristic. The main zone luminosity appears every day and has a weak dependence on solar activity. In the outer zone the frequency of auroral appearance strongly depends on solar activity, while in the inner zone auroras appear relatively seldom, independently of the solar activity. Isaev also established a generalised scheme for the diurnal periodicity of auroral appearance within the different zones.

Based on visual observations of about 450 stations during the years 1932-1948, Isaev (1962) analysed the geographic distribution of auroras in dependence on geomagnetic activity. He found that a region of enhanced auroral luminosity clearly appears during magnetic storms in the midlatitude range of $\Phi=52-58^{\circ}$, which coincides with the region whereon the outer radiation belt should map along geomagnetic field lines.

Valerian Ivanovich Krasovsky (1907-1993) happily joined in his own person both an ingenious design engineer of new instruments and a prudent scientist who theoretically comprehended the obtained results. He constructed an infrared camera, which was applied both in astronomical and geophysical research. In 1948 he captured a map of the galactic centre and its spiral structure by recording the first infrared all-sky image. The photochemical theory of hydroxyl emission, developed by him, established a new branch of geophysical research. He contributed to a series of newly developed diffraction spectrometers, which are useful for recording weak emissions. $B_{y}$ means of them, new findings about the luminosity in the upper atmosphere were obtained, and it allowed to study in more detail the auroral luminescence over a network of stations during the IGY and later on, e.g. during the International Geophysical Cooperation (IGC) in 1959, an extension of the IGY. He compiled an 

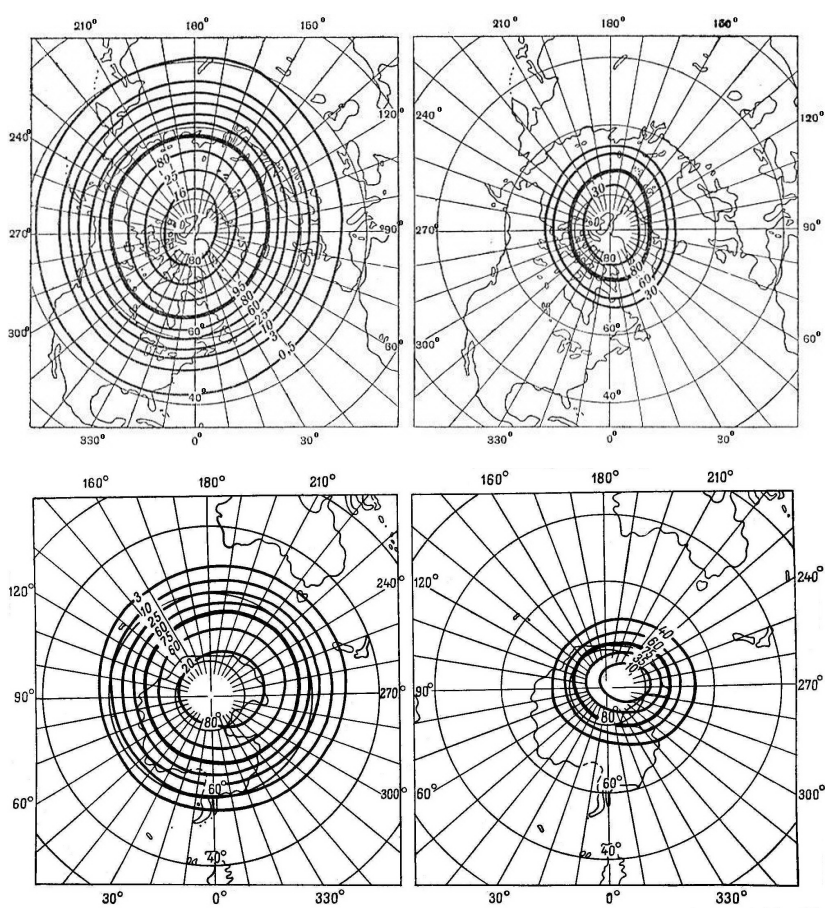

Figure 4. System of "isoaurora" lines for both the Northern (top panels) and Southern Hemispheres (bottom panels). The left-hand side panels show their distribution during nighttime hours, the righthand side panels correspondingly those for daytime hours (Feldstein, 1963a).

atlas of the atmospheric luminescence spectrum in the range of 3000-12400 $\AA$ for the nighttime sky (Krasovsky et al., 1962).

The development of Soviet auroral research is closely connected with the name of Alexander Ignatjevich Lebedinsky (1913-1967). Originally an astronomer, he started to work as auroral researcher in 1948. In the years 1948-1950 he performed observations of aurorae borealis at the Kola Peninsula. During this period he newly developed, built, and applied a specific equipment for the automatic 1 min optical recording of the whole sky and the simultaneous spectral analysis of auroras. Equipment of this kind (the cameras C180 and C-180 S) were widely used in dozens of stations both in the Arctic and Antarctic according to the programs IGY, IGC, and IQSY (International Quiet Sun Year). The subsequent analysis of all the observational materials from the global-scale network fundamentally changed the traditional view of the space-time distribution of auroras.

Nikolay Vassilievich Pushkov (1903-1981) stimulated, with his organisational talent and vigorous working enthusiasm, the transition of the geophysical science in the Soviet Union to a global-scale prospect. He participated very actively in organising the system of World Data Centres, whereof one was installed in Moscow, and he was the first representative of the Interdivisional Commission on History within the International Association of Geomagnetism and

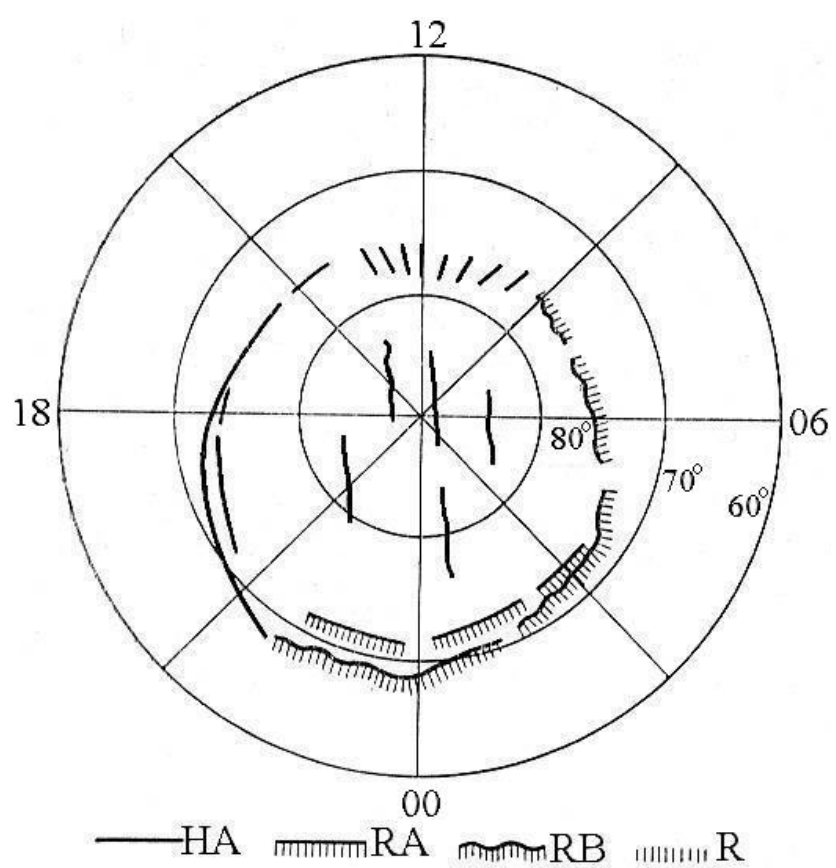

Figure 5. Generalised scheme of discrete auroral forms during quiet intervals according to Feldstein (1966). The labelling is as follows: HA - homogeneous arc, RA - rayed arc, RB - rayed band, and $\mathrm{R}$ - rays.

Aeronomy (IAGA). N. V. Pushkov played a key role for the Soviet Union's participation and organisation of the international program for auroral observations during the IGY and he organised the visual observations in the territory of the Soviet Union and adjacent states. The publication with instructions for visual auroral observations found a broad audience in the beginning of the IGY (Pushkov, 1957). At the 5th IGY Assembly in August 1958, in Moscow, the resolution about the creation of a planetary network for optical observation of auroras was adopted; the so-called visoplot tables, which characterise the latitude, longitude, and UT moments of prevailing auroral luminosities. A complete compilation of planetary visoplots was published in Annals of the IGY (1964).

\section{Planetary distribution of discrete auroral forms}

A first reliable scientific knowledge about the planetary-scale distribution of auroras was obtained during the second half of the 19th century. Loomis (1860) concluded, from historic records of polar lights and from observations of them in New England, that most days of auroral luminosity were reported within an annular belt of $10^{\circ}$ width, encircling both the geomagnetic and the geographic poles. Fritz (1881) collected worldwide in archives a broad knowledge about the appearance of auroras, which served as the basis of his "isochasm" maps, i.e. lines of equal frequency of auroral visibility, as 
shown in Fig. 2. It was disclosed that the quantity of nights with auroras increases when moving from middle toward higher latitudes. The maximum frequency of auroral appearance forms a ring of $\sim 23^{\circ}$ radius on average. The luminosity in this zone (the Fritz auroral zone) is also most intense. The dashed line in Fig. 2 signifies the latitude where the auroras appear equally often within the northern and the southern segments of the sky. The isochasms are almost perfect circles with a common centre. Particularly amazing proved to be the fact that the centre of the isochasms was neither situated at the geographic nor at the magnetic pole, but at the geomagnetic pole. The difference between the magnetic and the geomagnetic poles is caused by the existence of local magnetic anomalies within Earth, the fields of which decay rapidly with height above the surface.

These acquired facts about the existence of auroral zones established for a long time a paradigm of geophysical science. No theory about the generation process of auroras could credibly be designed without explaining the existence of auroral zones. The famous "terrella" experiments of Kristian Birkeland (1867-1917) with a magnetised sphere, irradiated with an electron beam, were initiated by these results.

Based on the materials from the first International Polar Year (IPY, 1882-1883), Vegard (1912) described the tendency of intense discrete auroral forms to appear most often $\sim 1 \mathrm{~h}$ prior to local magnetic midnight. An analysis of the results from the first IPY and of observations in Greenland from 1948 to 1950 was summarised by Lassen (1963) in the following way. There are always two maxima in the diurnal variation of the appearance frequency for discrete auroral forms at high latitudes. At geomagnetic latitudes $\Phi \leq 68^{\circ}$, the main maximum occurs in the hours around midnight, and a less pronounced maximum in the early morning hours. In the magnetic latitude interval $69^{\circ} \leq \Phi \leq 77^{\circ}$ two maxima of equal intensity exist in the morning and evening hours. At $\Phi \geq 78^{\circ}$, the morning maximum dominates close to midday.

Visual auroral observations from the Godthåb $\left(\Phi^{\prime} \sim 73.3^{\circ}\right)$ station in Greenland attracted the particular interest of researchers (Tromholt, 1882). The diurnal variation of the appearance frequency was characterised there by two maxima: in the late evening and in the morning hours, with a minimum shortly after midnight. Hulburt (1931) concluded after a careful analysis of a large amount of observations, however, that a secondary morning maximum is likewise as probable as its absence.

Full-time visual auroral observations are not possible in Greenland, even during wintertime due to solar illumination within the interval 07:00-17:00 LT (local time). This complicates the precise estimation of the morning maximum. Earlier observations of Carlheim-Gyllenskiöld (1887) from the station Cape Thordsen (Spitzbergen, $\Phi^{\prime} \sim 74.3^{\circ}$ ), where full-time observations are practically possible, supported the existence of two maxima - one during the morning, and one during the pre-midnight MLT hours. The morning maximum during the period 05:00-07:00 MLT was interpreted by
Nikolsky (1960) as evidence for the existence of a second frequency maximum of auroras in the polar cap.

More than 60 years after Hermann Fritz' (1810-1882) research had to elapse until new generalised insights were obtained about the distribution of auroras in the Northern Hemisphere (Vestine, 1944). Figure 3 shows the "isochasms" of the Northern Hemisphere according to Vestine. Data about the appearance frequency of auroras from 103 observational points were used, including the records of the Vega (1878-1879), the Fram (1899-1902), and the Maud (1922-1925) vessels during their drifts in the Arctic Sea.

Quite heterogeneous data were standardised beforehand by introducing a correction term, which took account of the cloudiness and the length of daily illumination for each station to estimate the auroral appearance frequency. The area of observational coverage was extended to include the whole polar region, so that the isochasm map could be augmented. Even though the auroras at high latitudes could be recorded practically each night, their appearance frequency dropped to $20 \%$ near the geomagnetic pole.

Auroral data from the Southern Hemisphere, which could be used for the creation of a corresponding isochasm map, were relatively seldom. White and Geddes (1939) were able to determine the position of the maximum isochasms only for the longitude interval $80-280^{\circ} \mathrm{E}$ by collecting observations from expeditions at the Antarctic continent. Vestine and Snyder (1945) also published a map of isochasms for the Southern Hemisphere. The position of maximum isochasms was obtained by taking into account the variations of the geomagnetic field components.

Just prior to the IGY (1957-1958), the geographic distribution of auroras was investigated over a relatively broad area with visual observations at five points in Alaska (Elvey et al., 1955) and at 50 meteorological stations that were distributed over a range of $67.3-87.5^{\circ}$ of geographic latitude and $50-110^{\circ} \mathrm{E}$ geographic longitude (Feldstein, 1958). It was shown that the auroras appear practically daily within this latitudinal zone, both during magnetically quiet and disturbed conditions.

During the IGY, observations of the auroras and their analysis was realised according to an ambitious program. Before the IGY program started, Chapman (1957) formulated the need to construct maps of "isoauroras" instead of "isochasms", which would characterise the appearance frequency of auroras in the zenith of any observational point. The observations at the beginning of the IGY showed the need for a more precise description of the planetary auroral distribution (Nikolsky, 1960). The possibility of splitting the zone of maximum intensity of magnetic variations into an evening and a morning branch was discussed both by Chapman (1935) and Harang (1946). The position of these branches varies with the magnetic disturbance level. Based on the close connection of magnetic variations with the auroras, Harang (1946) proposed the existence of an analogue to the discontinuity zone in the auroral luminosity. Alfvén 
(1955) and Nikolsky (1956) predicted that there should exist a second zone of enhanced intensity and appearance frequency both of auroras and magnetic variations in the nearpolar region. All these predictions and assumptions had to be verified, based on new and more ample observational material of auroras from the IGY.

During the IGY, the main equipment for auroral observations at high latitudes consisted of a network of all-sky cameras (ASCs), which continuously took images of the whole firmament. After completion of the IGY, so-called "ascaplots" (all-sky camera plots) were assembled, based on all the all-sky films (Annals of the IGY, 1962). Ascaplots show for each half-hour interval the spatial distribution of the auroras, and its intensity in the zenith, but also the meteorologic conditions and the sun's illumination interval, which might be adverse for the observations. Feldstein (1960) analysed the results obtained from the ascaplots, comprising 42 Northern and 22 Southern Hemisphere stations. Figure 4 presents according to Feldstein (1963a) the system of isoaurora lines for the Northern Hemisphere (top panels) during nighttime (a) and daytime hours (b) and correspondingly for the Southern Hemisphere (bottom panels, c, d). Maximum isoauroras are indicated by thick lines. It is obvious that the maximum isoauroras are at different latitudes for the daytime and nighttime hours, confirming the conception of two independent auroral zones - the basic zone of Fritz-Vestine and the additional zone of Alfvén-Nikolsky. But in reality such a distribution of maximum isoauroras for the daytime and the nighttime hours at Earth's surface results from the existence of discrete auroral forms along the auroral oval. The oval is (asymmetrically) situated off-centred to the geomagnetic pole - at $\sim 78^{\circ}$ around the noontime hours and at $\sim 67^{\circ}$ near midnight. During the rotation of Earth below the oval, which is fixed with respect to the sun, its daytime sector "draws" the Alfvén-Nikolsky zone at Earth's surface, and the nighttime sector the Fritz-Vestine zone.

The asymmetric distribution of maximum isoauroras with respect to the geomagnetic poles results from the theory of magnetic storms and auroras that dates back to Hannes Alfvén (1908-1995) in the middle of the last century (Alfvén, 1950). These geophysical phenomena originate, according to his theory, from the interaction of the solar corpuscular flux with the near-Earth environment. The flux conserves during its earthward movement the solar magnetic field and an electric field is additionally generated due to polarisation processes of the plasma within the flux. As a result a forbidden region with the ring current is formed at a geocentric distance of $\sim 7 R_{\mathrm{E}}$ in the equatorial plane of the geomagnetic field. The auroral curve that represents the instantaneous position of the aurora is the projection of the forbidden region's boundary along the geomagnetic field lines into the upper atmosphere at a geomagnetic latitude of $\sim 67^{\circ}$. The inertia of the beam was taken into account in subsequent studies by Alfvén $(1955,1958)$. This resulted in the assumption of a second ring current at $\sim 30 R_{\mathrm{E}}$. Its flux can be pro- jected along the magnetic field lines to geomagnetic latitudes of $80-85^{\circ}$ and is associated with the inner (second) auroral zone and magnetic disturbances. The position of the second zone, its geomagnetic latitude, is controlled by the intensity of the solar magnetic field. Existing differences between the quantified results of Alfvén's theory and auroral observations were discussed by Feldstein (1986).

Hultqvist $(1958,1959)$ showed that the maximum isoauroras are adjusted along the corrected geomagnetic parallels, which take into account the difference between the real geomagnetic field and the dipole field. Precisely this causes the differences between the maximum isoauroras from the geomagnetic parallels and the different forms of the isoauroras in the Northern and Southern Hemispheres.

\section{Discrete auroras during magnetic quietness and the arc orientations}

Photographic records of auroral luminosity by means of ASCs during the IGY allowed assembling spatial distribution patterns of discrete auroral forms for definite UT moments at different levels of geomagnetic activity. Such possibilities were envisioned at the beginning of the last century by the pioneers of auroral research Kristian Birkeland and Carl Størmer. The construction of planetary auroral maps implies simultaneous observations of a large number of ASCs with intersecting ranges of vision, which was hence possible in the World Data Centers (WDCs): the WDC-A in the USA. (Akasofu, 1963) and the WDC-B in the Soviet Union (Khorosheva, 1961; Feldstein and Starkov, 1967a; Starkov and Feldstein, 1970). The auroral luminosity of the upper atmosphere proved to be a permanent phenomenon. It can be observed in the auroral oval at any level of geomagnetic activity.

Aurorae exist along a confined oval both at the dayside and nightside of Earth, even during very geomagnetically quiet days with $\Sigma K p \leq 80$ (Feldstein et al., 1966). The auroras are situated at $\Phi^{\prime} \sim 78-80^{\circ}$ CGL during daytime hours, and $\Phi^{\prime} \sim 70^{\circ} \mathrm{CGL}$ at night. A generalised pattern of discrete auroral forms in the Northern Hemisphere during such periods is presented in Fig. 5 (Feldstein et al., 1966; Feldstein, 1966). The oval is fixed with respect to the sun. Various forms of auroral luminosity are distributed along the oval in dependence of local time with a preponderance of rayed forms during daytime hours. More than one auroral form can be seen at the firmament during nighttime hours. The change of forms above stations at latitudes within the oval occurs mainly due to the motion of the observer with Earth's rotation under a fixed auroral pattern. Weak auroral substorms, however, can be generated during quiet-time periods (Akasofu et al., 1971). The local time sequence of auroral form alterations also changes during such events.

At the polar cap within the auroral oval, sun-aligned and rapidly varying auroras of low intensity appear and disappear 
even during geomagnetically quiet days. Their morphology strictly differs from the auroras in the auroral oval. This kind of aurora, drawn in Fig. 5 as waved lines, is aligned along the noon-midnight meridian and will be discussed in more detail in Sect. 5.

Feldstein et al. (1966) determined from the ascaplots the appearance frequency of auroras in the zenith both around noontime and close to midnight hours. For geomagnetically quiet conditions with $K p \leq 1+$, the maximum in the latitudinal profile of the auroral appearance frequency is located at $\Phi^{\prime} \sim 70^{\circ}$ during nighttime hours and $\Phi^{\prime} \sim 79^{\circ}$ during daytime. These latitudes of maximum appearance frequency for geomagnetically quiet conditions were observed both during the IGY (maximum of solar activity cycle) and during the IQSY (1964-1965, minimum of solar activity).

Akasofu (1964b), Stringer et al. (1965), and Feldstein (1966) discuss the position of zenith auroral forms near midnight for very quiet days. Observations from drifting (on ice) stations in the Arctic Sea, Arctic Ice Flow $\left(\Phi \sim 78.3^{\circ}\right)$ and Arctica $2\left(\Phi \sim 77.5^{\circ}\right)$ were used. The observations performed on these stations confirmed the position of the nighttime sector of the oval at $\Phi \sim 70^{\circ}$ during very geomagnetically quiet days.

Lassen (1963) and Lassen et al. (1986) presented the spatio-temporal distribution of the auroral appearance frequency for the International Quiet Days (IQD) of the IGY, based on observations of the four Greenland stations. This is reproduced in Fig. 6. It characterises the isoaurora concentrations in the morning and afternoon hours with maxima at $\Phi^{\prime} \sim 78-80^{\circ}$ CGL for 09:00-10:00 and 14:00-16:00 MLT and expanded isolines toward the midnight meridian. A discontinuity between the afternoon and nighttime isoauroras exists from 18:00 to 19:00 MLT, where the appearance of the aurora abruptly decreases.

The possible existence of such azimuthal discontinuities in the auroral occurrence within the auroral oval was mentioned by several authors. Feldstein (1963b) and Akasofu (1976) draw their attention to the difference of morphological characteristics of discrete auroral forms in the daytime and nighttime sectors of the oval:

1. Rayed forms (rays and rayed arcs) dominate in the daytime sector, while extended forms (auroral arcs and bands) prevail during the night.

2. Discrete forms move polewards or in azimuthal direction during daytime, but equatorward during nighttime.

3. There are different heights of the auroral forms: their lower boundaries are at $150-250 \mathrm{~km}$ during daytime and $100-120 \mathrm{~km}$ during the night.

4. The spectral composition of the luminosity differs between day and night with predominant red emissions during daytime and green emissions during the night.

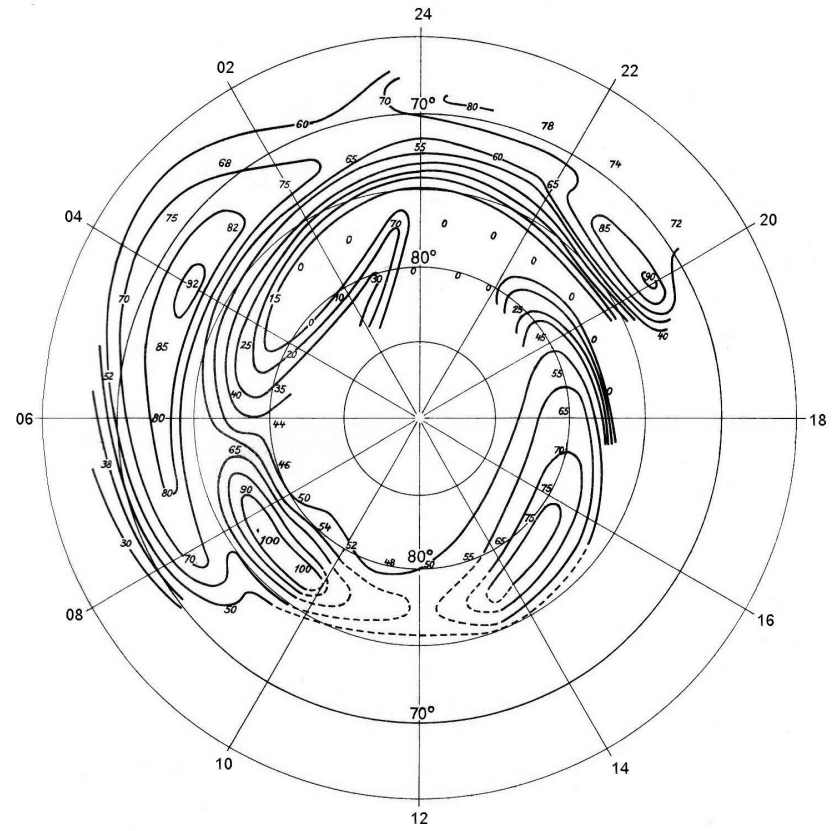

Figure 6. Auroral frequency pattern for the IQDs of 1957-1958. Isoauroral lines are drawn on the polar graph with magnetic latitude and local time (Lassen, 1963). Dashed lines illustrate interpolated isoauroral lines due to missing data during daytime hours.

Discrete auroral forms might be missing at the junctions between daytime and nighttime sectors.

Feldstein and Starkov (1967b) investigated the existence of such discontinuities in the morning and evening sectors of the auroral oval for magnetically quiet conditions in more detail. They used ascafilms of $15 \mathrm{~min}$ intervals from the stations Cape Chelyuskin $\left(\Phi^{\prime} \sim 71.2^{\circ}\right)$, Wiese $\left(\Phi^{\prime} \sim 73.4^{\circ}\right)$, and Pyramida $\left(\Phi^{\prime} \sim 74.8^{\circ}\right)$, which are located under the auroral oval in the morning and evening sectors. The aurora were most often missing from 08:00 to 10:00 MLT in the morning and during 18:00 MLT in the evening for minimum values of the geomagnetic activity. According to Meng and Lundin (1986) there is a lack of a connection between nightside substorm activity and dayside discrete auroral activity. These phenomena are consistent with the existence of two major separated injection regions along the auroral oval: the dayside cusp and the nightside plasma sheet. Discontinuities in the oval were replenished with discrete auroral forms when the disturbance level increased.

One of the peculiarities of the scheme shown in Fig. 5 is the lack of auroral arcs in the oval around noontime. Here, one observes individual rays during geomagnetically quiet periods, while there are extended arcs and bands in all other parts of the oval. The discontinuity of extended discrete auroral forms in the daytime sector was later discovered in airplane (Buchau et al., 1970) and satellite studies (Akasofu, 1976; Snyder and Akasofu, 1976; Dandakar and Pike, 1978; Meng, 1981; Murphree et al., 1982). The analysis of satellite 
images from the DMSP (Defense Meteorological Satellite Program) showed that the discontinuity has a width of about $15^{\circ}$ in longitude and occurs most often near 11:00 MLT and centred at $74.8^{\circ}$ CGL. Data of the scanning photometer on board the ISIS-2 (International Satellites for Ionospheric Studies) satellite revealed that there exists sometimes a discontinuity of the $557.7 \mathrm{~nm}$ emission on the dayside, while the $630.0 \mathrm{~nm}$ emission is continuously recorded across the noon sector.

Vorobjev and Rezhenov (1982) investigated in detail the characteristics of discontinuities in the noontime sector based on ascafilms from the Pyramida $\left(\Phi^{\prime} \sim 74.8^{\circ}\right)$ station. For the whole period of the IGY, they found only 14 intervals where a disappearance of discrete auroral forms in the daytime sector could be observed. It has been shown that in case of an existing discontinuity during low geomagnetic activity $\mathrm{AE}<100 \mathrm{nT}$, it appears in the interval of 08:30 11:30 MLT. In the region of the discontinuity one observes auroral spots and singular rays; sometimes for $2-5 \mathrm{~min}$ segments of rayed forms of very weak intensity appear, which decay to individual rays. The discontinuity occurs simultaneously with the formation of a DP-3 current system in the polar cap, which is usually observed during periods of a positive $B_{z}^{\mathbb{M F}}$ component.

Meng and Lundin (1986) performed a detailed analysis of the morphology of auroral luminosity in the daytime sector of the auroral oval. More than 300 DMSP satellite images of the years 1975 and 1979 were studied, dividing them into 5 different groups - from magnetically quiet intervals (group 1, $\mathrm{AE} \sim 50 \mathrm{nT}$, positive $B_{z}^{\mathrm{IMF}} \sim 3 \mathrm{nT}$ ) to substorm intervals (group 5, $\mathrm{AE}>500 \mathrm{nT}$, negative $B_{z}^{\mathrm{MMF}} \sim-4 \mathrm{nT}$ ). The existence of a midday gap appeared to be a permanent phenomenon, being independent of the magnetic activity level. A diffuse luminosity is observed within the gap together with an absence of discrete forms in group 1 as well as in all other groups including group 5. Auroral arcs extended along the magnetic longitude, which are typical for the auroral oval, expand into the late morning and early evening hours, adjoining the midday diffuse luminosity. The absence of discrete forms in the midday sector during magnetic disturbances according to data of Meng and Lundin (1986) might be related to the method used in this study for the identification of discrete forms. Extended auroral forms like arcs and bands are counted among discrete forms. Such forms are practically absent in the midday sector. There, one observes discrete forms as single rays, groups of rays, or fractions of rayed arcs. In addition to differences in the identification, the rayed forms in the midday sector are not sufficiently well identified with satellite observations of auroral luminosity; only these observations form the background for the statements about the permanent existence of a gap in the midday sector.

Chua et al. (1998) identified for roughly $7 \%$ of the substorms observed during their study period from December 1996 to February 1997 the appearance of nightside auroral gaps, centred around local midnight between 23:30 MLT and
00:50 MLT, spanning $1.3 \pm 0.3 \mathrm{~h}$ in local time during substorm recovery phases. By examining Polar satellite ultraviolet imager (UVI) auroral images from January 1997 to August 1998, Shue et al. (2002) found in $12 \%$ of the surveyed images two-component auroras. This two-cell convection aurora with discrete forms in the evening and morning sectors is azimuthally elongated over extended local times with a gap on the dayside and nightside. The events found were basically in the creation (growth) phase or the recovery phase of substorms. Shue et al. (2006) shows that the dusk-side branch of the two-cell aurora coincides with particle precipitations observed by the DMSP F12 spacecraft.

Holzworth and Meng (1975) and Starkov (1994) approximated the auroral oval boundaries with the first harmonics of a Fourier series. The co-latitude of the oval's equatorward boundary for magnetically quiet conditions can be described according to Starkov (1994) as

$\Theta^{\circ}=17.5^{\circ}+3.4^{\circ} \cos \left(t-18^{\circ}\right)-0.7^{\circ} \cos \left(2 t-45^{\circ}\right)$,

where $t$ is the hour angle, counting from midnight eastward. $\Theta^{\circ}$ results in 13.4 and $20.0^{\circ}$ for daytime and nighttime hours, respectively. The width of the oval during quiet conditions amounts to $\sim 2^{\circ}$, which results in poleward boundaries of $\sim 79^{\circ}$ CGL during daytime and $\sim 72^{\circ}$ CGL during nighttime. Using optical aurora observations of DMSP satellites, Meng et al. (1977) determined the radius and the centre's position of the circle, which approximates the poleward boundary of the oval. During magnetically quiet conditions, this oval radius is $14.6^{\circ}$ and its offset $6.6^{\circ}$ from the geomagnetic pole toward the nightside. This results in a boundary at $69^{\circ}$ geomagnetic latitude during the night and $82^{\circ}$ during the day.

The variation of the latitude of the auroral oval with local time entails also a variation of the arc's and band's orientations relative to the geomagnetic field. The orientation of elongated forms can be determined with fairly good accuracy by observations from a single station. This circumstance and the importance of studying the orientation of auroral arcs for the development and verification of different theories of magnetic storms and the auroras, boosted a fairly active discussion of this topic over the last two centuries. Vegard and Krogness (1920) generalised the results of optical observations during the first IPY (1882-1883). Figure 7 shows the mean direction of the arcs on a number of polar stations, referred to the geomagnetic meridian. It is obvious that the direction of the arcs is approximately perpendicular to the geomagnetic meridians. This has been confirmed by more accurate measurements with the parallactic photographs (Størmer, 1927).

A new stimulus for deeper investigations of extended auroral forms, their location and orientation, resulted from the theory of magnetic storms and auroras by Alfvén (1950). It allowed predicting the diurnal variation of the arc's orientation: a decrease of their azimuth from 18:00 to 06:00 LT, an abrupt increase by $30^{\circ}$ at 06:00 LT, and a subsequent slight decline till 18:00 LT. The existing fragmentary observations 


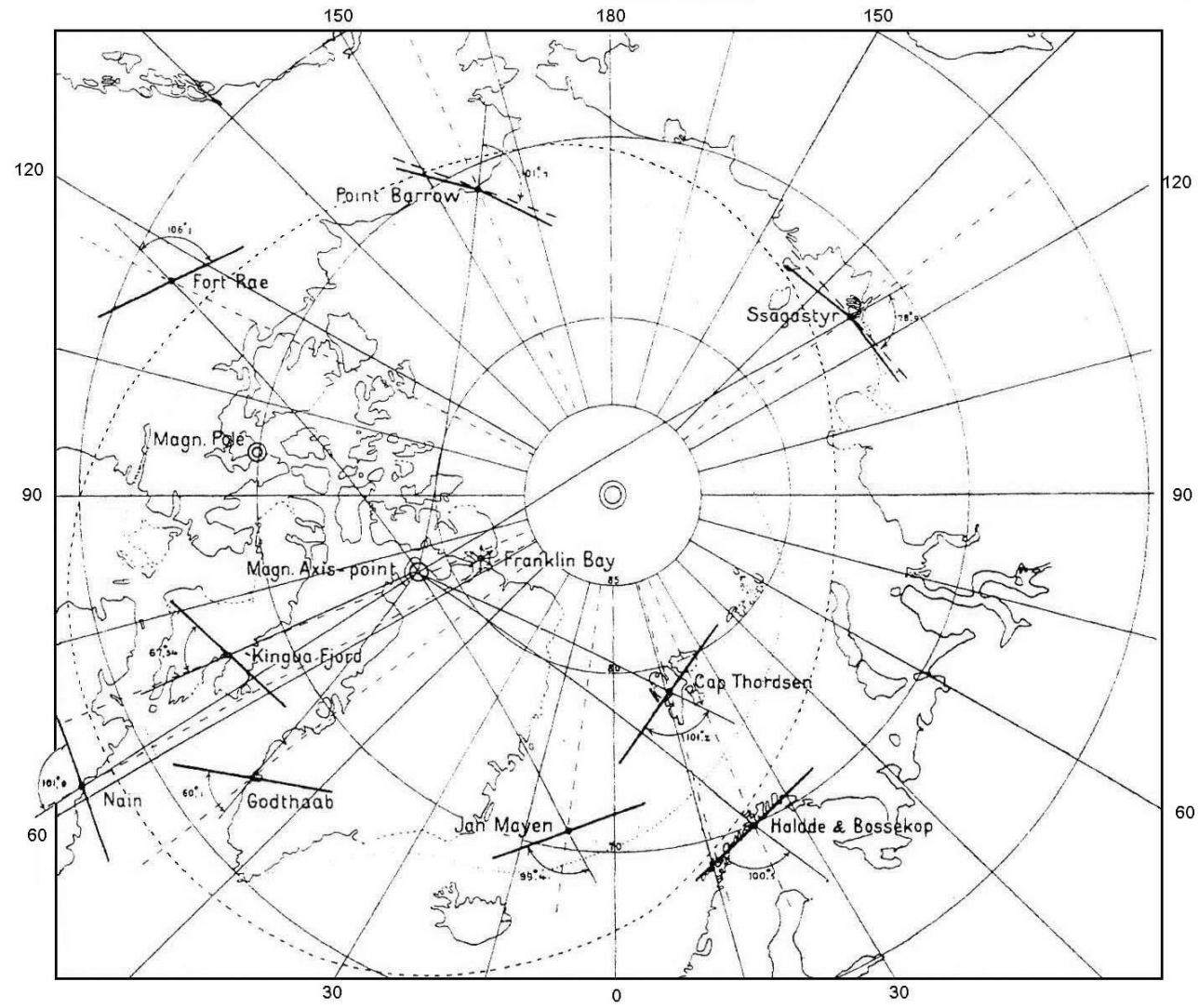

Figure 7. Mean direction of auroral arcs along the auroral zone referred to the geomagnetic meridians (Vegard and Krogness, 1920).

of the diurnal variation required further, more detailed reprocessing of the observations to make a definite check of the theory. In particular, observations at the discontinuity near 06:00 LT are very important (Alfvén, 1950, p. 188).

Such investigations were practically simultaneously performed at the stations Godhavn $\left(\Phi^{\prime} \sim 77.6^{\circ}\right)$ (Lassen, 1959), Kiruna $\left(\Phi^{\prime} \sim 64.5^{\circ}\right)$ (Hultqvist et al., 1961), and Dixon Island $\left(\Phi^{\prime} \sim 67.9^{\circ}\right)$ (Starkov and Feldstein, 1960). The attention was focused particularly on the existence of an azimuthal jump in the morning hours. Such a jump was found in the observations of Godhavn and Kiruna in full correspondence to Alfvén's theory. The observations on Dixon Island showed a systematic decrease of the azimuth from 18:00 to 06:00 LT, qualitatively supporting the theory. The existence of an azimuthal jump in the morning hours could not be verified, because around 06:00 LT at these latitudes diffuse auroras were predominant, and the discrete forms were shifted poleward away from Dixon Island. To obtain a somehow comparable impression about the azimuthal variation in the morning hours, it was necessary to involve observations from stations at higher latitudes, where the oval shifts at 06:00 LT during its latitudinal variations. Starkov and Feldstein (1967a) analysed observations at the high-latitude stations Cape Chelyuskin and Pyramida (Fig. 8a, b), which allowed following the azimuth variations of elongated auroral forms around the clock.

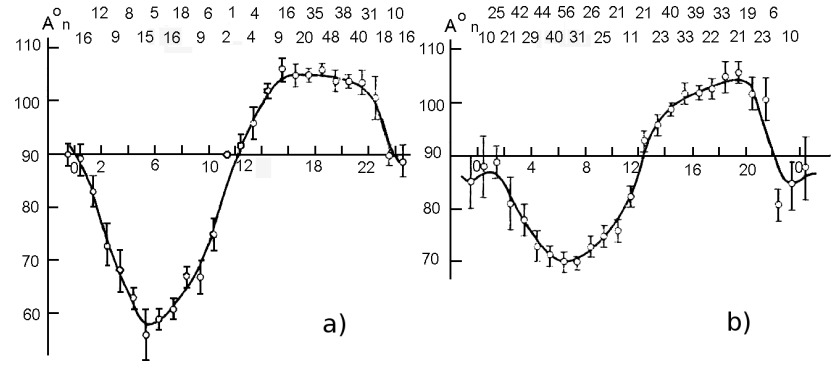

Figure 8. Diurnal variations (MLT) of the azimuths of elongated auroral forms at the stations Cape Chelyuskin (a) and Pyramida, Spitzbergen (b). The number signifies the quantity of arcs occurring during the corresponding hour. The vertical lines indicate the mean quadratic errors (Starkov and Feldstein, 1967a).

Figure $8 \mathrm{a}$ and $\mathrm{b}$ show azimuths of the arcs of $\sim 90^{\circ}$ at midday and at midnight, but there is no jump of the azimuth around 06:00 LT. In the morning hours, a rapid increase takes place, which was interpreted in previous investigations as a jump.

Assuming that the arcs are aligned along the auroral oval, which represents at a given moment the distribution of the auroral luminosity, one can deduce the position of the auroral oval from the auroral orientation. Such a method with single-station observations was proposed by Alfvén (1950) 


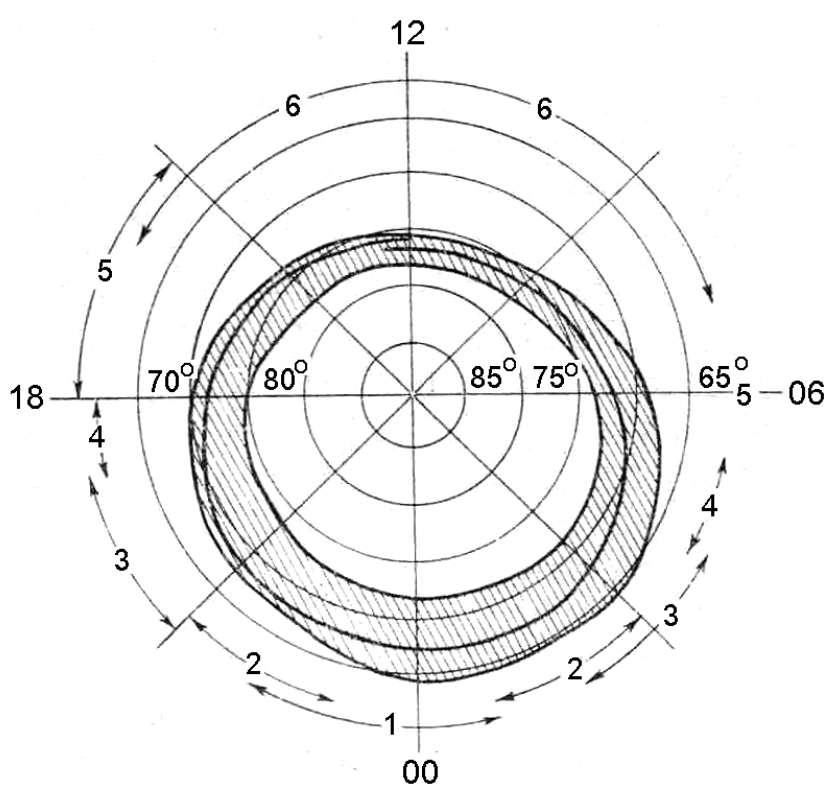

Figure 9. Auroral belt (bold line) obtained from auroral orientation observations at the stations Dixon Island (1), Point Barrow (2), Fort Churchill (3), Cape Chelyuskin (4), Wiese Island (5), and Pyramida, Spitzbergen (6). The hatched area shows the position of the auroral oval. The digits 1-6 denote the MLT intervals, during which the observed azimuths at the corresponding stations were obtained for the construction of the current auroral belt (Starkov and Feldstein, 1967a).

and performed for the first time by Hultqvist et al. (1961). Observations of the diurnal azimuthal variation of the arcs at several different latitudes, allowed an even more precise determination of the auroral band position. Figure 9 shows the results of such calculations for the IGY period according to Starkov and Feldstein (1967a). The dashed line indicates the position of the auroral oval, deduced from observations of the appearance frequency of auroras. It is obvious, that the auroral band, which is calculated corresponding to the azimuth observations, coincides with the auroral oval. An analogous result was achieved for the IQSY period by Gustafsson et al. (1969).

The practically uninterrupted appearance of bright auroral forms along the oval during any level of geomagnetic activity suggests a continuous particle flow into the upper atmosphere. Apparently, a sufficiently effective generation process for such particles within the magnetosphere is necessary. Such a process must act continuously, even during periods of low magnetic activity, and with a particle flow of the order of $10^{8}-10^{9}$ electrons $\mathrm{cm}^{-2} \mathrm{~s}^{-1}$ with energies between 1 and $10 \mathrm{keV}$.

\section{Discrete auroras in the polar cap}

Auroral arcs at latitudes poleward of the auroral oval (above $\sim 80^{\circ} \mathrm{CGL}$ ) have been systematically studied and classified as a particular class of arcs about 50 years ago based on the material of the IGY (e.g. Davis, 1960; Feldstein, 1960; Denholm and Bond, 1961). At images taken with ground-based all-sky cameras or from satellites, these auroras looked like arcs, which are elongated approximately along the noonmidnight meridian. Therefore, they have been often called sun-aligned arcs. The first person who carefully recorded such auroral forms was the Australian scientist Mawson (1916). Nowadays, also other terms like polar cap auroras (PCA), transpolar arcs, and $\theta$ auroras are used to denote sun-aligned arcs. This commonly used nomenclature mainly reflects morphological characteristics of the luminosity, but the term PCA comprises also a supposition about the likely source region of this luminosity in the magnetosphere. Indeed, it is often assumed that the region inside the auroral oval boundary is the polar cap, i.e. a region of "open" magnetic field lines and part of the magnetospheric tail. The source region of high-latitude sun-aligned arcs is debated in detail in the current literature (Sandholt et al., 2002a; Newell et al., 2009). In this review article we will utilise the term PCA, because it is the most commonly used.

Hultqvist (1962) and Feldstein (1963b) generalised the individual observational results of many high-latitude stations in the Northern and Southern Hemispheres. They showed that the orientation of the PCA follows at all stations in its diurnal variation the sun's position, but at local midnight the PCAs are oriented in the direction toward the geomagnetic pole.

The diurnal variation of the PCA appearance frequency for Northern Hemisphere stations during the IGY was investigated by Davis (1962) and later by Lassen (1972) and Ismail et al. (1977). It was shown that the PCA appearance has two maxima in the diurnal variation - one in the morning sector, the other in the evening sector of the polar cap, in which the morning maximum is larger than the evening one. Figure 10 shows the diurnal variation of the PCA appearance frequency, taken from the work of Yahnin and Sergeev (1981). Data for Northern Hemisphere stations (Fig. 10a, b, c) were adopted from the paper of Davis (1962), but show the dependence converted to MLT instead of LT. For the Southern Hemisphere, data of the Vostok station $\left(\Phi^{\prime}=-83.5^{\circ}\right)$ were used for 1965 (d) and 1968 (e). The maxima at the Alert station $\left(\Phi^{\prime}=-86.7^{\circ}\right.$, Fig. 10b) differ from the expected values, probably due to the small number of observations during evening hours. Two maxima are observed at the Vostok station, where the morning maximum is larger than the evening maximum.

The orientation of the interplanetary magnetic field (IMF) has a substantial influence on the appearance and distribution of PCAs. Hourly averages of the $B_{z}^{\mathrm{MF}}$ component during PCA observations with satellites (Berkey et al., 1976) and ASCs (Lassen and Danielsen, 1978) turned out to be mostly positive. The appearance of sun-aligned arcs in the polar cap area as a predominantly northward $B_{z}^{\mathrm{IMF}}$ phenomenon was described also by Yahnin and Sergeev (1979), Gussenhoven 

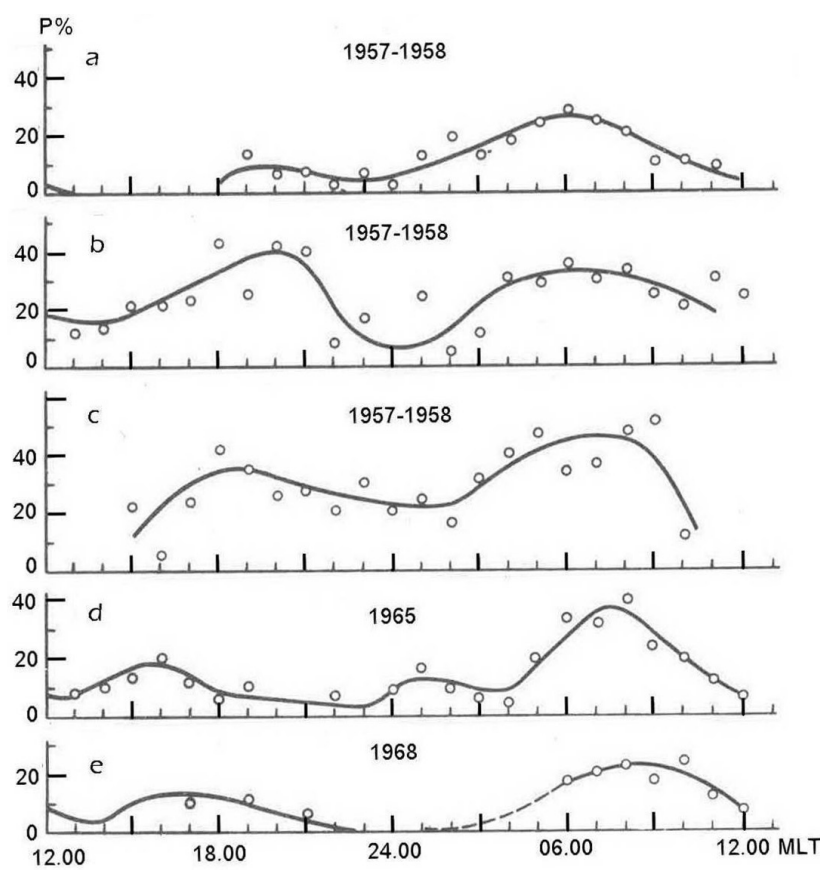

Figure 10. Diurnal variation of the PCA appearance frequency for the stations Thule (a), Alert (b), and Resolute Bay (c) in the Northern Hemisphere and the station Vostok $(\mathbf{d}, \mathbf{e})$ in the Southern Hemisphere (after Yahnin and Sergeev, 1981).

(1982), Valladares et al. (1994), Cumnock et al. (1997) and others. Troshichev et al. (1988) argued that arcs appear in the polar cap with a time delay of $\sim 1 \mathrm{~h}$ relative to a northward turning of the IMF, while its disappearance after a southward turning of the IMF occurs much faster, usually within 10$15 \mathrm{~min}$. Rodrigues et al. (1992) determined 20-30 min as the characteristic time of PCA decay.

The effects of the $B_{y}^{\mathrm{IMF}}$ and $B_{x}^{\mathrm{IMF}}$ components' signs on the PCA behaviour were discussed in numerous papers (e.g. Lassen and Danielsen, 1978; Yahnin and Sergeev, 1979, 1981; Gussenhoven, 1982; Troshichev and Gusev, 1994). Lassen and Danielsen (1978) showed with data from stations in Greenland that in the Northern Hemisphere PCAs occur more often for $B_{y}^{\mathrm{IMF}}>0$ and $B_{x}^{\mathrm{IMF}}<0$. Using ascaplots of high-altitude stations of the Northern (Nord, $\Phi^{\prime}=80.7^{\circ}$; and Thule, $\Phi^{\prime}=86.1^{\circ}$ ) and of the Southern Hemispheres (Scott Base, $\Phi^{\prime}=80.1^{\circ}$; Wilkes, $\Phi^{\prime}=80.7^{\circ}$; Dumont d'Urville, $\Phi^{\prime}=80.4^{\circ}$; and Vostok, $\left.\Phi^{\prime}=83.5^{\circ}\right)$, Yahnin and Sergeev (1979) discovered an asymmetric probability of auroral appearances in the opposite polar caps. According to them, PCAs at high latitudes of the Southern Hemisphere are more often observed for $B_{y}^{\mathrm{MFF}}<0$ and $B_{x}^{\mathrm{MF}}>0$, while in the Northern Hemisphere more often for $B_{y}^{\mathrm{IMF}}>0$ and $B_{x}^{\mathrm{IMF}}<0$.

Figure 11a illustrates the PCA appearance frequency in the Southern Hemisphere in dependence of the $B_{z}^{\mathrm{IMF}}$ component (Yahnin and Sergeev, 1981). The $B_{z}^{\mathrm{IMF}}$ values are sampled in steps of $1 \mathrm{nT}$ and confined to the range of
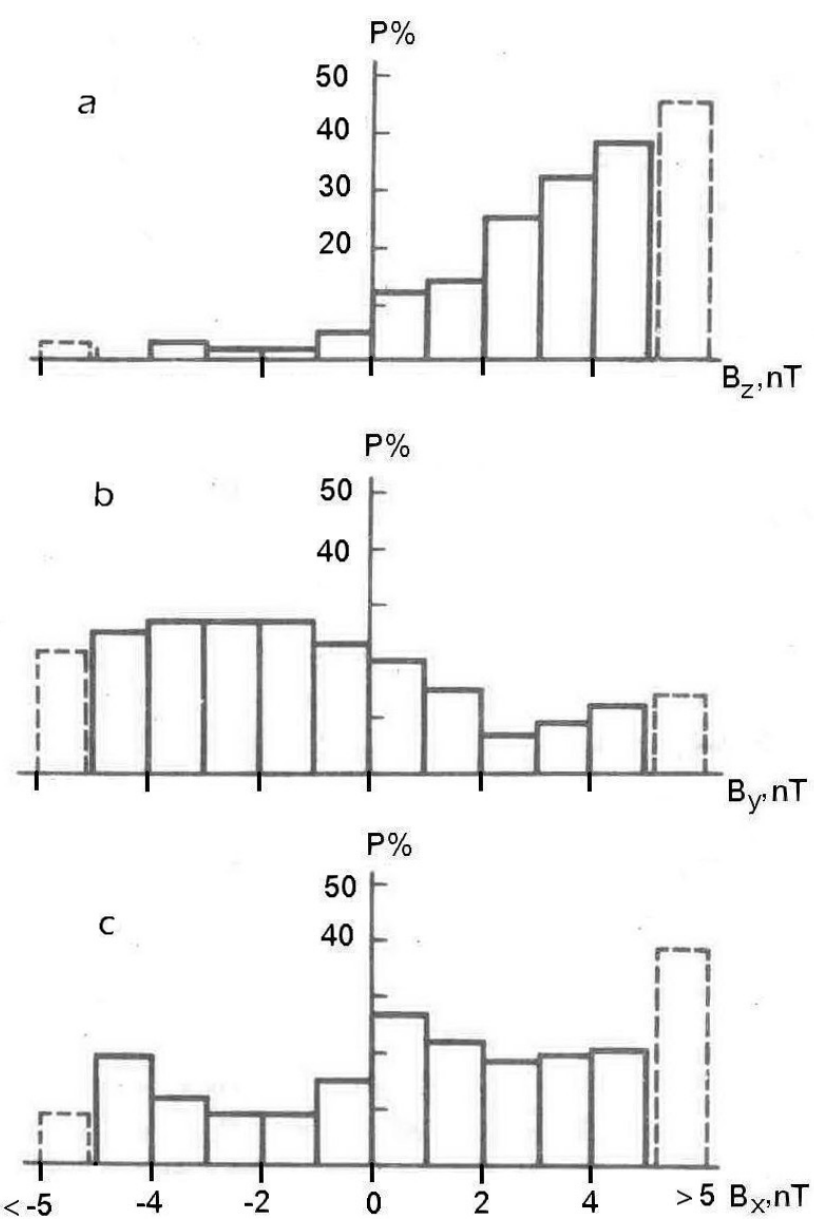

Figure 11. Dependence of the PCA appearance frequency in the Southern Hemisphere on the magnitude and polarity of the $B_{z}^{\mathrm{IMF}}$ component (a) as well as on the $B_{y}^{\mathrm{IMF}}$ (b) and $B_{x}^{\mathrm{MF}}$ (c) components for positive $B_{z}^{\mathrm{IMF}}\left(B_{z}>0\right)$ values (Yahnin and Sergeev, 1981).

$-6 \mathrm{nT} \leq B_{z}^{\mathrm{IMF}} \leq+6 \mathrm{nT}$. The number of aurora observations was insufficient for $B_{z}^{\mathrm{IMF}}<-5 \mathrm{nT}$ and $B_{z}^{\mathrm{MF}}>+5 \mathrm{nT}$; the outer columns (dashed) show the sum of them. The PCA appearance frequency for southward IMF (negative $B_{z}^{\mathrm{IMF}}$ ) is quite small, while it increases linearly for the northward IMF orientation (positive $B_{z}^{\mathbb{M F}}$ ). Observations of auroras in the southern polar cap for northward $B_{z}^{\mathrm{IMF}}$ are more often for $B_{y}^{\mathrm{MF}}<0$ and $B_{x}^{\mathrm{IMF}}>0$ (Fig. 11b, c). Sandholt et al. (1993) considered the direction of motion of discrete auroral forms at the dayside boundary of the polar cap for both positive and negative $B_{y}^{\mathrm{IMF}}$ component values.

Due to the spiral structure of the IMF, there is a strong correlation between the $B_{x}^{\mathrm{IMF}}$ and $B_{y}^{\mathrm{IMF}}$ components. Yahnin and Sergeev (1981) considered, therefore, the diurnal variation of the PCA appearances during periods when the IMF vector resided in different quadrants of the GSM $X-Y$ plane, to separate the effects of these components. These investigations revealed that the appearance frequency in the Southern 
Hemisphere increases for $B_{x}^{\mathrm{IMF}}>0$ independently of the $B_{y}^{\mathrm{IMF}}$ sign (cf. Fig. 11a-c).

The importance of the $B_{y}^{\mathrm{IMF}}$ component is particularly obvious in the appearance frequency of auroras in the dawn and dusk sectors of the polar cap. Sun-aligned arcs are observed in the dawn (dusk) side of the northern polar cap much more frequently during $B_{y}^{\mathrm{MMF}}>0\left(B_{y}<0\right)$ (Yahnin and Sergeev, 1979; Gussenhoven, 1982). On the contrary, auroras appear in the dawn (dusk) side of the southern polar cap most frequently during $B_{y}^{\mathrm{IMF}}<0\left(B_{y}^{\mathrm{MPF}}>0\right)$ (Yahnin and Sergeev, 1981; Troshichev et al., 1988).

Sergeev and Yahnin (1978) selected 117 cases of moving arcs from the Northern Hemisphere and 82 cases from the Southern Hemisphere to investigate the direction of PCA motions in more detail. They showed that in both hemispheres motion toward the dusk (dawn) side dominates in the dawn (dusk) sector, respectively, i.e. the movement always occurs from lower to more poleward latitudes. Data analyses from former studies (Feldstein et al., 1968; Danielsen, 1969; Akasofu, 1972a) showed a good correspondence with this result, considering the local time dependence of these observations.

Because PCAs occur mainly for northward $B_{z}^{\mathbb{M F}}$, their appearance probability is hence obviously anticorrelated to the geomagnetic disturbance level and to the activity of substorms (Davis, 1963; Feldstein et al., 1969; Starkov and Feldstein, 1971a; Lassen, 1972; Gussenhoven, 1982; Ismail et al., 1977). Starkov and Feldstein (1971a) proposed to include PCAs into a substorm development scheme. In this scheme, polar cap auroras disappear during the transition from the creation phase to the expansive phase of a substorm. This rule generally holds, but there are reports about a number of events where PCAs were observed during substorm expansion phases (e.g. Pike and Whalen, 1974; Akasofu, 1974a; Yahnin and Sergeev, 1981; Henderson et al., 1996). Prior to the expansion phase, the IMF is usually, but not always, southward oriented $\left(B_{z}^{\mathrm{IMF}}<0\right)$, obviously causing the disappearance of auroras from the polar cap (for southward $B_{z}^{\mathrm{MF}}$ ) or its existence simultaneously with a developed auroral bulge (for northward $B_{z}^{\mathrm{IMF}}$ ).

Frank et al. $(1982,1986)$ described a particular class of PCAs, the $\theta$ auroras, which were observed with the UVI imager on board the Dynamics Explorer 1 (DE-1) spacecraft. The $\theta$ aurora is a transpolar, sun-aligned band linking dayside and nightside parts of the auroral oval. The relation of the $\theta$ aurora to the $B_{y}^{\mathrm{IMF}}$ component was examined by Frank et al. (1985). They showed that the motion of the transpolar band is generally in the direction of the $B_{y}^{\mathrm{IMF}}$ component in the Northern Hemisphere and in the opposite direction in the Southern Hemisphere. Makita et al. (1991) argued that $\theta$ auroras are usually located at the poleward edge of the soft, $E<0.5 \mathrm{keV}$, precipitation region extending from the morning (evening) parts of the auroral oval during $B_{y}^{\mathbb{M F}}<0$ $\left(B_{y}^{\mathrm{IMF}}>0\right)$. Distributions of $\theta$ auroras for different IMF ori- entations were also obtained from Viking spacecraft UVI images by Elphinstone et al. (1990).

The scanty spatial resolution of spacecraft UV imagers did not allow examining the fine structure of $\theta$ auroras and their relation to visible PCAs. However, there were some reasons to assume that visible PCAs are the most intense brightenings, appearing against the background of wider but less luminous transpolar bands that were observed by UV imagers (Gusev and Troshichev, 1990). Direct comparisons of visible PCAs observed by the all-sky camera at the Vostok station and $\theta$ auroras registered by the DE-1 UV imager were carried out for the first time by Vorobjev et al. (1995b). They examined the event of 3 August 1986, when sun-aligned arcs persisted in the sky over the Vostok station for an unusually extended period of about $5 \mathrm{~h}$. The $B_{z}^{\mathrm{IMF}}$ and $B_{y}^{\mathrm{IMF}}$ components observed with the IMP-8 spacecraft from 14:00 UT to 20:00 UT are shown in the two left-hand side panels of Fig. 12. In this case, the propagation time from the IMP-8 location to the magnetopause was $\sim 7 \mathrm{~min}$. The auroral dynamics obtained from ground-based all-sky cameras are represented in the right-hand side panel of Fig. 12. The magnetic local time at Vostok station (MLT $=\mathrm{UT}-1$ ) is shown along the right vertical axis. The horizontal axis shows the distance $(\mathrm{L}, \mathrm{km})$ from the noon-midnight meridian under the assumption that the aurora height was about $150 \mathrm{~km}$. The auroral display was reversed left to right to provide a view from the Northern Hemisphere with the noon toward the top and local evening toward the left. The region occupied by the PCA and the $\theta$ aurora is shown as a hatched band.

Observations with the DE-1 spacecraft in the dark Southern Hemisphere and with Viking spacecraft in the sunlit Northern Hemisphere, which were available for this event, showed that the auroras have a typical $\theta$ structure. Figure 13a illustrates the boundaries of the UVI auroral luminosity observed by DE-1 with a brightness of $1 \mathrm{kR}$ at 18:24 UT (Craven et al., 1991). The hatched region of the auroral luminosity is shown in coordinates of corrected geomagnetic latitudes (CGL) versus MLT. The field of view of Vostok's all-sky camera and the discrete auroral arcs recorded at 18:24 UT are superposed on the UVI auroral distribution pattern. The camera field of view was confined to $500 \mathrm{~km}$. The original all-sky camera frame at 18:24 UT is shown in Fig. 13b. It presents three rather bright sun-aligned arcs embedded in the transpolar band of the UV $\theta$ aurora.

Craven et al. (1991) presented the position of the transpolar band boundaries in the dawn-dusk meridian (06:00-18:00 MLT axes) from 17:48 UT to 19:48 UT. These contours of $1 \mathrm{kR}$ brightness are shown with crosses connected by thin lines in the right part of Fig. 12. As can be seen in the figure, the visible sun aligned arcs are located inside the transpolar band. The horizontal dashed line indicates here the time of 18:24 UT. Auroral distributions observed simultaneously with DE-1 and Viking spacecrafts in both hemispheres are represented in Fig. 13c. The boundaries of auroral luminosity observed with DE-1 at 18:24 UT are shown 


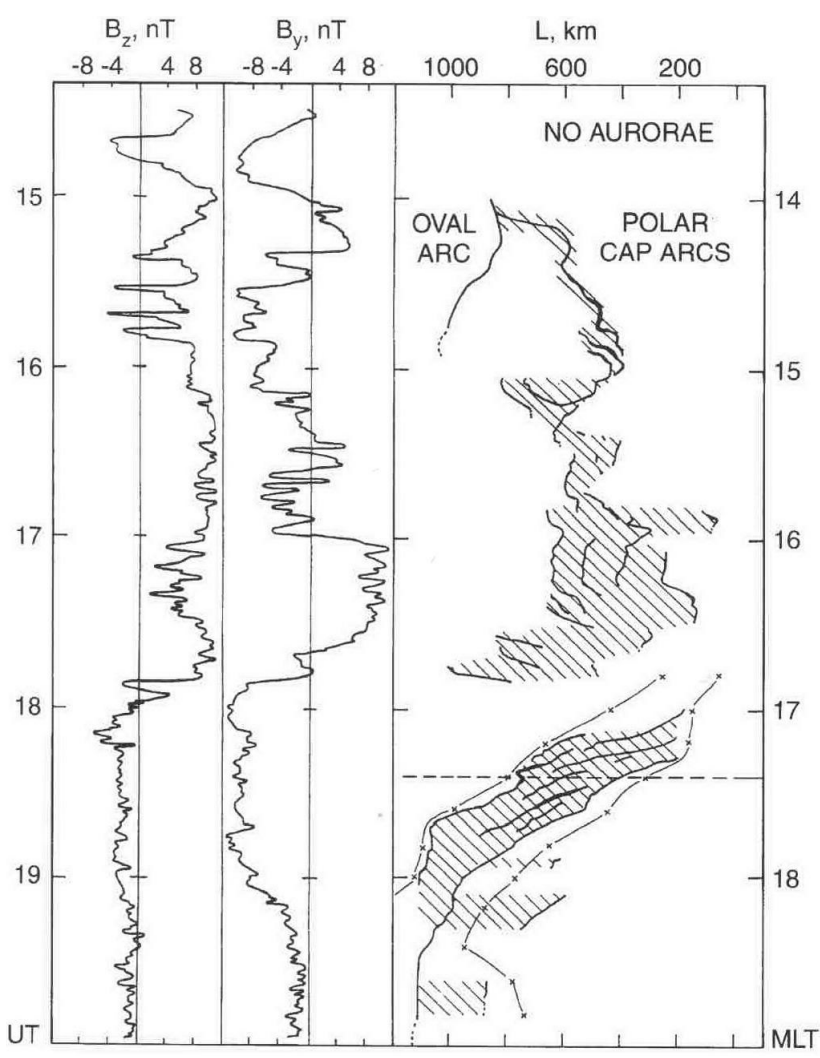

Figure 12. Variations of the $B_{z}^{\mathrm{IMF}}$ and $B_{y}^{\mathrm{IMF}}$ components obtained with the IMP-8 spacecraft and the auroral dynamics in the sky of the Vostok observatory $\left(\Phi^{\prime}=83.5^{\circ}\right)$ on 3 August 1986 (Vorobjev et al., 1995a).

by solid contours. The dashed line indicates the approximate centre of the transpolar band. The position of northern auroras observed simultaneously with Viking at 18:25 UT is superposed on the southern auroral display. The thick line marks the position of the northern transpolar band.

The intensity of auroral luminosity recorded by Viking was divided into four gradations in arbitrary units. Craven et al. (1991) noted that the transpolar bands in both hemispheres are located almost symmetrically relative to the 13:00-01:00 MLT meridian. In addition to optical observations, Feldstein et al. (1995) used auroral plasma distribution records from the Viking and DMSP F6 and F7 spacecrafts of 3 August 1986, and showed that the conclusion of Craven et al. (1991) about the asymmetrical location of the $\theta$ aurora in both hemispheres is applicable only to the most intense luminosity sites. Precipitation data support the location of the most intense aurora in the Northern Hemisphere as being in the morning sector but show that a less intense arc occurs in the evening sector, in a conjugate position to the $\theta$ aurora observed in the Southern Hemisphere. Thus, the precipitation pattern, but not the intensity of luminosity, is conjugate. The asymmetry in the polar cap auroral intensity is probably controlled by the $B_{y}^{\mathrm{IMF}}$ component direction. The event
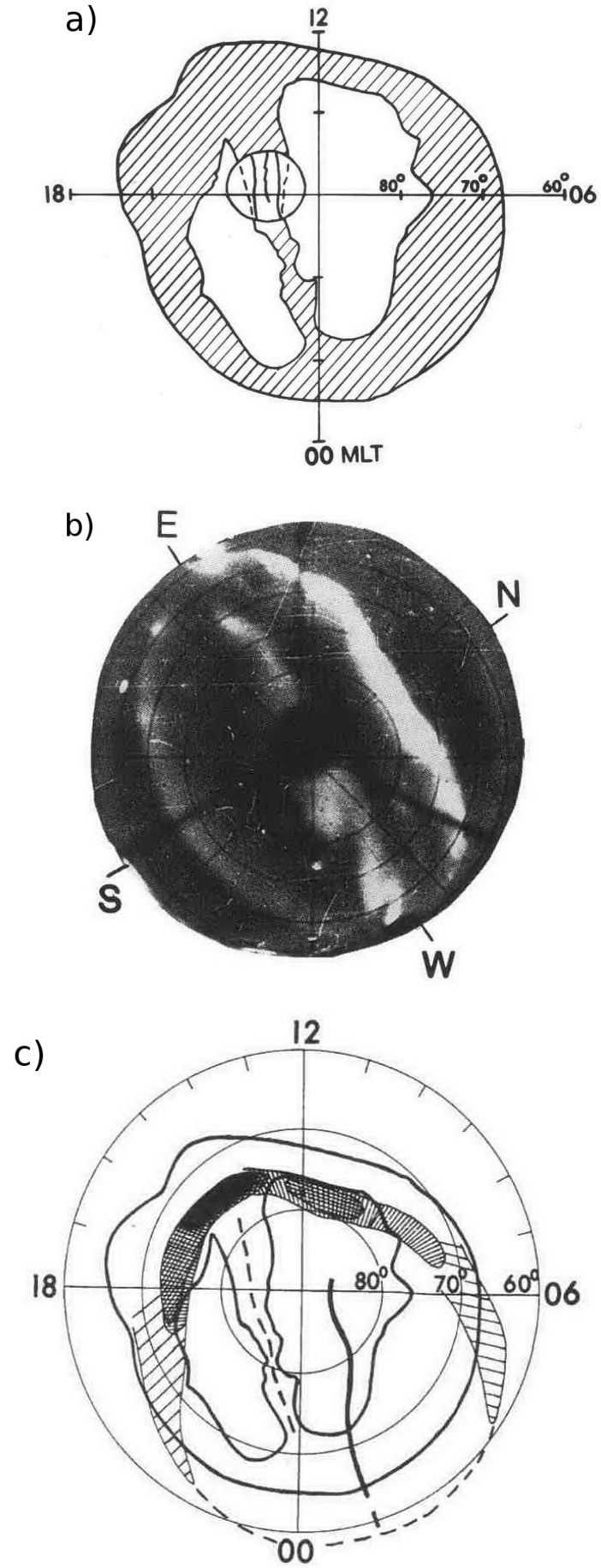

Figure 13. Distribution of the auroral luminosity over the Vostok observatory in the Southern Hemisphere $\left(\Phi^{\prime}=-83.3^{\circ}, \Lambda^{\prime}=\right.$ $53.8^{\circ}$ ) on 3 August 1986, 18:24 UT: (a) boundaries of UVI auroral luminosity in the Southern Hemisphere, the dashed area indicates the auroral luminosity, (b) the frame of all-sky camera film from the Vostok observatory, and (c) simultaneous aurora distributions in both hemispheres (different shadings indicate different auroral intensities) (Vorobjev et al., 1995a). 
under discussion occurred, when the IMF components were $B_{z}=-2.5 \mathrm{nT}, B_{y}=-9.5 \mathrm{nT}$, and $B_{x}=5.5 \mathrm{nT}$. Let us note that this $\theta$ aurora persisted for a long time during a southward IMF orientation. Unquestionably, most isolated $\theta$ auroras across the polar cap occur for northward IMF (Cumnock et al., 1997).

Zverev et al. (1992) provided a compilation of simultaneous observations of discrete auroral forms in the northern (with the Viking UVI imager) and southern polar cap regions (all-sky cameras at the Vostok station) for various situations. It was shown that PCAs in opposite hemispheres can

1. appear in the same time sector

2. be distributed approximately symmetrical to the noonmidnight meridian, or

3. be observed only in one hemisphere.

These results, as well as the asymmetry of the auroral appearance frequency in opposite hemispheres in dependence on the $B_{y}^{\mathrm{IMF}}$ component, can provide evidences about the violation of auroral conjugacy, which would be expected for arc generations at closed magnetic field lines. All PCA events examined by Zverev et al. (1992) as well as the event of 3 August 1986, presented above, were observed during southward $B_{z}^{\mathrm{IMF}}$ conditions. Therefore, one can tell only about a statistical dependence of PCA appearances on the $B_{z}^{\mathrm{IMF}}$ orientation.

In the paper of Vorobjev and Zverev (1995), some morphological features of PCAs were examined, based on fifteen events including the one on 3 August 1986, by using all-sky camera films from the Vostok station. Abruptly rising sunaligned arcs of 15-30 min duration were observed, during which the auroral brightness increased by a factor of 5-10. These auroral activations occurred both for northward and southward oriented IMF, with a tendency for longer lasting events if $B_{z}^{\mathrm{MFF}}$ is southward. Vorobjev and Zverev (1995) reported at the 22nd European Meeting on Atmospheric Studies by Optical Methods that such polar cap aurora events (PCE) under southward $B_{z}^{\mathrm{IMF}}$ conditions last about two times longer than those under northward $B_{z}^{\mathrm{IMF}}$. They argued that auroral displays during PCEs often resemble midnight auroral substorm developments for positive or small southward $B_{z}^{\mathrm{IMF}}$. The only difference is that PCEs were not accompanied by an increase of ionospheric currents. Later, Kornilov et al. (2008) examined sun-aligned arc features from all-sky TV (televison) camera observations in Barentsburg, Spitzbergen $\left(\Phi^{\prime}=75.2^{\circ}\right)$. They found out that during northward $B_{z}^{\mathrm{MPF}}$ the arc brightness increased 10-20-fold on a timescale of 5$10 \mathrm{~min}$. At the peak of brightening, polar cap arcs exhibited fast motions and deformations. They concluded as well that polar arc activations look like an auroral zone breakup, but rotated by $90^{\circ}$.

Newell et al. (2009) found three types of polar cap auroras when investigating the characteristics of auroral precipitations with the DMSP satellites. The most common type constitutes a weak intensification of the polar rain (the continuous corpuscular precipitation within the polar cap), with energy fluxes $F<0.1 \mathrm{erg} \mathrm{cm}^{-2} \mathrm{~s}^{-1}$ for electrons and an absence of corresponding ion fluxes. The second type consists according to the authors of electron and ion fluxes, which are adjacent to the auroral oval with flux intensities of $F>0.1 \mathrm{erg} \mathrm{cm}^{-2} \mathrm{~s}^{-1}$. Finally, intense electron and ion precipitations, which are well detached from the auroral oval, constitute the third type. They contain plasma sheet ion precipitations as well as electrons.

The electrodynamics of auroras in the polar cap and their relation to the characteristics of auroral precipitations, to the distribution of electric fields and currents within the polar cap and to the plasma structure of the magnetosphere, are described in the review articles of Sandholt and Farrugia (2002), Newell et al. (2009), and in the monograph Auroral Plasma Physics (Paschmann et al., 2002).

\section{Auroral luminosity in the daytime sector}

Investigations of the spectral and morphological characteristics of daytime aurora constitute matters of particular interest. It was shown already in the very first studies of this kind (Fairfield, 1968) that during daytime hours the projection of the auroral oval along the geomagnetic field lines agrees very well with the magnetospheric boundary, the magnetopause. The main energy transfer processes from the magnetosheath region into the magnetosphere-ionosphere system take place at the magnetopause and in the magnetospheric boundary layers.

The auroral activity in the oval latitudes exists almost continuously. Ground-based observations during the IGY showed the presence of discrete forms in the daytime and nighttime hours even in extremely magnetically quiet periods (Fig. 5). An analysis of ascafilms during such intervals has shown that the daytime sector of the auroral oval is filled up by separated auroral rays and then by faint rayed arcs along with a magnetic activity increase (Feldstein and Starkov, 1967a; Starkov and Feldstein, 1967b).

During magnetic disturbances the equatorward boundary of the oval shifts to lower latitudes at all MLT hours whereas the behaviour of its poleward boundary is more complicated. The nighttime sector of the oval expands and the area covered by auroras increases. The dependence of the Northern Hemisphere auroral oval position in the daytime 06:00-10:00 MLT (a), 10:00-14:00 MLT (b) and 14:00-18:00 MLT (c) sectors on the magnetic activity level, expressed by the $Q$ index, is shown in Fig. 14 (Feldstein and Starkov, 1967a). As seen in Fig. 14b, the midday part of the oval shifts equatorward almost without expansion. The centre of midday auroras displaces toward the south from about 77 to $74^{\circ}$ CGL, when the $Q$ index increases from 0 to 6 .

The nighttime aurora dynamics is defined mainly by the auroral substorm development while midday auroras are less 

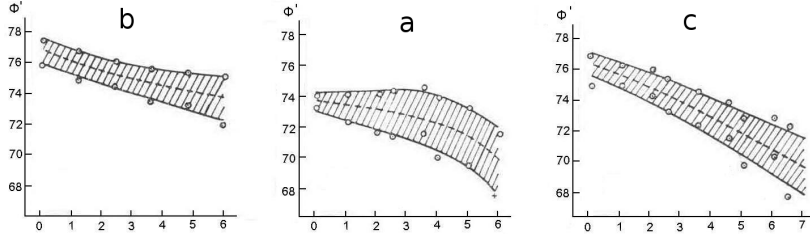

Figure 14. Locations of the northern and southern edges of the dayside auroral oval in dependence on the $Q$ index of the magnetic activity (a 06:00-10:00 MLT, (b) 10:00-14:00 MLT, and (c) 14:00-18:00 MLT). The dashed line represents the centre of the oval (Feldstein and Starkov, 1967a).

expressively included in the substorm activation. The original version of the auroral substorm development scheme (Akasofu, 1964a) was completed to a global pattern by including the dynamics of daytime auroras by Feldstein and Starkov (1967a), Starkov and Feldstein (1967b), and then by Akasofu (1968). A new feature of this pattern was the equatorward displacement of the midday auroras during the substorm expansive phase. This equatorward displacement has been confirmed later by Akasofu (1972b). Akasofu (1972c) has found a close relationship between the range of the equatorward shift of midday aurorae and the magnitude of a negative magnetic bay in the midnight sector of the auroral zone.

In the papers cited above, the displacement of midday auroras was considered as a change of whole auroral zone position while the dynamics of each discrete auroral form was not investigated in detail. Such kind of study was carried out for the first time by Vorobjev et al. (1975), and then in more detail by Vorobjev et al. (1976b). With numerous events it was shown that, inside the auroral oval boundaries, the dayside rayed auroral forms move predominantly poleward, just like prior to magnetospheric substorms and when substorms are in the progress. These authors used $1 \mathrm{~min}$ ascafilms obtained from the Pyramida station (Spitzbergen) during the IGY period to estimate the dayside arc's velocity, which can reach $0.8 \mathrm{~km} \mathrm{~s}^{-1}$. The average poleward velocity was calculated to be about 230 and $330 \mathrm{~m} \mathrm{~s}^{-1}$ before and during the substorm expansive phase, correspondingly. To estimate the auroral velocity, the altitude of discrete auroras was adopted to be $150 \mathrm{~km}$ (Starkov, 1968).

The dayside auroral luminosity is characterised by a fairly complex structure; the oval with discrete forms is only a part of the dayside auroras (Heikkila et al., 1972; Shepherd et al., 1976; Vorobjev and Turjansky, 1983; Sandholt et al., 1985; Vorobjev et al., 1988a). Dayside poleward moving auroral forms are observed inside a broad band of red auroral luminosity. They generally occur near its equatorward edge and disappear inside of this area, as a rule, not reaching its polar boundary. The red auroral band is therefore about $1-2^{\circ}$ wider than the oval of discrete forms. Separated rays and faint, longitudinally bordered rayed arcs can appear sometimes in this part of red luminosity for a short time.

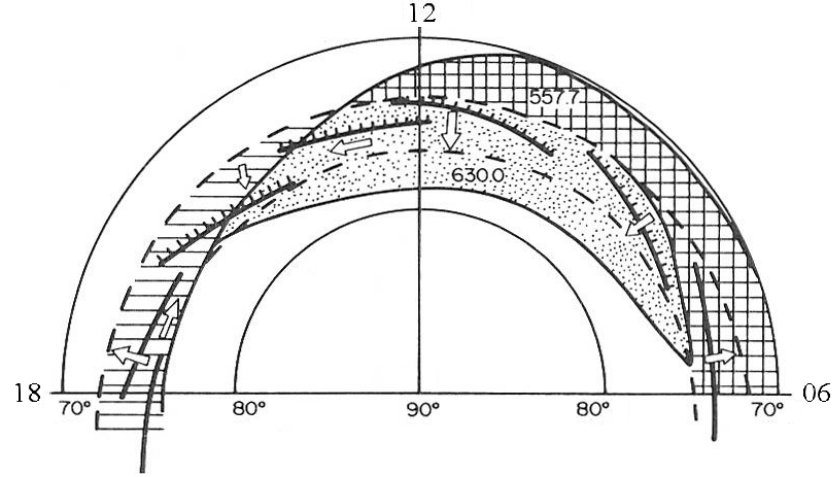

Figure 15. Distribution of the dayside auroral luminosity during a mean level of magnetic activity. Boundaries of the auroral oval are shown by dashed lines. The position of homogeneous arcs is shown by solid lines; that of rayed ones by a herringbone. The direction to the sun is upward in the figure (Leontyev et al., 1992).

The dayside red auroral band is the region where the auroral luminosity in the (OI) $630.0 \mathrm{~nm}$ emission prevails over the $\left(\mathrm{N}_{2}^{+}\right) 391.4$ and $(\mathrm{OI}) 557.7 \mathrm{~nm}$ emissions, i.e. the ratio $I_{6300} / I_{5577}>1$. According to Rees and Luckey (1974), such kind of auroral luminosity can be produced by precipitating electrons with energies from a few tens to a few hundred electronvolts, as it was confirmed as well by numerous spacecraft observations (e.g. Heikkila and Winningham, 1971; Heikkila et al., 1972; Frank and Ackerson, 1971). In the daytime sector, there is a distinct separation of precipitations into soft and hard zones, which was visualised by airborne and ground-based optical measurements (e.g. Heikkila et al., 1972; Vorobjev and Turjansky, 1983). Diffuse auroral luminosity with a ratio of $I_{6300} / I_{5577}<1$ exists equatorward of the dayside red band jointed to its equatorward edge. This luminosity, caused by an electron precipitation of a few kiloelectronvolts, is more distinct in the morning and pre-noon hours, but is also present in the afternoon, though much less intense there.

Figure 15 shows a scheme that summarises the principal peculiarities of the daytime luminosity structure in MLTMLat (magnetic latitude) coordinates. Noon (12:00 MLT) is in the upper part. This scheme was reported for the first time by V. G. Vorobjev on an international symposium in Suzdal (1986) and then published in the proceedings as a joint Norwegian-Russian report (Sandholt et al., 1988). Later, this scheme was shown in the paper by Leontyev et al. (1992): it corresponds to a mean level of magnetic activity and indicates the auroral luminosity regions with various spectral characteristics. The dominant $(\mathrm{OI})$ emissions in these regions are labelled as 557.7 or 630.0 . The dotted region in the noontime sector indicates soft electron precipitations with $I_{6300} / I_{5577}>1$. The direction of the discrete auroral form's motion is denoted by arrows. Beside of the motion of discrete forms toward the pole, there is an azimuthal motion from the noon sector toward dusk hours. 
In the pre-noon sector, a region with $I_{6300} / I_{5577}<1$ adjoins the region of soft electron precipitation from the equatorward side. This is a region of diffuse aurora, in which homogeneous arcs are moving towards the Equator. In the evening, auroral luminosity is mainly concentrated within the auroral oval and $I_{6300} \approx I_{5577}$. Near 15:00 MLT one can observe changes of the auroral type from rayed to homogeneous forms and of their meridional movement from poleward to equatorward.

A gap within the discrete auroral forms near noon is missing in Fig. 15. Such a gap was detected by spacecraft observations and it is usually treated as a permanent peculiarity of the daytime sector. However, ground-based all-sky cameras rarely observe a daytime auroral gap near noon.

Vorobjev et al. (1988a) have recognised three subtypes of poleward moving auroral forms. Different behaviour of the red-to-green $\left(I_{6300} / I_{5577}\right)$ intensity ratio during the lifetime of these forms was the basis for this selection. The temporal behaviour of (OI) 630.0 (heavy lines) and $557.7 \mathrm{~nm}$ (thin lines) emissions for these subtypes are shown in Fig. 16a-c from the meridian scanning photometer (MSP) data analysis. Figure 16a demonstrates the intensity variations which are attributed to rayed arcs observed mainly near (often just before) magnetic noon. The brightness of arcs quickly increases for about $3 \mathrm{~min}$ and then a slower decrease in intensity is observed. The auroral green line intensity $I_{5577}$ decreases faster than $I_{6300}$, confirming the diminishing spectral hardness of the precipitating electrons. The $630.0 \mathrm{~nm}$ intensity, being prevalent during the lifetime of arcs, rarely exceeds $1 \mathrm{kR}$, whereas the $557.7 \mathrm{~nm}$ intensity of $0.4-0.6 \mathrm{kR}$ is typically observed. These faint arcs could not be registered by spacecrafts with low sensitivity devices, while the midday auroral gap can be identified herewith.

The temporal variations of intensity, as shown in Fig. 16b, are attributed to rayed arcs, which are generally observed after noon. These variations are similar to those just prior to the initial period (1-2 min) of the arc generation, as considered above, with $I_{5577}>I_{6300}$. According to Rees and Luckey (1974), the energy of precipitating electrons is larger than $1 \mathrm{keV}$ during this short time interval. The third type (Fig. 16c) is related to the brighter afternoon arcs, the intensity of which often increases drastically for a short time. The significant increase especially in $I_{5577}$ occurs during such bursts. According to TV observations, these bursts are associated with an abrupt brightening of a small rayed auroral form segment, which moves along the form in an east-west direction and falls therefore into the field of view of a MSP device. The bursts last, as MSP records show, from several tens of seconds up to 1-2 min and are recurring in 6-7 min intervals, while the recurrence period of auroral forms is about 4 min.

The mean variation of $K=I_{6300} / I_{5577}$ for dayside auroral forms is shown in Fig. 17. The secondary minimum in $\mathrm{K}$ is connected with the auroral bursts. The smooth increase of $\mathrm{K}$ during the lifetime of auroral forms confirms the soft-

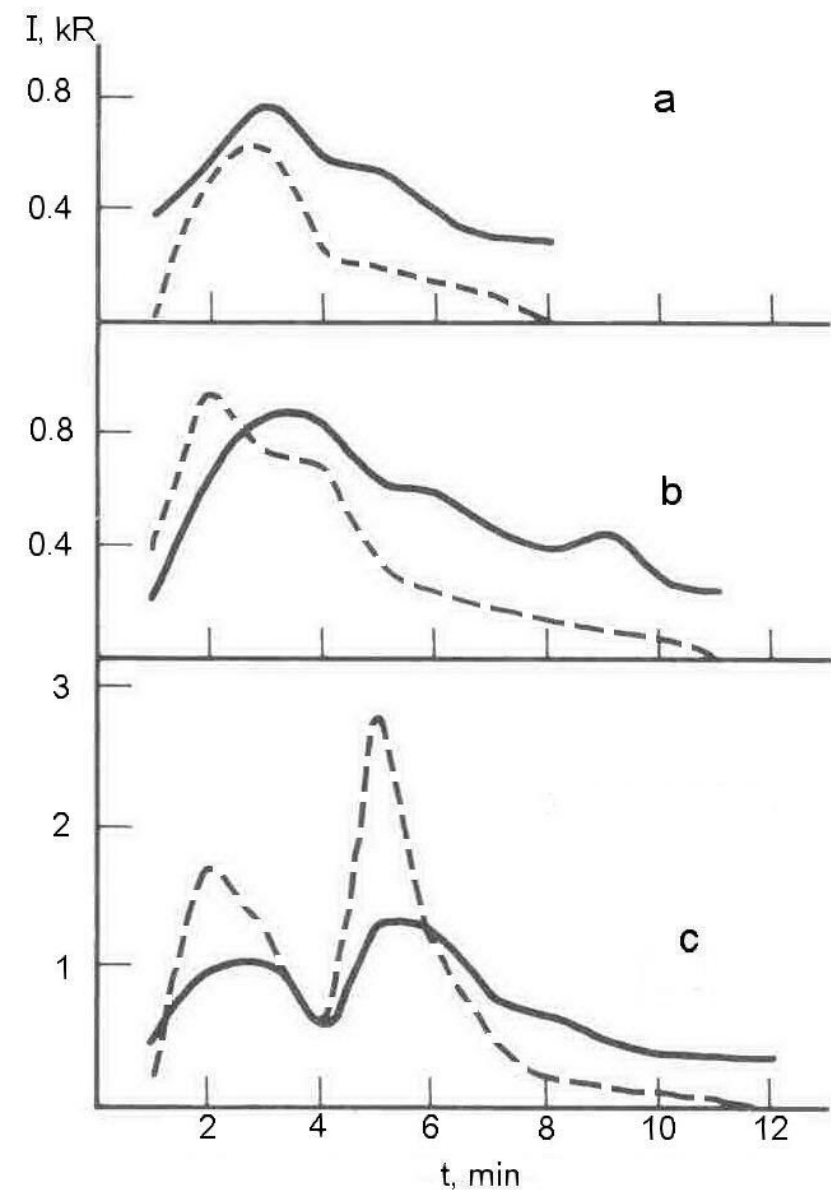

Figure 16. Temporal variations of (OI) 630.0 (heavy lines) and $557.7 \mathrm{~nm}$ (thin lines) emissions in different types of dayside auroral arcs (Vorobjev et al., 1988a). The three panels stand for various subtypes of rayed arc emissions: (a) near or just prior to noon, (b) just after noon, and (c) brighter afternoon emissions (see text for details).

ening of the precipitating electron energy spectrum and the subsequent uplift of the discrete forms during their poleward movement.

The ionisation profiles for different parts of the dayside ionosphere obtained with ionosonde observations at Heiss Island ( $\Phi^{\prime}=74.6^{\circ}$, Franz Josef Land) are shown in Fig. 18 (Vorobjev et al., 1990). $A_{1}$ and $A_{2}$ are the typical profiles observed in the region of diffuse auroral luminosity with a ratio $I_{6300} / I_{5577} \ll 1$ and a fairly high concentration in the E region, where a shielding $E_{\mathrm{sr}}$ layer is observed. The profile $\mathrm{B}_{1}$ corresponds to the equatorward part of the red auroral band, where the poleward moving auroral forms are observed, while the profile $\mathrm{B}_{2}$ corresponds to the poleward part of the red band generally without discrete auroras. The concentration of $B_{2}$ is much less than $B_{1}$ at the same altitudes. For the $\mathrm{B}_{1}$ and $\mathrm{B}_{2}$ profiles, the maximum of the electron concentration, which apparently corresponds to the maximum 


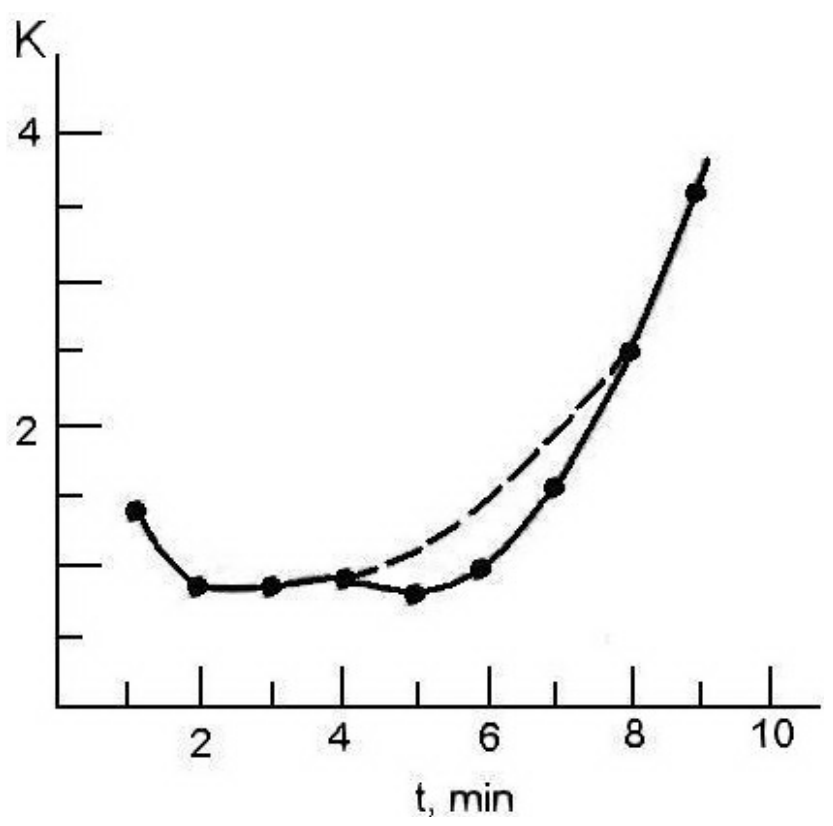

Figure 17. The mean variation of the red-to-green emission intensity ratio in dayside arcs (Vorobjev et al., 1988a).

of the red auroral luminosity, is located at an altitude of $\sim 250 \mathrm{~km}$. The profiles $\mathrm{C}_{1}$ and $\mathrm{C}_{2}$ are related to the polar cap area where the electron concentration is small with a maximum at $\sim 300-400 \mathrm{~km}$.

Sandholt et al. (1998) proposed several schemes, which differ from the one in Fig. 15, summarising principal peculiarities of the daytime luminosity structure and of the motion of discrete forms for various IMF orientations. These schemes, which are shown in Fig. 19, were constructed on the basis of MSP observations of 630.0 and $557.7 \mathrm{~nm}$ emissions and ASCs of $630.0 \mathrm{~nm}$ wavelength at the Ny Ålesund station, Spitzbergen $\left(\Phi^{\prime}=75.5^{\circ}\right)$. The sorting of the data was performed according to spectral and morphological auroral characteristics for various MLT sectors and IMF orientations. The various auroral forms are labelled with the numbers 1-7. The direction to the sun is downward in the figure.

Figure 19 shows the schemes for $B_{z}^{\mathbb{M F}}>0$ (a) and $B_{z}^{\mathbb{M F}}<0$ (b) under positive $B_{v}^{\mathrm{IMF}}$ conditions. The two other possibilities for the same $B_{z}^{\mathrm{IMF}}$ orientation, but under negative $B_{y}^{\mathrm{MF}}$ conditions is not shown. They differ from the schemes shown in Fig. 19 by the motion of discrete forms that are directed toward evening hours (eastward) instead of morning hours (westward).

The two main cusp auroral forms in the midday sector are Type 1 and Type 2 auroras which appear as rayed bands. Type 1 occurs at geomagnetic latitudes of $70-75^{\circ}$ and Type 2 at $75-79^{\circ}$. Their spectra is dominated by an emission of $630.0 \mathrm{~nm}$ with an intensity of $2-5 \mathrm{kR}$. Type 1 is often associated with poleward moving auroral forms (PMAFs). The azimuthal motion of the PMAFs is controlled by the $B_{y}^{\mathrm{IMF}}$ com-

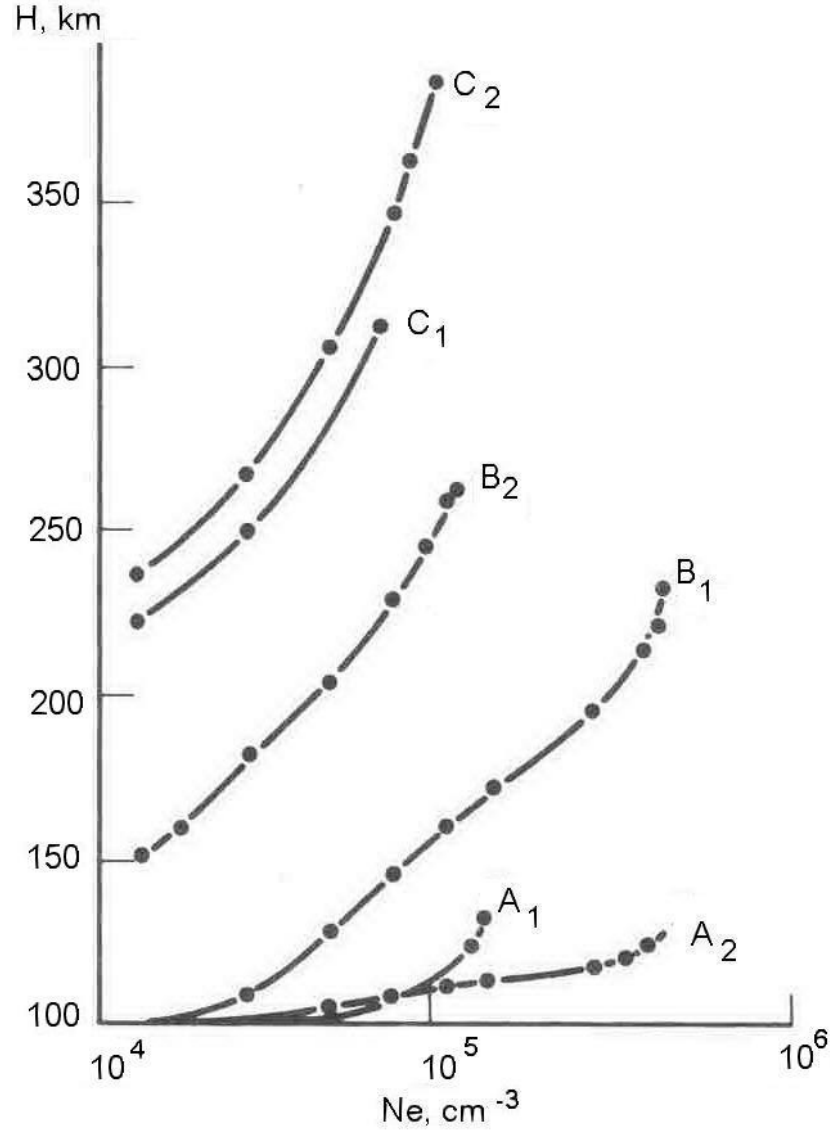

Figure 18. Ionisation profiles in different parts of the dayside ionosphere (Vorobjev et al., 1990).

ponent. Type 2 forms usually move equatorward and show also a $B_{y}^{\mathrm{IMF}}$ related east-west motion as indicated with the arrows.

The zone of diffuse auroras with $557.7 \mathrm{~nm}$ emissions (Type 3) comprise all MLT hours at lower latitudes. Types 2, 4 , and 5 are observed during positive $B_{z}^{\mathrm{MF}}$ periods, where short arcs of Type 5 extend toward evening hours, while Type 4 arcs extend toward morning hours. Type 1, Type 6 , and Type 7 are situated at lower latitudes.

Sandholt et al. (1998) assumed that the appearance of daytime Type 1 and Type 2 structures is caused by reconnection processes between the interplanetary and the magnetospheric magnetic field, i.e. within an open magnetosphere model. Structures of Type 4 and Type 5 or Type 6 and Type 7 are allocated within a closed magnetosphere.

The scheme of the distribution of dayside auroral forms and their dynamics was further elaborated in the study of Sandholt et al. (2002a). This new scheme is often cited in the scientific literature and represents a somehow modified version of the Sandholt et al. (1998) scheme.

Later Sandholt and Farrugia (2007) extended the categorisation of PMAFs. They classified them into four sub- 

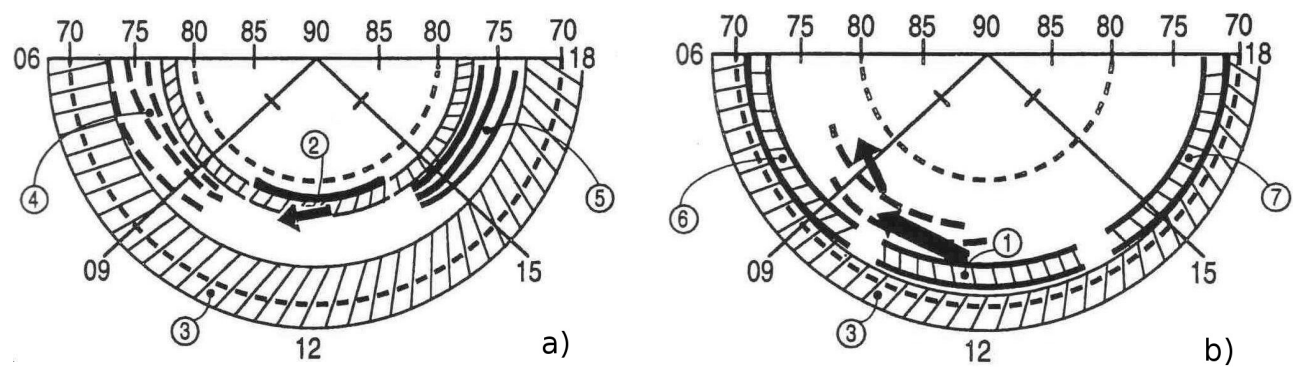

Figure 19. Schematic distribution of dayside auroras for different regimes of $B_{z}^{\mathrm{IMF}}$ and $B_{y}^{\mathrm{IMF}}$ components: (a) $B_{z}>0$ and $B_{y}>0,(\mathbf{b}) B_{z}<0$ and $B_{y}>0$. Seven different types of aurorae are marked by labels. Auroral motions Type 1 and Type 2 are indicated by arrows. The direction to the sun is downward in the figure (Sandholt et al., 1998).

categories according to the local time of occurrence and to the $B_{y}^{\mathrm{IMF}}$ component as follows: pre-noon $/ B_{y}>0$, prenoon $/ B_{y}<0$, post-noon $/ B_{y}>0$, and post-noon $/ B_{y}<0$. All subcategories occurred under a steady southward $\left(B_{z}<0\right)$ directed IMF, but with a dominant east-west $\left(B_{y}\right)$ component. Auroral observations were made at $630.0 \mathrm{~nm}$ by meridian scanning photometers and an all-sky camera in Ny Ålesund $\left(76^{\circ}\right.$ MLat). Ionospheric plasma convection was inferred from SuperDARN (Super Dual Auroral Radar Network) radars. Observations of ion drift and particle precipitations (electrons and ions) were obtained from DMSP F13 and field-aligned currents are based on measurements of the satellites FAST (Fast Auroral Snapshot) and DMSP F13. They found that the pre-noon and post-noon auroral forms are separated around noon by an attenuated $630.0 \mathrm{~nm}$ emission. The activation of PMAFs starts with an increase of the emission intensity at noon. Later stages of the PMAF evolution were examined in relation to plasma convection cells, flow vorticity and particle precipitation boundaries. The PMAFs are accompanied by the appearance of flow channels in the ionosphere, which are considerably strengthen by polarisation effects at the auroral boundaries. The existence of an $B_{y}^{\mathrm{IMF}}$ component leads to a considerable dawn-dusk asymmetry. This concerns the position of the dawn and dusk convection cells, the particle precipitations, and the configuration of the FACs. Owing to all this, there appears a dawndusk asymmetry of the PMAFs. The ion drift maximises at the poleward boundary of the auroral arcs. This increase of the flow speed constitutes a polarisation effect. The low conductivity on the poleward side of the arcs leads to an enhanced southward directed electric field, which drives the Hall current.

In general, the schema shown in Fig. 15 (Leontyev et al., 1992) essentially differs from that in Fig. 19a and b (Sandholt et al., 1998). Such a difference is self-evident because Fig. 15 illustrates the positioning of auroral forms during an average disturbance level, while Fig. 19 was constructed for periods of positive $B_{z}^{\mathrm{MF}}$ and negative $B_{z}^{\mathrm{IMF}}$. The main differences are those absent in Fig. 15, the Type 2 auroral forms observed during positive $B_{z}^{\mathrm{IMF}}$, and any influence of the $B_{y}^{\mathrm{IMF}}$ component on the east-west direction of poleward moving auroral forms. On closed field lines, an eastward movement of dayside rayed arcs corresponds to a tailward displacement of the arc's origins in the magnetospheric equatorial plane.

\section{Auroral dynamics during substorms}

\subsection{Auroral substorms}

Birkeland $(1908,1913)$ concluded from the analysis of the magnetograms, which he had obtained during Arctic expeditions organised by him, that the variation of the magnetic field at high latitudes consists of short and intense spikes, which succeed consecutively one after another. The spikes attain amplitudes from several hundreds to $10^{3} \mathrm{nT}$; their durations range from tens of minutes to several hours. Birkeland called them "polar elementary storms" of positive (the $\mathrm{H}$ component of the magnetic field increases in comparison with the quiet-time level) or negative kind (the $\mathrm{H}$ component decreases). The most intense polar elementary storms are observed at latitudes of the auroral zone.

Chapman (1935) carried out basic research on magnetic field structures during disturbances by generalising a large amount of observations. He assumed the high-latitude disturbance field, $D$, to consist of long-lasting regular components $S_{\mathrm{D}}$ (daily variation of geomagnetic disturbance, controlled by LT and UT) and $D_{\mathrm{m}}$ (aperiodic variations, controlled by UT and not dependent on longitude), but also of an irregular $D_{\mathrm{i}}$ part. The regular variations were assumed to represent a long-term feature, which are not connected to the non-regular oscillations of the geomagnetic field. The regular components $D_{\mathrm{m}}$ and $S_{\mathrm{D}}$ carry the main part of $D$, while $D_{\mathrm{i}}$ smoothes out during averaging and its intensity is much weaker than the regular contributions. For a long time after the publication of Chapman (1935), the majority of authors paid attention to the study of the $S_{\mathrm{D}}$ and $D_{\mathrm{m}}$ variations. The equivalent current system, constructed by Sydney Chapman (1888-1970), became the leading paradigm in the study of magnetospheric current systems.

Nikolsky (1956) and later Burdo (1960) analysed magnetic variations from high-latitude observatories with regard 
to the existence of regular $S_{\mathrm{D}}$ and $D_{\mathrm{m}}$ variations. Burdo (1960) determined the diurnal variation of the geomagnetic field elements for quiet hours during global magnetic storms for both the international quiet and disturbed days based on observations in Dixon Island $\left(\Phi^{\prime}=67.9^{\circ}\right)$ and Tikhaya Bay $\left(\Phi^{\prime}=74.4^{\circ}\right)$. The magnetic field turned out to be at a quiet field level during the hours of global storms, when they were free of irregular oscillations. No noticeable long-term variations could be discovered that were not related to irregular oscillations. The division of the $D$ field into $D_{\mathrm{m}}$ and $S_{\mathrm{D}}$ variations appeared to be a formal mathematical approach.

The regular variations of the geomagnetic field $S_{\mathrm{D}}$ and $D_{\mathrm{m}}$ at high latitudes are, therefore, almost completely created by the "polar elementary storms". The evolution of ideas about the structure of disturbance variations of the high-latitude geomagnetic field proceeded thus in the 20th century from polar elementary storms in the beginning of the century over large-scale regular $S_{\mathrm{D}}$ and $D_{\mathrm{m}}$ variations in the 1930 s to the reassumed conception of a sequence of short magnetic field spikes in the mid-20th century.

Such a conception about the structure of magnetic disturbances at high latitudes was adopted by the scientific community as a result of the IGY period. Thus, according to Chapman (1962), the magnetic disturbances consist of sporadic, rapidly emerging and decaying bursts (polar substorms) both during magnetic storms and during rather quiet periods.

The ideas about the succession of discrete auroral forms run approximately through an analogous evolution like those about magnetic disturbances. The polar elementary storms identified and defined by Kristian Birkeland were actually the magnetic disturbances, which come along with auroral activations. The study of disturbances developed in the course of the beginning 20th century primarily via observations of visual auroras and magnetic field fluctuations that accompanied changes in auroral luminosity. Birkeland (1913) discussed the possible nature of a current system that could be accounted for by the observed magnetic disturbances: the currents are located close to Earth or form a three-dimensional (3-D) current system with two vertical currents in opposite direction connected by a horizontal section. Birkeland pointed out that a current system with vertical currents can explain some of the properties of the polar magnetic disturbances. Bostrøm (1964) proposed 3-D current systems in two variants. Type I is a modified Birkeland current system, where the vertical currents flow along the curved geomagnetic field lines. Polarisation effects occurring in regions of enhanced conductivity lead to a southwarddirected electric field and to a strongly intensified westward Hall current. In Type II a Pedersen current flows southward across the electrojet and is closed by sheet currents at the southern and northern edges of the electrojet. The electrojet between the two sheet currents appears as a Hall current.

Akasofu (2004) assumed that the observed main features of auroral substorms could be explained quantitatively by the single fact that Bostrøm's current systems are activated at substorm onset. This comprises the following features:

1. A sudden brightening of the aurora due to an increase of the auroral electron flux or an intensification of the FACs.

2. A growth of the westward electrojet due to an enhanced southward electric field or Pedersen current.

3. Dipolarisation of the magnetic field due to a reduction of the cross-tail current.

However, the existence of an eastward electrojet in the ionosphere and its closure by FACs, which is the characteristics of the 3-D current system in the evening sector of the magnetosphere, is not taken into account in Bostrøm's current systems. Thus there emerged in the scientific literature on model descriptions of substorm currents also other concepts of current systems (see, e.g. Sergeev et al., 2014). The 3-D magnetospheric current system is controlled yet by several other sources and proves therefore to be quite complex. The relation of the current system to the various plasma domains in the magnetosphere is shown in Fig. 16 of Feldstein et al. (2006).

As the magnetic field measurements were easier to acquire than auroral observations on a global scale, they form the basis for most of the discussions, which lead to the present-day perception of the structure and evolution of planetary electromagnetic field disturbances.

Fuller (1935) and Heppner (1954) generalised the results of visual auroral observations at high latitudes of the western hemisphere and proposed the fixed auroral pattern model. The model includes the latitudes $60^{\circ}<\Phi^{\prime}<70^{\circ}$ and the 19:00-06:00 MLT interval, when visual observations are possible in Alaska. Auroras exist permanently, both during geomagnetically quiet and disturbance intervals, and their distribution is fixed with respect to the sun. A change of the forms for an observer at Earth's surface is caused by the diurnal planetary rotation about its axis. During evening hours one observes usually homogeneous arcs and a diffuse luminosity equatorward of them; pulsating forms and a diffuse luminosity dominate during morning hours. The auroras are most active around midnight, where they comprise a maximum latitude interval. The auroral forms and their positioning are closely related to geomagnetic field variations with an eastward current at dusk and a westward current during dawn hours. A change of the electrojet direction occurs at midnight, where the auroras are most intense, from eastward in the evening to westward in the morning hours.

Photographic aurora observations during the IGY allowed obtaining and analysing snapshot distributions of auroras and their dynamics over large areas. Akasofu (1963, 1964a) concluded from these analyses that the activation of auroras from the quiet state to the appearance of intense auroral forms and the subsequent decay proceed in cycles. Every cycle 


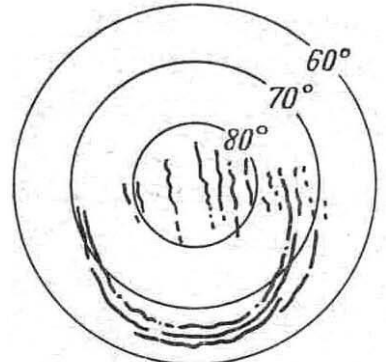

A. $T=0$

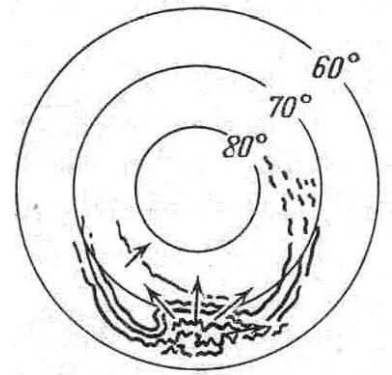

C. $T=3 \sim 10 \mathrm{MIN}$

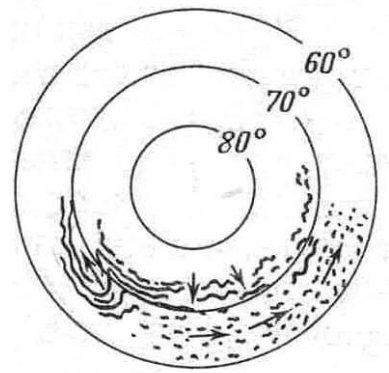

E. $T=30 \mathrm{MIN} \sim 1 \mathrm{HR}$

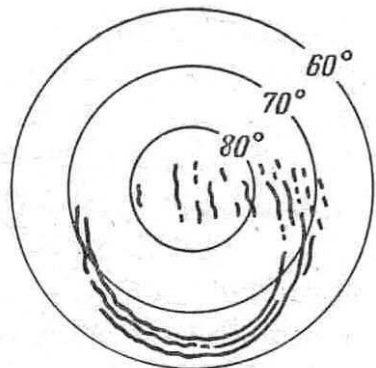

B. $T=0 \sim 3 \mathrm{MIN}$
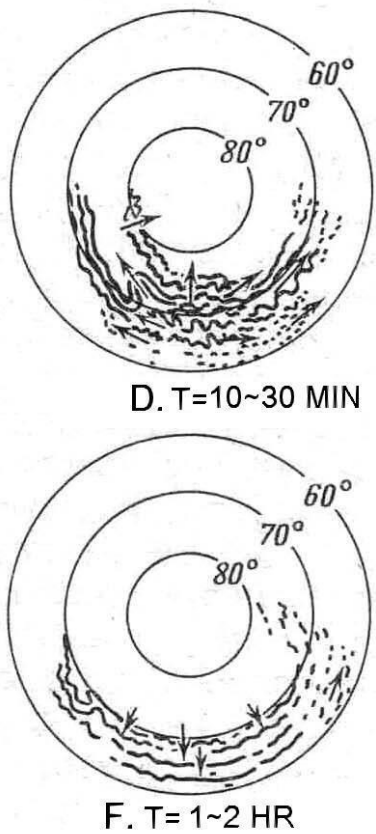

F. $T=1 \sim 2 H R$
Figure 20. Schematic diagram of substorm development for nighttime aurorae (Akasofu, 1964a).

constitutes an individual auroral substorm. The duration of substorms lasts between 1 and $2 \mathrm{~h}$; during nighttime substorms can recur several times. Figure 20 shows the changes of auroral luminosity during substorm development according to the pioneer work of Akasofu (1964a). The change of auroral forms for a ground-based observer occurs not only due to Earth's rotation under a quasi-stationary auroral pattern as it was proposed in the model of fixed auroral patterns, but to a great deal due to the expansion and decay of the disturbance. The development of auroral substorms occurs according to Akasofu (1964a) from a quiet state (moment $T=0)$ over the expansion phase $(0 \leq T \leq 30 \mathrm{~min})$ and the recovery phase $(30 \leq T \leq 120 \mathrm{~min})$ back to the quiet state.

The dynamics of discrete auroral forms during auroral substorms as it is shown in Fig. 20 (Akasofu, 1964a) comprises only the nighttime sector. This confinement is due to the use of observations from Alaska and Canada only. The study of auroras in the daytime sector requires the inclusion of observations from high latitudes of the eastern hemisphere, where visual auroral observations during daytime hours are possible close to the winter solstice. A scheme of substorms with two phases, which comprises all local time hours, was proposed by Feldstein and Starkov (1967b) and then also by Akasofu (1968).

This scheme of the auroral substorm was later considerably modified in the publications of Feldstein and Starkov (1970a) and Starkov and Feldstein (1971b) as shown in Fig. 21. A third phase ("creation phase") prior to the initial moment $T=0$ was introduced additionally to the expansion and recovery phases. During these years a third phase of substorm development in the magnetic field variations was proposed by McPherron (1970) under the notion "growth phase". The scheme of auroral substorms as shown in Fig. 21 proves to be the most complete generalisation about the conception of a single substorm development in the auroras and its description by Feldstein et al. (2010).

Gjerloev et al. (2007) determined how often auroral manifestations of polar substorms occur according to the scheme shown in Fig. 20 (Akasofu, 1964a), but in a somewhat simplified manner. They used imaging data of an Earth camera in the far ultraviolet (FUV) range of $124.0-149.0 \mathrm{~nm}$ that were supplemented by the $557.7 \mathrm{~nm}$ visible images from the visible imaging system (VIS) on board the Polar spacecraft (Frank et al., 1995). The selection of the events was based both on their optical characteristics and the AL index pattern around the time of events. Detailed descriptions of the criteria used for the selection of events during the winter seasons of the years 1997-2011 is given by Gjerloev et al. (2007) and Gjerloev et al. (2008). Disturbances were classified as substorms, if they were accompanied by a poleward bulge expansion from the auroral oval near midnight and by the development of an auroral surge in the evening sector to the west of the bulge. Gjerloev et al. (2007) found that the Akasofu type substorm is the predominant type of auroral events for isolated substorms. In 116 cases of all the selected events, the auroral disturbances developed according to the classical scheme of Akasofu (1964a), and only 20 further cases did not display the classical characteristics.

Utilising global auroral images obtained by the VIS, Gjerloev et al. (2008) developed a quantitative method to describe the spatio-temporal behaviour of auroras during the expansive and recovery phases of substorms. Resulting from the analysis of 116 substorms, they compiled latitudinal variations of structural auroral forms for each of 24:00 MLT hours and for 11 time steps of the substorm, covering 20 min prior to the onset until well into the recovery phase. They revealed key features of the individual auroral patterns, which form the background for the perception of a statistical substorm. Based on their statistical model of the auroral substorm, a bifurcation of the auroral oval into two components occurs just after the onset time. The equatorial component (the oval aurora) remains practically in the latitude range of the preonset oval, while the bulge aurora emerges out of the oval, expanding poleward, and both east and west in MLT. It was 

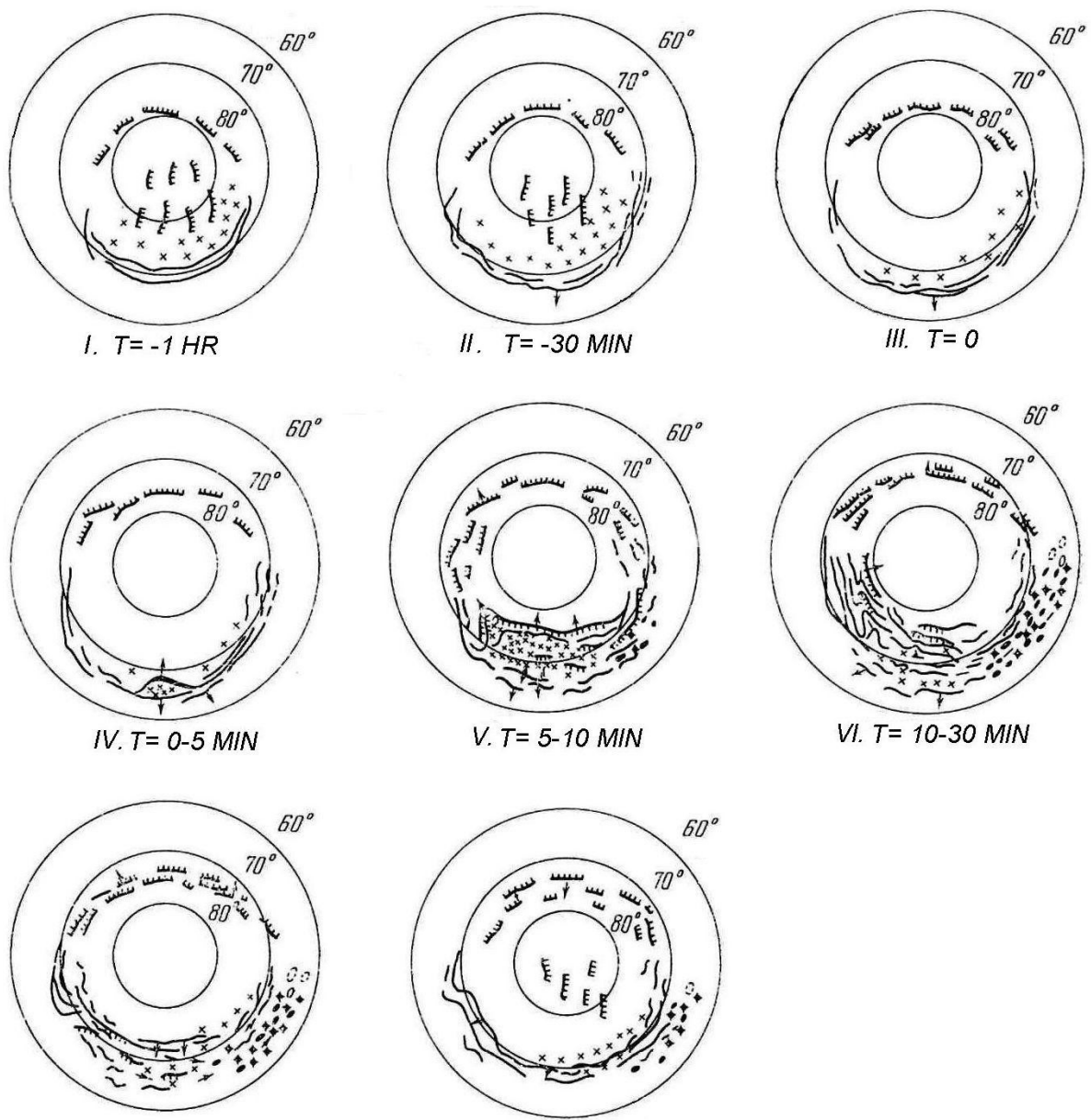

VII. $T=30 \mathrm{MIN}-1 \mathrm{HR}$

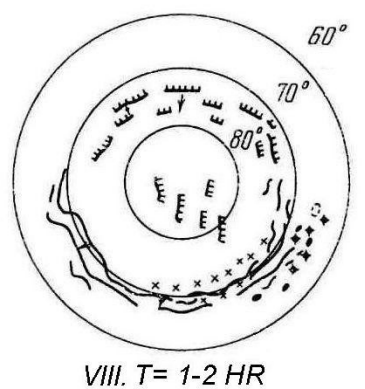

Figure 21. Planetary scheme of auroral substorm development (Feldstein and Starkov, 1970b; Starkov and Feldstein, 1971a).

found that the bulge aurora starts with a localised onset. A new region of emissions is created outside the pre-onset oval, whereas the oval aurora brightens simultaneously within the pre-onset oval over some hours in local time.

Auroral substorms occur both during relatively quiet conditions (as single substorms) and during magnetic storms, where an overlay of several substorms takes place in the main phase (the new substorm already starts while the preceding substorm is not yet terminated). This more complex scenario is referred to as a multi-cycle substorm. Spatiotemporal models of single substorms were described above and are presented in Figs. 20 and 21 (see also Fig. 28 later on). Several studies explored possible differences in the course of single auroral substorms and multi-cycle substorms. Tsurutani et al. (2003) investigated the evolution of the auroral luminosity using images of the Polar spacecraft during the main phase of magnetic storms that have been induced by magnetic clouds in the solar wind passing Earth's magnetosphere. They claim that the bulge is missing in the substorm expansion phase of multi-cycle substorms. The luminosity region, which comprises mainly patches, extends in north-south direction over a large longitudinal range. The intensity of the diffuse luminosity increases after the substorm onset, which affects the possible partition of structured forms at the satellite images.

Henderson et al. (2006a, b) performed detailed investigations of the dynamics of auroral bursts, based on images and keograms of auroras on board the Polar and IMAGE (Imager for Magnetopause-to-Aurora Global Exploration) satellites. They analysed the electron and ion energy flux variations at a series of geostationary satellites like GEOS (Goddard Earth Observing System) and LANL (Los Alamos National Laboratory) for the two magnetic storm intervals of 18 April 2002, and 10-11 August 2000. During these storm intervals they identified sawtooth events, which represent disturbances of the auroral luminosity and precipitating particles with a periodicity of 2-4h. These disturbances were termed sawtooth events because the temporal variations of the proton flux resemble in their shape the teeth of a saw blade.

Sawtooth disturbances are greatly similar to substorms, but the auroral activity rapidly engages a wider than usual azimuthal range and the injections can also be dispersionless 
over a much wider than usual range of local times (Henderson et al., 2006a). The auroral distribution in the nighttime sector has a double-oval configuration, which is observed during storm intervals and sometimes also during the recovery phase of individual substorms. The auroral disturbances begin with a fairly localised onset in the equatorward branch of the double oval in the evening sector and expand eastward, westward, and poleward. This is the expansion phase of the disturbance, prior to which occurs an equatorward motion of the poleward branch, i.e. a thinning of the double oval. This displacement can be considered as the creation (growth) phase. The creation phase is accompanied by a stretching of the nighttime magnetic field lines in antisolar direction and hence a thinning of the plasma sheet in the magnetospheric tail. The creation phase of each sawtooth comes along with a plasma injection at geostationary orbit and the formation of streamers between the equatorward and poleward branches of the double oval. There is no obvious appearance of a bulge during the creation phase of sawtooth disturbances, which are typical for individual auroral substorms.

Kornilov and Kornilova (2009) analysed ground-based television and satellite auroral images of the main phases of 10 storms. It turned out that the variation of auroral luminosity can take place in different scenarios: from absent bulges to scenarios with subsequent poleward shifts of an auroral arc, accompanied by a rapid broadening of a bright diffuse luminosity over all azimuths. Structured forms that belong to different substorm phases appear in the television camera field of view.

Hoffman et al. (2010) compared the optical signatures of substorms that occurred during the development of the main phase of magnetic storms (disturbed or multi-cycle substorms) with the optical signatures of isolated substorms as defined by Akasofu (1964a). For the selection of substorm intervals they used only optical observations of the Polar spacecraft in the far ultraviolet $(124-149 \mathrm{~nm})$, supplemented by $557.7 \mathrm{~nm}$ visible images. Auroral disturbances were identified as isolated substorms according to the following optical characteristics:

1. an emission region (bulge) arises in latitudes outside the auroral oval;

2. the presence of a bifurcation, separating the bulge aurora from the oval, and also of a surge on the head of the bulge;

3. an auroral oval offset to the east in MLT from the bulge aurora.

Within the years 1997-1999, a total of 116 isolated substorms were identified and included in the subsequent analysis. For the same interval, 54 events of multi-cycle substorms during 16 magnetic storms were identified. The intensity of the storms amounted to SymH (Symmetric H) index values (equivalent to the $D s t$ index) between -60 and $-120 \mathrm{nT}$.
The selection of events was based on following optical characteristics that could be identified in the Polar images:

1. an onset during the main phase of the selected storm

2. an onset distinguishable from any existing auroras

3. a brightening of the aurora and its spatial expansion

4. a new event distinguished from an ongoing event.

The characteristics of auroral activity during multi-cycle substorms are very dissimilar to those of isolated substorms. The differences between these two substorm types comprise:

1. multi-cycle substorms commence at lower latitudes and later in local time;

2. they have a shorter expansive phase and a large intensity;

3. they develop neither a surge/bulge nor a bifurcation;

4. the area of auroral luminosity broadens mainly equatorward, and only slightly poleward;

5. the broadening auroral region drifts eastward;

6. the variations in shapes and sizes are much larger for multi-cycle substorms in contrast to isolated substorms.

Hoffman et al. (2010) relate the specified differences between the two substorm types to the intensifications of the large-scale current system and the electric field of magnetospheric convection as well as to the restructuring of the plasma domains in the nightside magnetosphere during magnetic storms, to the more intense electron precipitations, and to the enhanced integrated conductivity of the ionosphere. The most significant differences of the two substorm types are the lack of surges/bulges and bifurcations in multi-cycle substorms. The absence of these substorm characteristics is interpreted by Hoffman et al. (2010) as a direct evidence for a changed 3-D current system during magnetic storm intervals that connects the magnetosphere and ionosphere. This comprises, in particular, the existence of a substorm current wedge that forms the ionospheric part of the FAC system. The Hall current system should therefore change, resulting in variations of the magnetic field at Earth's surface and in the ionosphere.

\subsection{Behaviour of the discrete auroral forms}

The appearance of magnetospheric disturbances is related to active processes at the sun. A significant amount of energy derived from the solar wind-magnetosphere interaction and stored within Earth's magnetosphere is deposited in the auroral ionosphere and in the magnetosphere during substorm processes (Rostoker et al., 1980). Usually substorms are preceded by a southward turning of the IMF, i.e. the 
$B_{z}^{\mathrm{IMF}}$ component becomes negative (Rostoker and Fälthammar, 1967; Fairfield, 1967; Foster et al., 1971; Iijima and Nagata, 1972). However, substorms are at times also observed during quiet periods with positive $B_{z}^{\mathrm{IMF}}$ (Akasofu et al., 1973). Lee et al. (2010) report on several substorms observed under positive $B_{z}^{\mathrm{IMF}}$ conditions, the intensity of which was at least as significant as that of typical substorms under moderate negative $B_{z}^{\mathrm{IMF}}$. These substorms occurred during the recovery phase of intense magnetic storms. Their occurrence is caused by energy dissipation, which was previously stored in the magnetospheric tail during the main phase of storms.

The occurrence of substorms during positive $B_{z}^{\mathrm{MF}}$ is an evidence of the fact that the beginning of substorm development phases is determined by processes, which proceed within the magnetospheric tail. Negative $B_{z}^{\mathrm{IMF}}$ is not always a necessary condition for triggering mechanisms. Further, it was shown that the processes of substorm generation and their intensity are controlled by the whole set of the interplanetary medium parameters, such as the IMF magnitude and orientation, the solar wind velocity and density, and so forth (Perreault and Akasofu, 1978; Kan and Lee, 1979). A nearly universal solar wind-magnetosphere coupling function was offered by Newell et al. (2007).

Inasmuch as the interaction processes between the solar wind and Earth's magnetosphere take place first of all at the dayside magnetopause, it is the dayside aurora that reacts first on any change of the conditions in the interplanetary medium. Vorobjev et al. $(1975,1976 b)$ were the first who carried out a detailed analysis of the dayside discrete auroral forms during periods of substorms. They also studied concurrently the dynamics of the dayside auroras according to observations of the Pyramida station $\left(\mathrm{PYR}, \Phi^{\prime}=74.8^{\circ}\right.$, MLT $=\mathrm{UT}+2.5)$ and the nightside auroras with observations from the eastern part of Russia and from Alaska during the IGY. Figure 22 illustrates typical auroral behaviour using the example of a substorm during the interval 08:00-13:00 UT on 18 December 1957 (Vorobjev et al., 1976b). The positions of the various auroral forms along the geomagnetic meridian were obtained every minute from all-sky cameras under the assumption that the auroral heights were at 150 and $110 \mathrm{~km}$ on the dayside and nightside, respectively. Lines connect in each case one and the same discrete auroral form. The nightside auroras were obtained from observations at the stations Wrangel Island (WRI, $\left.\Phi^{\prime}=66.6^{\circ} \mathrm{N}, \mathrm{MLT}=\mathrm{UT}+10.5\right)$ and College $\left(\mathrm{COL}, \Phi^{\prime}=64.9^{\circ} \mathrm{N}, \mathrm{MLT}=\mathrm{UT}+12.4\right)$. In order to eliminate the auroral movements associated with Earth's rotation under the oval, positions of daytime auroras (Fig. 22b) were determined from the oval centre for a mean magnetic activity level of $Q=4$. Figure 22a shows the daytime auroral intensity in arbitrary units integrated over the whole sky.

The onset time of substorm expansive phase $\left(T_{0}\right)$ near 11:00 UT manifested by the sharp intensification of nighttime auroras and the beginning of their poleward movement is denoted by an arrow and a vertical dashed line. A pro-

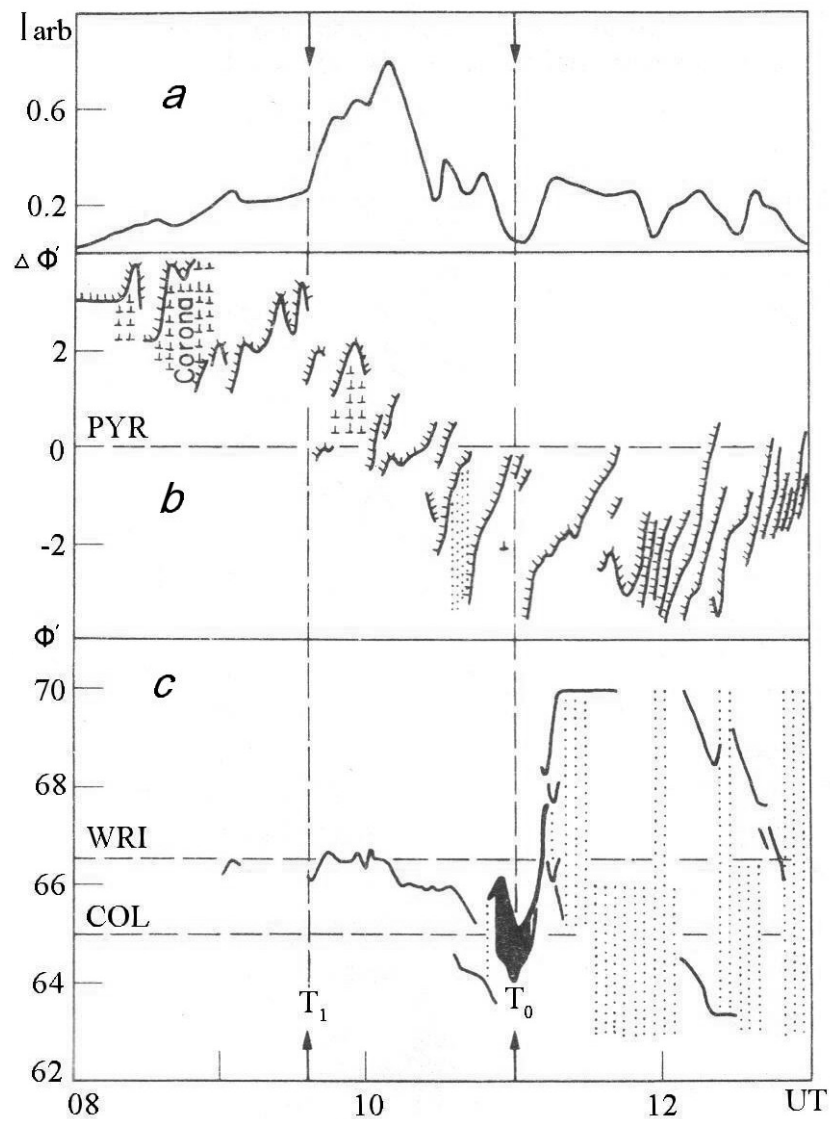

Figure 22. Dynamics of the daytime (station Pyramida, PYR) and nighttime (stations Wrangel Island, WRI, and College, COL) aurorae on 18 December 1957: (a) the daytime auroras' brightness in arbitrary units; (b) and (c) the auroras' development versus UT over daytime and nighttime stations, respectively. Horizontal dashed lines are the zenith of the observatories. The positions of homogeneous arcs are shown by solid lines; that of rayed ones by herringbone, and the diffuse luminosity by dots (Vorobjev et al., 1976b).

nounced equatorward displacement of dayside auroras was observed before $T_{0}$. The beginning of this equatorward shift $\left(T_{1}\right)$ at about 09:35 UT, i. e., about $85 \mathrm{~min}$ before $T_{0}$, is likewise indicated in the figure by an arrow and a dashed line. The dayside auroral arcs moved poleward, however, each subsequent arc appeared more equatorward than the previous one.

The interval $T_{1}-T_{0}$ is discussed by Vorobjev et al. (1976b) as a substorm creation (growth) phase. At the nightside just after $T_{1}$, the appearance of a homogeneous auroral arc was observed, which moved gradually equatorward up to $T_{0}$. The beginning of the creation phase was accompanied by a significant dayside auroral intensity increase (Fig. 22a). Another increase of dayside aurora brightness was observed just after $T_{0}$. Earlier the dayside aurora intensity increases before and just after the substorm onset time as discovered by Starkov 
et al. (1973). Near the $T_{0}$ moment, Fig. 22a shows a fading of the dayside aurora. This fading of the dayside aurora just prior to the substorm onset was studied in more detail by Vorobjev et al. (1992).

The equatorward displacement of the dayside auroral region is closely related to the southward turning of the $B_{z}^{\mathrm{IMF}}$ component (Vorobjev et al., 1976b, 1988b; Horwitz and Akasofu, 1977; Sandholt et al., 1983). The beginning of this dayside auroral displacement has a time lag of 10-15 min with respect to the $B_{z}^{\mathrm{IMF}}$ component turning (Vorobjev et al., 1976b). Considering this circumstance, the interval $T_{1}-T_{0}$ corresponds to the time where a negative $B_{z}^{\mathrm{IMF}}$ component exists prior to the onset of the substorm and, therefore, to the time of energy storage in the magnetospheric tail. The paper of Vorobjev et al. (1976b) considers 14 individual substorms. According to this modest statistics, the average duration $T_{1}-T_{0}$ amounts to $50 \mathrm{~min}$, during which the dayside aurora moves by $4^{\circ}$ on average toward the Equator.

The movement of the nightside discrete auroral forms has been described by many researchers. But only the auroral observations by means of ASCs during the IGY allowed Akasofu (1964a) to generalise the movement of the auroral structures. This composed scheme is reproduced in Fig. 20 and a large number of publications address the detailed description of the dynamics of discrete auroral forms during substorms (see, e.g. Akasofu, 1965). This scheme endured examination over times and was confirmed by records of the auroral dynamics both from ground-based observations and from the satellites ISIS (Anger et al., 1973), Dynamics Explorer (Frank et al., 1982), Viking (Cogger et al., 1988), and others with UV imagers on board.

Starkov et al. (1971) considered the movement of discrete forms with data of a large number of auroral substorms, originating in the evening-nighttime sector after long-lasting magnetically quiet intervals. They used observations of a meridional ASC chain during the IQSY. Figure 23 shows as an example the movements of auroral forms based on the analysis of ASC records in Cape Chelyuskin $\left(\Phi^{\prime}=71.2^{\circ}\right)$ for the substorm of 17 December 1965. Figure 23b presents the $\mathrm{H}$ component variations of the geomagnetic field obtained there and Fig. 23c shows the magnitude and direction of the movement of auroral forms. Velocities larger than $1.8 \mathrm{~km} \mathrm{~s}^{-1}$ are hallmarked by arrows.

The geomagnetic field at the observatories' locations was quiet or only slightly disturbed between 14:30 and 15:35 UT, although the discrete auroral forms moved quite rapidly southward, which is typical for the creation phase of an auroral substorm. Magnetic disturbances commenced at 15:35 UT, accompanied by a rapid shift of the auroral arc toward the pole, which is characteristic for the beginning of the substorm expansion phase. This was also accompanied by an increasing brightness and expansion of the auroral area. A ceasing poleward movement of the active auroral arc, its decreasing intensity and the diminishing auroral area indicated

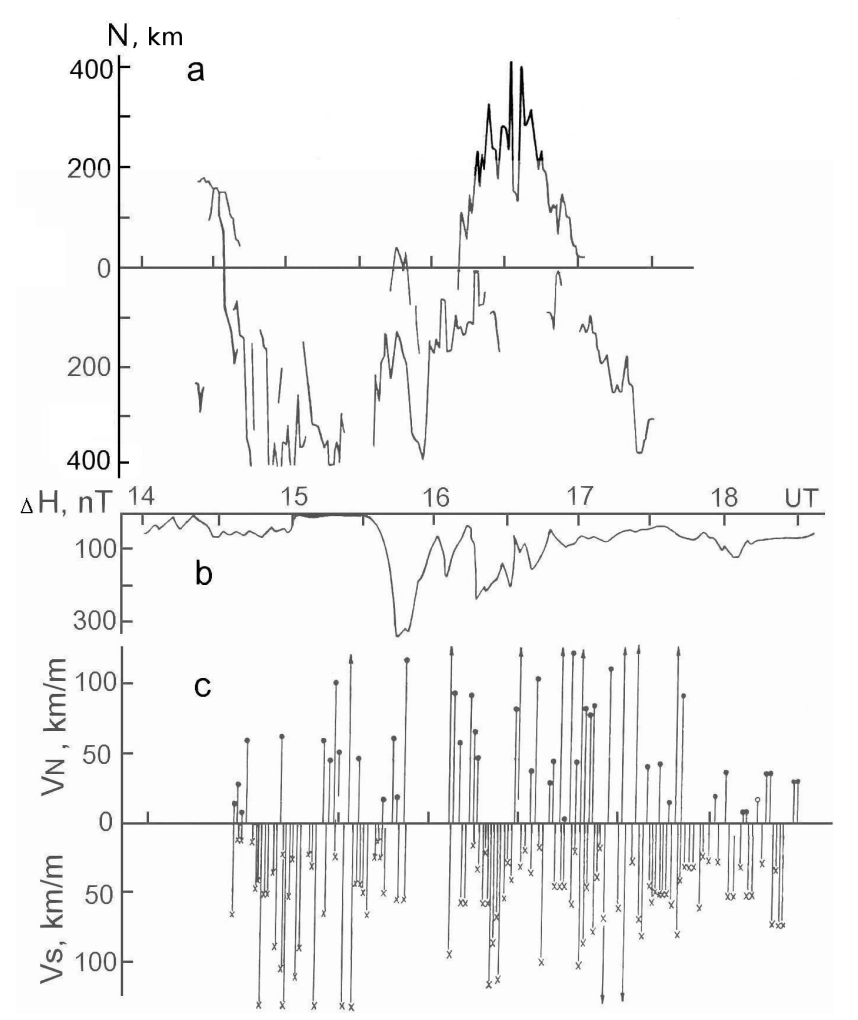

Figure 23. Development of an auroral substorm over Cape Chelyuskin on 17 December 1965: (a) dynamics of the auroral forms, (b) variation of the geomagnetic $\mathrm{H}$ component, and (c) magnitude and direction of the auroras' velocity, where the arrows show velocities beyond threshold (Starkov and Feldstein, 1971b).

the end of the active phase and the begin of the recovery phase (16:40 UT).

The distance of the auroral oval's equatorial boundary from the pole increases during the substorm creation (growth) phase at all local times (cf. Fig. 21) and the discrete auroral forms disappear within the polar cap. In the afternoon-evening sector, where usually one to three auroral arcs exist, an increasing equatorward movement of the discrete forms is clearly seen. Usually the whole oval moves equatorward, not only its equatorward part. During a typical substorm with a moderate intensification of the auroral luminosity, the nighttime sector of the oval moves equatorward by about $\sim 5^{\circ}$ down to $\Phi^{\prime} \sim 65^{\circ}$. Such a movement explains why the active phase starts during nighttime hours of the schemes in Figs. 20 and 21 just at this magnetic latitude.

Situations, where substorms occur without a distinct creation phase, are possible. This can occur if discrete auroral forms in the nighttime sector are still kept there from the previous recovery phase.

Snyder and Akasofu (1972) studied the movement of discrete auroral forms using observations of a meridional chain of ASC's in the western hemisphere $\left(76^{\circ}>\Phi>65^{\circ}\right)$. The discrete forms of the evening sector move regularly 
equatorward at $\sim 0.7^{\circ} \mathrm{h}^{-1}$ during quiet geomagnetic conditions due to their position along the oval. Prior to the start of the active substorm phase, the velocity of the arc's movement increases up to $\sim 4.0^{\circ} \mathrm{h}^{-1}$. Such an increase is characteristic for the creation phase. The velocity of the equatorward auroral motion may have the same magnitude as the velocity of the subsequent poleward expansion. The clearly observable systematic equatorward motion of the discrete auroral forms in the evening hours is brought about by two causes: the rotation of Earth under the auroral oval that is asymmetric with respect to the geomagnetic pole and the movement of discrete forms within the oval. The velocity of the arc's motion due to Earth's rotation is $\sim 1^{\circ}$ in latitude for $1 \mathrm{~h}$ and the relative motion within the oval amounts to $\sim 5^{\circ}$ (Feldstein, 1974; Feldstein et al., 2011). Up to $\sim 1 \mathrm{~h}$ before the start of the active substorm phase, an intensification of the magnetospheric dawn-dusk electric field takes place. This apparently supports the earthward approach of the central plasma sheet, which projects along the magnetic field lines to the nighttime sector of the auroral oval.

The creation phase of isolated substorms finishes usually with one single arc in the sector around midnight. The start of the expansion phase $\left(T_{0}\right)$ is determined as the moment of a sudden brightening of this arc in a wide longitudinal range (Figs. 20, 21). A splitting into flashing arcs (or the appearance of new arcs) takes place within a few minutes, which are violently moving poleward with a somehow more intense luminosity in the poleward part. An auroral bulge is developing with discrete forms that propagate rapidly poleward, westward (travelling surge) and eastward ( $\Omega$ band). The most intense luminosity is observed at the poleward border within the bulge. A somehow weaker arc remains at the place of the primary arc, which moves equatorward. The resulting bright area in the nighttime sector that is framed by discrete auroral forms extends both poleward and equatorward and comprises the maximum of the expansion phase $\sim 12^{\circ}$ in latitude.

The development of a new substorm can commence even under the presence of discrete forms that were kept there from the previous disturbance. During such situations in the expansion phase, the equatorward arc is the most active and most rapidly moving portion of the auroral oval (Akasofu et al., 2010). The poleward arcs do not play an active role for the substorm development and are absorbed by the poleward moving auroral bulge. Hence, although the start of the expansion phase is related to the equatorward arc, the most active band for the substorm development is located at the high-latitude poleward boundary of the bulge.

Based on event and statistical analyses of THEMIS all-sky imager data, Nishimura et al. (2010) showed that there exist qualitatively different sequences of events leading to the substorm onset. Such sequences are initiated by a poleward boundary intensification (PBI) and followed by a northsouth arc moving equatorward toward the onset latitude. PBIs have been related to enhanced flows that carry plasma across the nightside separatrix (De La Beaujardiere et al.,
1994) into the plasma sheet. Some PBIs develop into equatorward moving auroral arcs with roughly north-south orientation, also called "auroral streamers" (e.g. Sergeev et al., 1999). Because of this linkage, the results of Nishimura et al. (2010) indicate that substorm onsets are preceded by enhanced earthward plasma flows associated with an enhanced reconnection near the pre-existing open-closed field line boundary. The auroral observations indicate that the earthward transport of the new plasma lead to a near-Earth instability and an auroral breakup $\sim 5 \mathrm{~min}$ after PBI formation.

Auroral FUV images from satellites, which cover the whole high-latitude region, allowed determining the average MLat (deg) and MLT (h) positions, where the substorm's active phase usually commences. These coordinates of the auroral onset location was determined from the Viking satellite at $65.8^{\circ}$ MLat and 22.8 MLT (Henderson and Murphree, 1995), from the IMAGE satellite at $66.1^{\circ}$ MLat and 22.9 MLT (Frey et al., 2004), and from the Polar satellite at $66.3^{\circ}$ MLat and 22.9 MLT (Liou, 2010). The distributions of onset locations are near Gaussian with standard deviations of $2.2^{\circ}$ in latitude and $1.1 \mathrm{~h}$ in MLT, which quite satisfactorily agrees with the coordinates of the auroral substorm onsets as shown in Figs. 20 and 21.

The comprehension of auroral processes around the expansion phase onsets was improved with a series of further studies. Thus Elphinstone et al. (1995) analysed multiinstrument data sets from the ground and from satellites. Analysing 37 substorm events, where 26 of them were in a state prior to the explosive poleward auroral motion, they found the simultaneous occurrence of magnetic field Pi1 pulsations and azimuthally spaced auroral forms (AAF). The AAF elongations ranged from 132 to $583 \mathrm{~km}$, their average onset coordinates were at $63.8^{\circ} \pm 3.3^{\circ}$ MLat and $22.9 \pm 1.1$ MLT. AAF onsets occur during periods when the solar wind pressure is relatively high. The appearance of AAFs prior to the moment $T_{0}$ led to the conclusion that they are part of the creation phase activity. Based on observations from a comprehensive network of ground geomagnetic stations, Rae et al. (2009) identified the location of an epicentre for the generation of Pi1 magnetic pulsations, which appeared $\sim 2.5 \mathrm{~min}$ prior to the development of the auroral bulge. This epicentre is collocated with the region where spatially localised, latitudinally narrow small-scale $(\sim 70 \mathrm{~km})$ undulations develop on a faint isolated arc. This arc is located equatorward of the preexisting discrete auroral arcs. The poleward discrete arc is spatially distinct from the dynamic auroral activity, which originated in the epicentre of magnetic disturbances. A subsequent analysis of the frequencies of the magnetic oscillations, their spatial scales, and the growth rates of auroral forms resulted in the finding, that the onset phase of the substorm is most likely triggered close to the inner edge of the ion plasma sheet (Rae et al., 2010).

Sometimes substorm-like disturbances develop at nightside oval latitudes, which exhibit auroral activations without a clearly developed expansion phase. These events are 
characterised as pseudo-breakups (Koskinen et al., 1993; Nakamura et al., 1994). The dynamics of auroral pseudobreakup structures, their relation to the plasma processes in the magnetospheric tail and at the geostationary orbit were considered by Yahnin et al. (2001). They assumed that the main difference between substorms and pseudo-breakup activations consists in the different level of particle energisation within the magnetotail and in the auroral acceleration region.

Sergeev and Yahnin (1979a) described events where the auroral bulge was created by continuous deformations of auroral arcs, whose intensity steeply increased at substorm onset. Such forms of auroral activity result directly from the scheme of Akasofu (1964a). Analysing similar events, Sergeev and Yahnin $(1978,1979$ a) have concluded that in all cases the magnitude of magnetic disturbances in the auroral zone and the degree of the auroral bulge development were insignificant $\left(\sim-100 \mathrm{nT}\right.$ and $1-2^{\circ}$ of latitude, correspondingly). The authors argued that the bulge development during intense substorms and microsubstorms have different patterns or types. Using all-sky camera observations from a dense network of stations and riometer data to examine the bulge development, they found out that large expansions emerge generally from new bright arc formations poleward of the expanding bulge.

Figure 24 shows an example of an auroral expansion during the substorm of 13 February 1958 (Sergeev and Yahnin, 1979a). The position of regular auroral structures on the geomagnetic meridians of Point Barrow $\left(\Phi^{\prime}=69.9^{\circ}\right)$ and Fort Yukon $\left(\Phi^{\prime}=67.0^{\circ}\right)$ as it is seen in the field of view with a radius of $400 \mathrm{~km}$ are plotted here by solid lines. The height of auroral forms was assumed to be $110 \mathrm{~km}$. The vertical bars indicate extended bright regions, where it was impossible to distinguish more fine structures from the ascafilms. The development of the auroral bulge is realised by formation of new arcs in a poleward distance of $50-100 \mathrm{~km}$ from already existing auroral structures, as can be seen in Fig. 24. The study of a large number of such events showed the existence of discrete steps in the development of an expanding bulge, which are separated from each other by about 1-3 min.

A multiplicity of examples for the step-like development of the expanding bulge can be found in the literature. So, Lassen et al. (1977) have examined a big substorm during which an auroral bulge displacement of $9^{\circ}$ MLat was observed. The authors wrote that new auroral arcs were formed poleward of the pre-existent bright edge of the auroral bulge. Akasofu (1972d) pointed out that the formation of new arcs at the front of the expanding bulge is a typical phenomenon for the expansion in the pre-midnight sector. A similar description of the pre- and after-midnight events can be found in the papers of Akasofu et al. (1965), Oguti (1975) and others.

The moments of new arc formations can be traced also in other geophysical data. Such observational moments are, e.g. the increase of riometer absorption and changes in the regime of $\mathrm{Pi} 2$ pulsations (with respect to phase, polarisation,
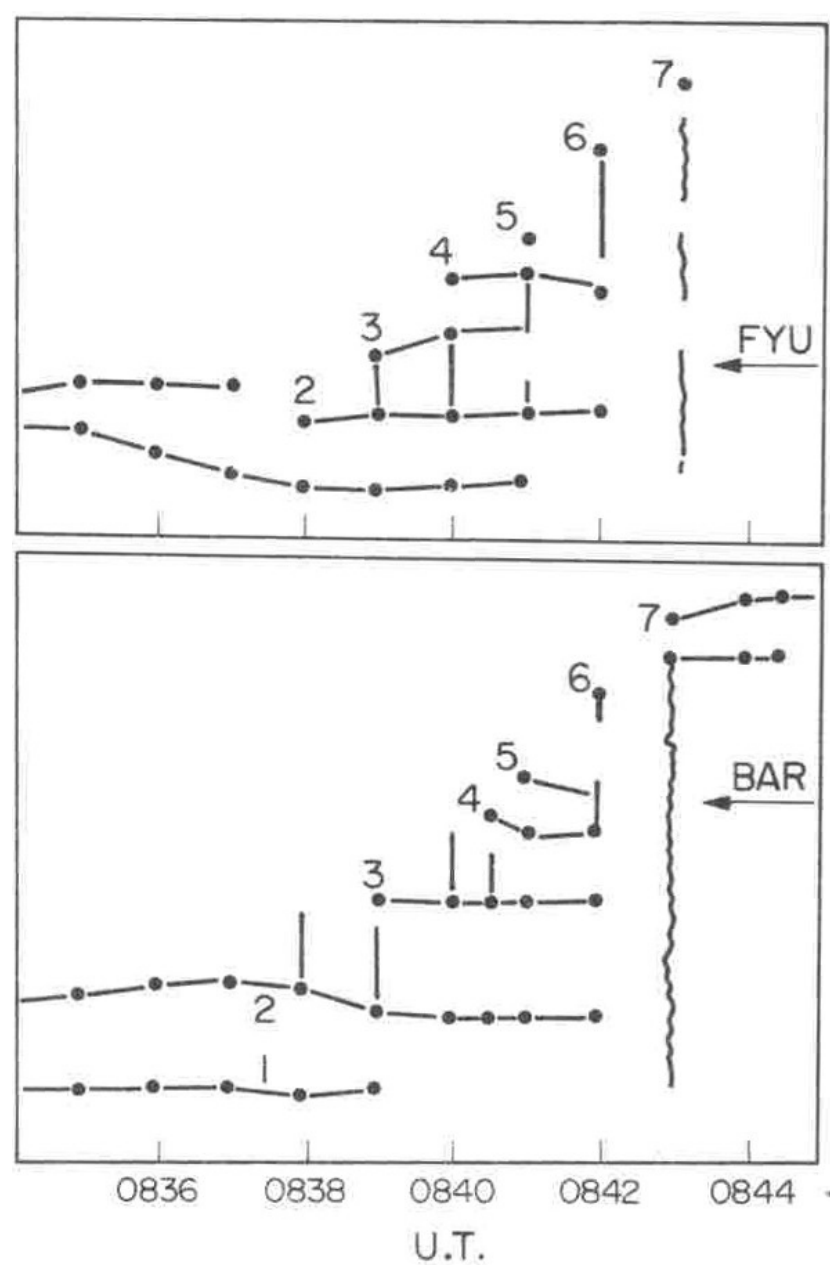

Figure 24. Distribution of discrete auroras at Fort Yukon and Point Barrow meridians during the substorm on 13 February 1958 (Sergeev and Yahnin, 1979c).

or amplitude) at high and midlatitudes (Sergeev and Yahnin, 1978, 1979a). During each new arc formation impulsive acceleration and precipitation of energetic electrons takes place and brief changes of plasma sheet geometry are sometimes observed at $18 \mathrm{Re}$ in the magnetotail (Sergeev and Yahnin, 1979a).

Simultaneous observations of the Double Star, Cluster, Geotail and five geostationary LANL satellites revealed according to Sauvaud et al. (2012) that the dynamics of changes in the plasma sheet and of the geomagnetic field in the magnetospheric tail repeats every $2-4 \mathrm{~h}$. The analysis of these results allowed determining the approximate location in the tail where the dipolarisations are initiated and the propagation direction of the disturbances. Each substorm complies with an energy-loading period, which is then followed by a dipolarisation at geocentric distances between 11 and $18 R_{\mathrm{E}}$. Plasma sheet thinning inside $12 R_{\mathrm{E}}$ occurs during the energy loading and is even enhanced during the onset of strong dissipations of magnetic energy. They precede 
the plasma particle injections at the geostationary orbit of $6.6 R_{\mathrm{E}}$. Sauvaud et al. (2012) showed that the onset of the magnetic energy conversion occurs at a tailward distance of $10-11 R_{\mathrm{E}}$ and propagates then both toward the Earth and toward the distant magnetospheric tail. According to Pudovkin et al. (1991), the creation phase is dominated by an enhancement of the dawn-dusk electric field, magnetic field stretching, and plasma sheet thinning. The initial expansion phase activity is characterised by magnetic field dipolarisations.

Substorms have their maximum latitudinal extent of the auroral expansion usually at the end of the expansion phase and during the beginning of the recovery phase. Processes of restoring the auroral distribution proceed during the recovery phase resulting in the initial pre-substorm state. The disintegration of structured forms on the nightside to diffuse auroras is a characteristic attribute of the auroral distribution according to Fig. 21 during the expansion and recovery phases of a substorm. The discrete forms vanish in the central part of the oval, while being preserved at its borders. Such regions of diffuse aurora are clearly seen at ASC images (Feldstein, 1972) and MSP (meridian scanning photometer) data (Akasofu et al., 2010) as dark areas during the expansion and recovery phases. This phenomenon is known as "double oval". It was studied in detail by Elphinstone et al. (1995). The discrete forms at the equatorward and poleward boundaries of the auroral oval can occur quite independently with respect to both appearance and intensity. They map along the magnetic field lines into different regions of the central plasma sheet of the magnetospheric tail. The double oval is observed only during expansion and recovery phases, but it is not characteristic for the creation phase, as it was summarised by Akasofu et al. (2010).

The motions of auroral forms and its velocity during various substorm phases were found out by Feldstein et al. (1971) for the evening-nighttime interval. Vorobjev et al. (1976b) determined separately the velocities of the arcs in different local time sectors for three substorm phases. They eliminated the shift of discrete auroral forms due to Earth's rotation under the auroral oval.

Feldstein et al. (1971) assembled histograms of meridional velocities for the various auroral forms. The quiet period preceding the moment $T_{0}$ (creation phase) is most often characterised by aurora velocities around $\sim 180 \mathrm{~m} \mathrm{~s}^{-1}$ with a strong dominance of equatorward motions. The number of fast motions of auroral forms increases with the start of the expansion phase both poleward and equatorward with prevailing velocities in the range of $300-600 \mathrm{~m} \mathrm{~s}^{-1}$. A strong increase is recorded in the number of poleward motions of $500-750 \mathrm{~m} \mathrm{~s}^{-1}$. Alongside with these motions, in particular within the high-latitude part of the oval, a few auroral forms move equatorward. Two maxima are formed within the histogram range $\pm 250-500 \mathrm{~m} \mathrm{~s}^{-1}$ due to motions with opposite directions. This opposite motion of arcs during the active phase of substorms is obviously caused by the simultaneous occurrence of two different processes within the plasma sheet of the magnetospheric tail: plasma motion toward Earth under the influence of the dawn-dusk electric field (equatorward motion of the arcs) and activation of new regions in the periphery of the plasma sheet (poleward motion of the arcs). The average velocity of the discrete forms diminishes to about $200 \mathrm{~m} \mathrm{~s}^{-1}$ during the recovery phase with a dominance of the equatorward motion.

The histogram of meridional velocities of discrete auroral forms according to Vorobjev et al. (1976b) is shown in Fig. 25. The mean velocities and the total number of events are indicated for each distribution. The creation phase is characterised by an equatorward shift of the auroral oval during daytime (09:00-15:00 MLT) and evening hours (15:00-21:00 MLT), but individual forms within the auroral region move poleward with an average velocity of $\sim 230 \mathrm{~m} \mathrm{~s}^{-1}$ northward during daytime hours and southward during the evening. Southward motion of about the same velocity dominates during nighttime and morning hours. The daytime forms drift poleward with an average velocity of $\sim 300-330 \mathrm{~m} \mathrm{~s}^{-1}$ during the expansion and recovery phases. Individual forms move both poleward and equatorward in the evening sector with average velocities of $\sim 160 \mathrm{~m} \mathrm{~s}^{-1}$ during the expansion phase, but during the recovery phase, in contrast to this, poleward at $\sim 200 \mathrm{~m} \mathrm{~s}^{-1}$. The average velocity of discrete forms increases sharply during the expansion phase in the midnight hours in poleward direction, while equatorward motions are maintained at a remarkable level (up to $370 \mathrm{~m} \mathrm{~s}^{-1}$ ). Velocities of $\sim 300 \mathrm{~m} \mathrm{~s}^{-1}$ are noticed in the morning hours during the expansion and recovery phases of substorms, which are both poleward and equatorward. Based on the velocity histograms of discrete auroral forms, Vorobjev et al. (1976b) proposed a scheme of ionospheric convection during the creation and recovery phases of substorms.

The use of the television technique became an important tool for auroral observations during recent years. It allows the recording of very faint, even subvisual emissions. Effective methods of filtering the television images were used for the data analysis, which even enable the study of fine structures and the auroral dynamics during substorms. In this way Kornilova et al. (2006) investigated the structure, dynamics, and correlation of auroral intensifications within the high-latitude and low-latitude boundaries of the auroral oval during substorm intervals. Kornilova et al. (2008) examined with 60 auroral substorms the relation between auroral activity in the different parts of the auroral oval near the onset of the substorm expansion phase. Kornilova and Kornilov (2012), finally, described the concurrent existence of equatorward drifting structures in the pole-side part of the nighttime auroral oval and poleward drifting of bright auroral forms in the equator-side part. The repetitive equatorward moving auroral forms between the two branches of the double-oval configuration were examined also by Sergeev et al. (1999), Sandholt et al. (2002b), Nishimura et al. (2010), and others. 
(a)
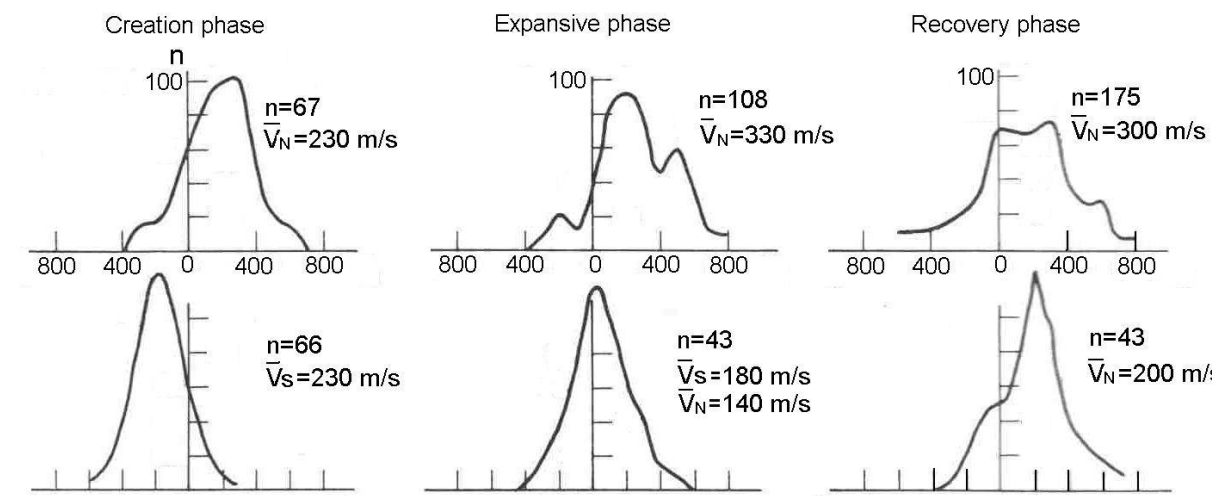

(b)

(c)

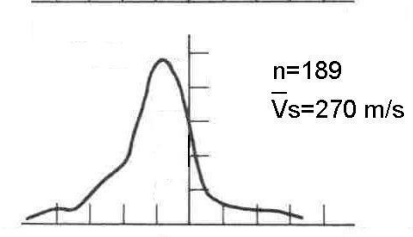

(d)
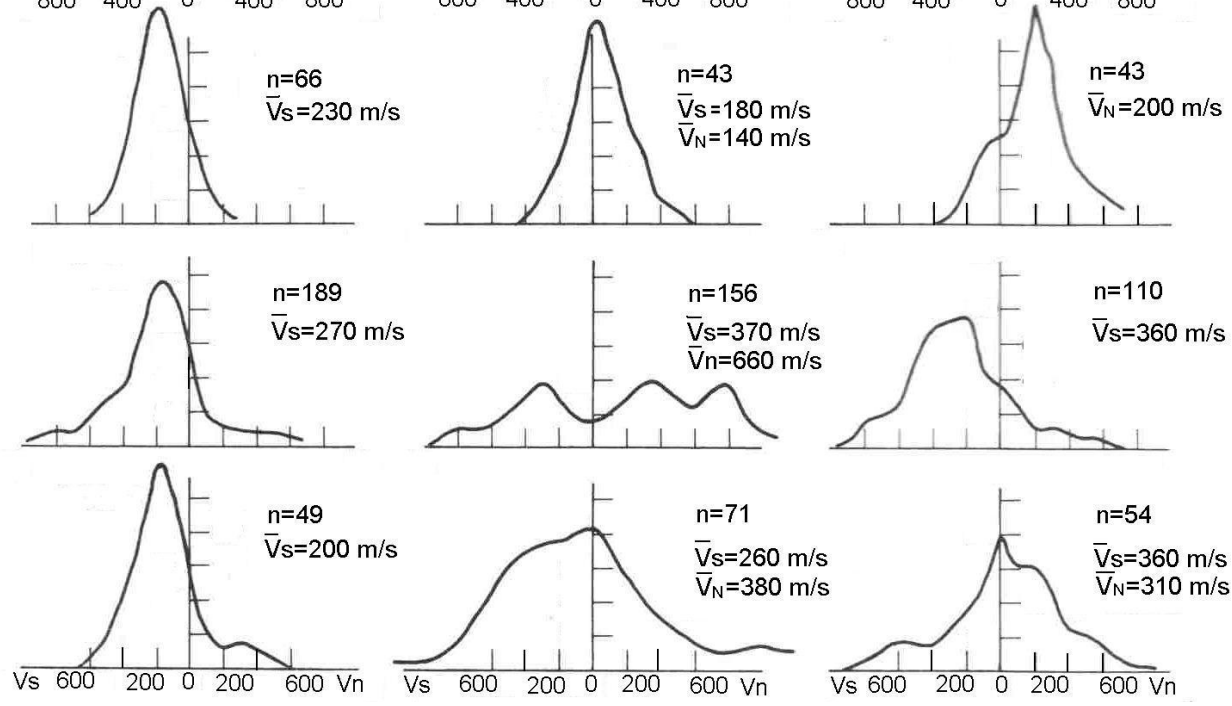

Figure 25. Distribution of meridional velocities of individual auroral forms in the daytime (a), evening (b), midnight (c), and morning sectors (d) of the auroral oval for different substorm phases (Vorobjev et al., 1976b). $V_{\mathrm{S}}$ and $V_{\mathrm{N}}$ represent the mean values of southward and northward arc velocities, respectively, and $n$ is the number of events.

These auroral forms are now widely referred to as "auroral streamers" (Sergeev et al., 1999) and are explained in terms of bursty bulk flows (BBFs) in the plasma sheet (see, e.g. Sergeev et al., 2004).

\subsection{Fine structure of substorms}

In Sect. 7.2 the large-scale activation of auroras during substorms was described, which comprises a considerable longitudinal sector on the nightside. But the detailed comparison of magnetic fluctuations observed in the magnetospheric tail with magnetic disturbances at Earth's surface during substorms has shown that only a longitudinally confined sector of the magnetotail is affected during any given quasiperiodic intensification of a substorm. Rostoker and Camide (1971) suggested that the magnetotail's magnetic field configuration may become even more tail-like, since a confined portion of the tail field is collapsing to a more dipolar configuration during periods of substorm activity intensification. From a statistical study of $500-1000 \mathrm{keV}$ electrons at synchronous orbit, Lezniak and Winckler (1970) concluded that near local midnight there exists a "fault line", west of which substorms are accompanied by geomagnetic field lines inflation and east of which they are accompanied by collapse. The collapse is an inward convective surge of field lines with an average convective velocity estimated at $0.5 R_{\mathrm{e}} \mathrm{min}^{-1}$.
Substorm-associated geomagnetic field line collapses on the nightside of the magnetosphere are always accompanied by $50-150 \mathrm{keV}$ electron accelerations. A collapse occurs in the local time interval of 22:00-06:00 MLT with a typical longitudinal extent of the collapsing region estimated to be about $40^{\circ}$.

With satellite observations in the 1970s and 1980s it was difficult to assemble large-scale pictures of the above mentioned phenomena, because the satellites provided information about the corpuscular precipitations and the auroras only in a limited region around their actual position and for narrow time intervals. However, for the recognition of fine structures in the substorm development it was necessary to use simultaneous information from a sufficiently extended region of longitudes. The azimuthal confinement of the disturbance region in the magnetosphere and the quasi-periodic character of their appearance should impress the peculiarities of substorm development in the auroras, which could be traced with a sufficiently dense network of all-sky cameras in longitudinal direction as it was deployed during the IGY (1957-1958). Vorobjev and Rezhenov (1975) considered in detail the development of five auroral substorms.

Figures 26 and 27 show as an example the auroral dynamics during a substorm on 6 December 1958. Figure 26 illustrates the development of the auroral substorm as observed with all-sky cameras from the stations Dixon Island $\left(\Phi^{\prime}=\right.$ 


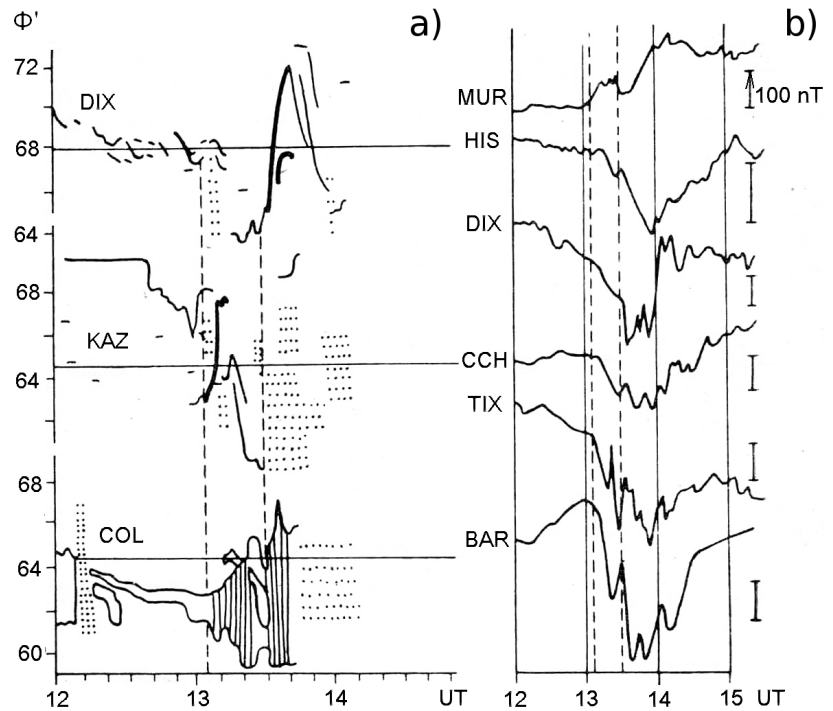

Figure 26. (a) Development of the auroral substorm on 6 December 1958 from Dixon Island, Kazachye, and College ascafilms. (b) Magnetic field $\mathrm{H}$ component records from Murmansk, Heiss Island, Dixon Island, Cape Chelyuskin, Tixie Bay, and Point Barrow (Vorobjev and Rezhenov, 1975).

67.9 $9^{\circ}$, Kazachye $\left(\Phi^{\prime}=64.4^{\circ}\right)$, and College $\left(\Phi^{\prime}=64.9^{\circ}\right)$. The locations of auroras along the magnetic meridian of the stations were determined every minute with the assumption that the luminous height was $110 \mathrm{~km}$. The solid lines represent the position and continuity of discrete auroral forms, while the dots indicate the approximate location of diffuse auroras. The luminous regions (hatched vertically) are shown for College (COL). The $\mathrm{H}$ component of magnetic records from several auroral zone stations are shown on the righthand side of Fig. 26.

Figure 27 shows aurora distribution maps for the same substorm as in Fig. 26 in coordinates of corrected geomagnetic latitude (CGL) versus MLT. The position of the auroral oval is notified there for a medium level of magnetic activity $Q=4$ (Feldstein and Starkov, 1967a) as well as the position of all stations used. The field of view of the cameras is shown by circles with a radius of about $500 \mathrm{~km}$.

An auroral substorm occurred at about 13:05 UT with a fairly well-defined auroral expansion in College and Kazachye, preceded by some magnetic activity. About 20-40 min before the substorm onset, an increase of the equatorward drift of the auroral forms was observed at these stations. The position of discrete forms prior to the substorm onset is shown in Fig. 27 at 13:00 UT. The poleward expansion of the nighttime auroral forms appeared shortly after 13:05 UT and coincided well with the sharp increase of the magnetic disturbances in the $\mathrm{H}$ component. At this time the discrete forms over the evening sector near Dixon Island drifted generally equatorward inside the statistical auroral oval. The position of the active aurora region is shown as a hatched area in Fig. 27 at 13:15 UT. The westward edge of the active aurora region is localised at about 20:00-21:00 MLT and denoted by a dashed line.

The recovery phase in the nighttime sector and a large equatorward shift of the evening-side auroras occurred simultaneously about $15 \mathrm{~min}$ after the substorm onset. A rapid southward shift of the aurora was observed in the regions over the stations Kazachye and Dixon Island. A recovery of the magnetic $\mathrm{H}$ component is observed at the station Point Barrow. The auroral position at the end of the recovery phase is shown in Fig. 27 at 13:25 UT.

At 13:30 UT, i.e. $25 \mathrm{~min}$ after the substorm onset, the arc south of Dixon Island suddenly flashes up and starts to move rapidly poleward. Simultaneously the auroral luminosity above Kazachye and College intensifies. The area of the auroral luminosity broadens both poleward and equatorward. This auroral intensification coincides with a new, abrupt reduction in the $\mathrm{H}$ component of the magnetic field as observed at Point Barrow. The location of the newly formed active aurora region is shown in Fig. 27 at 13:40 UT. The western edge of this region is situated between the stations Dixon Island and Heiss Island, i.e. at about 17:00 MLT. The aurora has not been recorded neither in Heiss Island at the very centre of the statistical oval nor in the station Murmansk southward of it.

In this way the substorm considered consists of two activations (microsubstorms), in the course of which the active auroral region shifts abruptly westward by about $30^{\circ}$. Out of five substorms investigated by Vorobjev and Rezhenov (1975), two activations were observed in three of them, three and four activations in each one of the others.

Generalising these observations, Vorobjev and Rezhenov (1975) proposed a schematic for the development of auroral active regions as shown in Fig. 28. The model variation of the magnetic field $\mathrm{H}$ component at stations in the midnight sector of the auroral zone is shown in the bottom part of this figure. Abrupt reductions of the magnetic field are labelled as the moments $T_{0}, T_{1}$, and $T_{2}$. These reductions in the $\mathrm{H}$ component correspond to the sequence of activations in longitudinally limited parts of the oval. The character of the changes during each of these activations agrees generally with the conception of auroral substorm development by Akasofu (1964a). The activity starts to develop at the time $T_{0}$ in the midnight sector of the oval. In the course of the activation, the auroral bulge develops, the western edge of which is located within 20:00-22:00 MLT. Subsequent auroral activations (the moments $T_{1}$ and $T_{2}$ ) are accompanied with jump-like shifts of the western edge of the active aurora region by $30-50^{\circ}$ in longitude toward earlier local times up to $16: 00$ MLT.

The western edge of developing auroral bulges is sufficiently well formed. During the expansive phase one observes only a small shift of it toward the evening side without any westward travelling surge formation. During the recovery phase, however, which usually starts $20-30 \mathrm{~min}$ after the moments $T_{0}, T_{1}$, and $T_{2}$, the auroral bulges decrease and their 

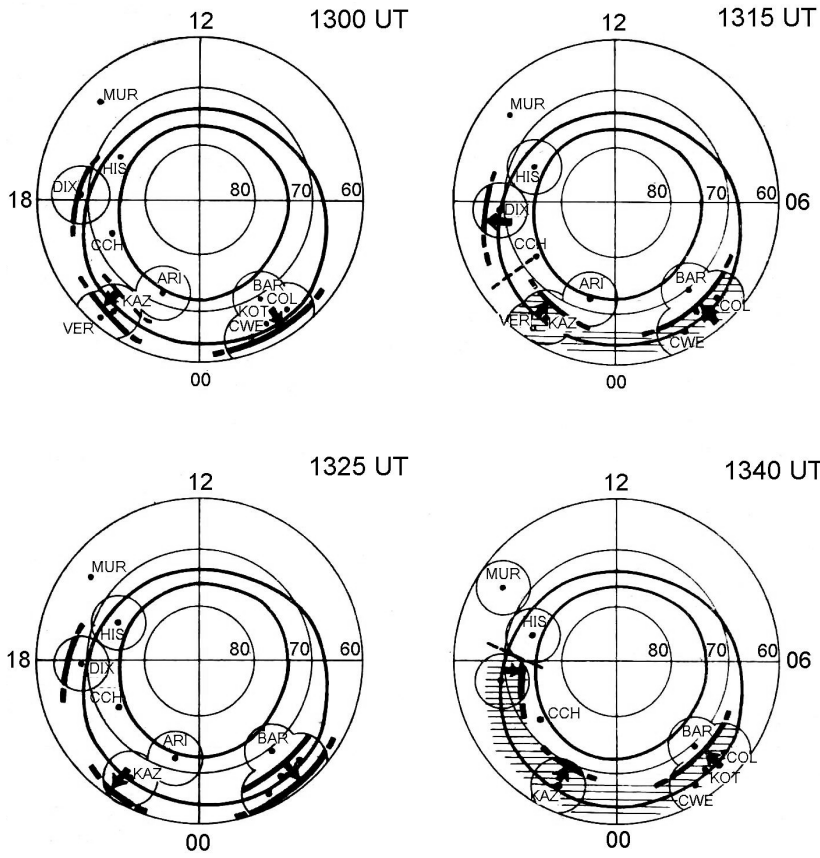

Figure 27. Auroral distribution maps during the substorm on 6 December 1958. Boundaries of the $Q=4$ statistical auroral oval are shown by solid lines (Vorobjev and Rezhenov, 1975).

western edge shifts eastward. Often the recovery is accompanied by an emergence of wrinkles in the auroras which move eastward.

About 20-60 min prior to $T_{0}$, in the midnight sector of the oval, there starts either an intense equatorward movement of the arcs or a rapid shift of the southern auroral border toward the Equator. Such movements are typical for the growth phase of substorms as reported by Ivliev et al. (1970), Feldstein and Starkov (1970a), Starkov and Feldstein (1971a), and Snyder and Akasofu (1972). It might be interesting to note that such phenomena are also observed prior to the moments $T_{1}$ and $T_{2}$ in those regions of the oval, where the activity subsequently develops.

Such a scheme presumes a series of jump-like relocations of the westward electrojet in westward direction (or the formation of new ones) and a corresponding shift of the activation region in the magnetospheric tail, which would agree with the results of Rostoker and Camide (1971). Kisabeth and Rostoker (1971) and Rostoker (1974) showed the development of two parallel electrojets, where the equatorward one developed first, while the poleward one appeared 15-20 min later and was also displaced westward.

Sergeev and Yahnin (1979a, b) have used simultaneous allsky camera observations from eastern Siberia, Alaska and Canada to examine in detail the substorm expansive phase signatures. They discovered that the development of the auroral expansion consists of a series of localised expansions (usually $\sim 40^{\circ}$ in longitude), which unfold in different MLT
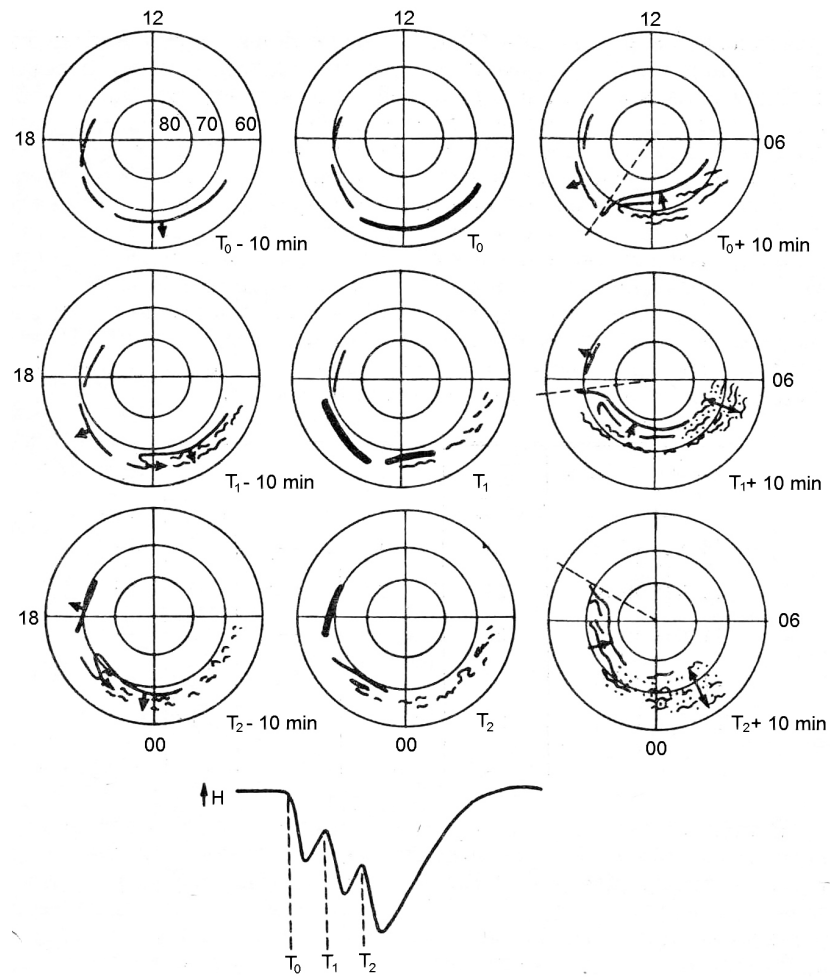

Figure 28. The summary scheme of an auroral substorm development and a magnetic field $\mathrm{H}$ component model (Vorobjev and Rezhenov, 1975).

sectors. The authors demonstrated that a subsequent activation has to occur westward of the preceding, but a jump-like broadening of the active aurora region in the eastward direction is likewise possible. Each of these activations possesses all attributes of a substorm expansion phase: (a) fast poleward expansion of the auroral bulge; and (b) activation of the westward electrojet; (c) increase of the $\mathrm{H}$ component of the magnetic field at lower latitudes of the corresponding MLT sector; (d) excitation of Pi2 pulsations (Pytte et al., 1976a; Sergeev, 1977a; Sergeev and Yahnin, 1979b), which led to the term "microsubstorm" for these activations.

Microstructures within substorms are not a rarity. In fact, the majority of disturbances which can be classified as a substorm reveal microstructures when they are investigated in detail. The existence of microsubstorms has been reliably confirmed by observations with magnetospheric satellites. Sergeev (1974, 1977b) and Pytte et al. (1976b) showed that typical signatures of the substorm expansive phase are localised in the magnetosphere along the $Y$ direction and series of activations occurred in different intervals of $\Delta Y$. These authors noted that it is typical for the nightside magnetosphere that "butterfly-like" pitch-angle distributions of electrons change to isotropic ones just after substorm and microsubstorm onsets. The period of isotropisation lasts thereby usually 5-10 min. Later on up to the beginning of a following 
microsubstorm, a recovery to the quiet-time pitch-angle distribution was observed. Thus, it is possible to claim that typical substorm expansive phases last about 5-10 min, including a fast poleward expansion of bright auroras and an insignificant growth of field-aligned current intensity. Furthermore, Sergeev (1974) emphasised that in such events the definition of substorm phases for the whole magnetosphere is ambiguous, because features that are typical for different phases can occur in different parts of the magnetosphere simultaneously.

\section{Structure and dynamics of auroras during intervals of steady magnetospheric convection}

The relations between plasma convection and the position of discrete auroral forms are quite complicated. The challenges are related to difficulties in determining the azimuthal movement of elongated auroral forms and their velocity according to photographic images of the S-180 camera and to the MLT dependence of the auroral motion's characteristics. The direction and magnitude of the auroral movement is most concisely determined in the daytime and the nighttime sectors, where the discrete forms move mainly in meridional direction. Figure 29 shows a scheme of ionospheric convection, which was deduced from observations of discrete form motions during creation (on the left side) and recovery phases (right side) (Vorobjev et al., 1976b). This scheme was obtained under the assumption that the direction of auroral motion coincides with the direction of the convection. The motion is indicated by arrows within the corresponding MLT sectors. Usually additional independent data of the ionospheric convection are used for the determination of the convection's direction and its relation to the auroras in the dawn and dusk sectors. In this way Sandholt and Farrugia (2007) consulted measurements of ionospheric plasma drift by SuperDARN radars, while Feldstein et al. (2001) considered electric field measurements on board of the Viking satellite. The auroral oval and the diffuse luminosity equatorward of the oval reside in the morning sector at latitudes with sunward convection, while antisunward convection comprises the latitudes between the oval and the polar cap (the lowlatitude boundary layer). In the evening sector, however, the auroral oval is located at latitudes with antisunward convection and the diffuse luminosity equatorward of the oval span latitudes of sunward convection.

Apart from intervals of magnetic storms and substorms, a considerable magnetic activity is observed during periods of so-called steady magnetospheric convection (SMC). The existence of protracted SMC periods, which develop under the influence of a prolonged southward $B_{z}^{\mathbb{M F}}$ component, was first described by Sergeev (1977a) and Pytte et al. (1978). During such periods one observes significant disturbances of the Dst index, strong and fluctuating disturbances at auroral latitudes, and a two-vortex current system in the polar region. However, analysis of both ground-based and spacecraft
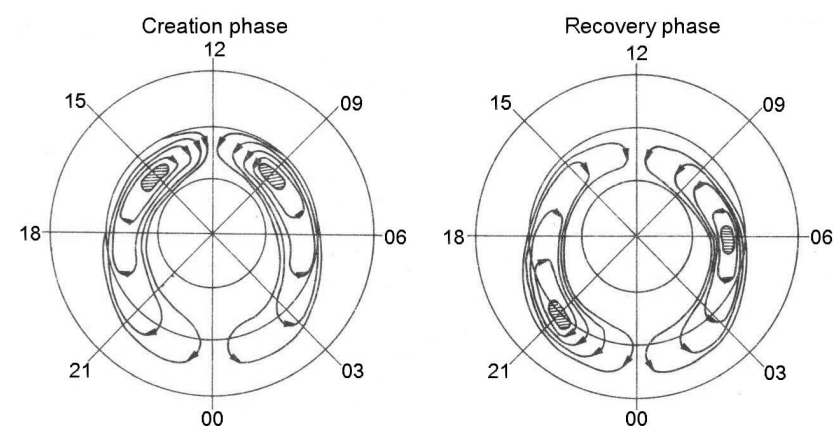

Figure 29. Schemes of ionospheric convective motion during the creation and recovery phase of substorms obtained from data concerning the motions of discrete auroral forms (Vorobjev et al., 1976b).

observations indicate the absence of substorm signatures during many hours of high geomagnetic activity that testified the steady state of the magnetotail and the ionosphere for many (sometimes more than 10) hours. The terminology is yet ambiguous and such events have been referred to as "steady convection activity" (Sergeev, 1977a), "convection bays" (Pytte et al., 1978), or "steady magnetospheric convection (SMC)" (Yahnin et al., 1994).

Structure and dynamics of nighttime auroras during SMC events were investigated for the first time by Sergeev and Vorobjev (1979). They examined in detail the behaviour of auroras on 3 December 1973, when two intervals of SMC were observed. The characteristics of the interplanetary medium (King, 1977) and the indices AU and AL of the magnetic activity in the auroral region (Allen et al., 1975) are shown in Fig. 30. After about 07:00 UT, the $B_{z}^{\mathrm{IMF}}$ component turned southward and stayed negative till the end of the day. The solar wind velocity remained throughout the whole period at a speed of $280 \mathrm{~km} \mathrm{~s}^{-1}$. Two intervals could be clearly identified, 10:00-16:00 UT and 18:00-22:00 UT, during each of which the magnetic disturbances in the auroral zone stayed at about the same level while there were no significant changes of the interplanetary medium parameters. The absence of strong IMF fluctuations is shown by the small values of variance $(\sigma)$ for the IMF vector magnitude compared with the values of the $B_{z}^{\mathbb{M}}{ }^{\mathbb{M F}}$ component. Vertical dashed lines in Fig. 30 mark the moments of overflight of two DMSP satellites over the northern polar region; the trajectory of one of them crossed the oval in the sector of 21:0022:00 MLT, the other shortly after midnight. The first interval of 10:00-16:00 UT deserves closer attention, because it is characterised by particularly stable IMF and solar wind parameters.

The vertical lines in Fig. 31 indicate the initiation moments of Pi2 pulsation trains at midlatitudes. In these moments one observes an intensification of the westward electrojet at auroral zone stations close to midnight and disturbances of the $\mathrm{D}$ and $\mathrm{H}$ magnetic field components at midlatitudes (stations: 


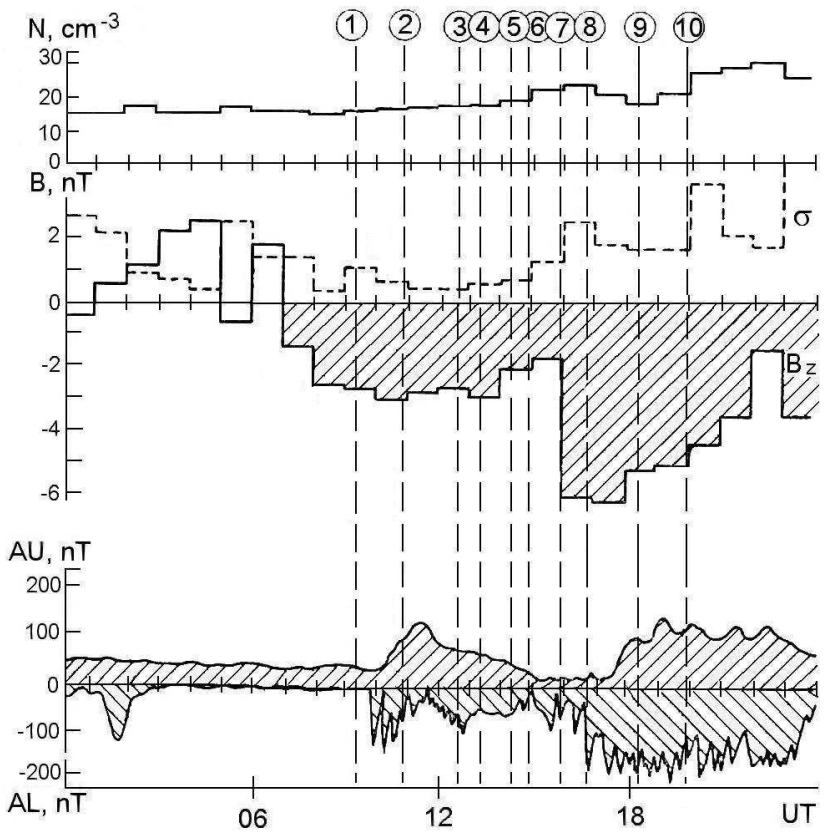

Figure 30. Solar wind plasma density and IMF parameters (two top panels) and variations of the AU and AL magnetic indices on 3 December 1973. Vertical dashed lines mark the moments of overflight of two DMSP satellites over the northern polar region (Sergeev and Vorobjev, 1979).

Magadan, $\Phi=51.0^{\circ}$; Irkutsk, $\Phi=41.0^{\circ}$; Lvov, $\Phi=47.8^{\circ}$ ), which are characteristic for substorms. During the intervals of interest here, neither Pi2 oscillations nor characteristic magnetic substorm variations at midlatitudes were observed, and therefore these intervals can be identified with periods of SMC.

The satellite images are unfortunately of low quality, so the structure of auroras during the first SMC interval is only schematically shown in Fig. 32. Discrete forms (heavy lines) and the diffuse luminosity (vertical dashed lines) are displayed in more detail. Frame (1) corresponds to the substorm creation phase and frame (2) is characteristic for the substorm expansive phase: the diffuse aurora intensified, a small auroral bulge formed up at the western edge from which an auroral surge became visible. Frames (3), (5), and (7) correspond to the period of SMC. The apparent auroral characteristics there support the identification of the selected interval as period of SMC, because the structure of the aurora, the position of the surge ( $\sim 21: 00 \mathrm{MLT})$, the location of the auroral boundaries, and their extent in latitude $\left(5-6^{\circ}\right)$ persist constantly for several hours.

Additionally, frames (3), (5), and (7) show the presence of small-scale variations in the auroras. Changes in the structure of the auroral bulge and its western edge can be noticed from orbit to orbit, the brightness is inhomogeneous and intensified in regions where a small-scale spiral structure can be noticed. All this proves the existence of complicated dy-

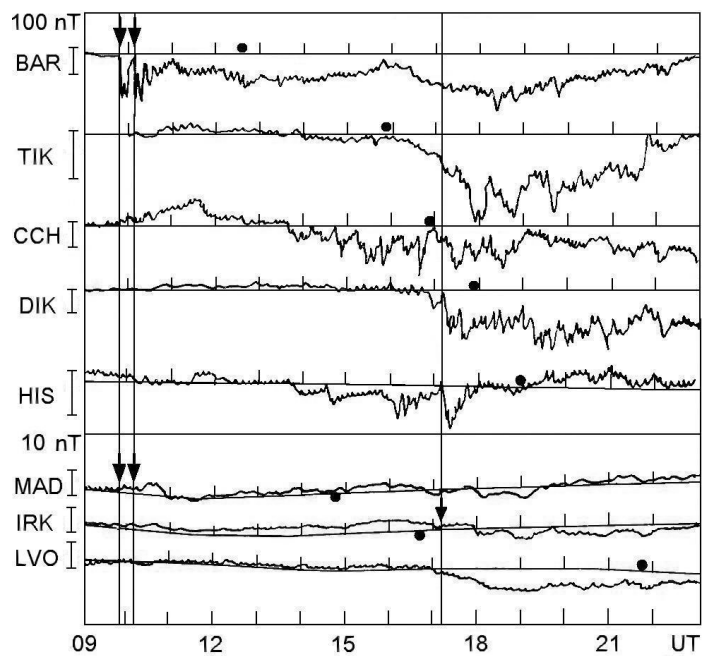

Figure 31. Magnetograms of auroral (from top to bottom: Point Barrow, Tixie Bay, Cape Chelyuskin, Dixon Island, Heiss Island) and midlatitude stations (Magadan, Irkutsk, Lvov) on 3 December 1973. The vertical lines indicate the initiation moments of Pi2 pulsation trains in midlatitudes (Sergeev and Vorobjev, 1979).

namic variations in the structure of the precipitations, without touching, however, its main large-scale characteristics.

Figure 33a presents data of ground-based all-sky cameras for the second SMC interval. It shows the position of the auroral forms in the meridian of the observatory Dixon Island $\left(\Phi^{\prime}=67.9^{\circ}\right)$, assuming a height of $110 \mathrm{~km}$ for the aurora. The figure shows that discrete auroral forms exist at the poleward edge of the auroral bulge during the hours around local midnight. Sometimes their intensity can somehow diminish so that their existence becomes hard to detect in the ascafilms. After a certain time, however, the arcs reappear in the same region. Often one observes discrete forms within the auroral bulge, which move equatorward.

An intense luminosity exists during the whole period (18:00-22:00 UT) at the southern horizon of the observations, which can be identified in comparison with the spacecraft frames as a region of diffuse aurora. Between the discrete auroral forms toward the north and the diffuse aurora toward the south, a dark gap is clearly seen, which fills up from time to time with irregular structures. Such an auroral behaviour is characteristic for the whole second interval of SMC.

Figure $33 \mathrm{~b}$ presents the auroral behaviour at the meridian of the observatory Loparskaya $\left(\Phi^{\prime}=64.8^{\circ}\right)$ during the interval 18:00-24:00 UT. These data allow one to consider the similar situation in the region of the diffuse aurora. Up to 19:55 UT the camera worked at a simplified regime (one picture per five minutes) as indicated by the hatched area. The most significant auroral intensifications, which occurred around 22:35 and 22:10 UT, were related to the passage of eastward propagating $\Omega$ structures above the station. Similar 

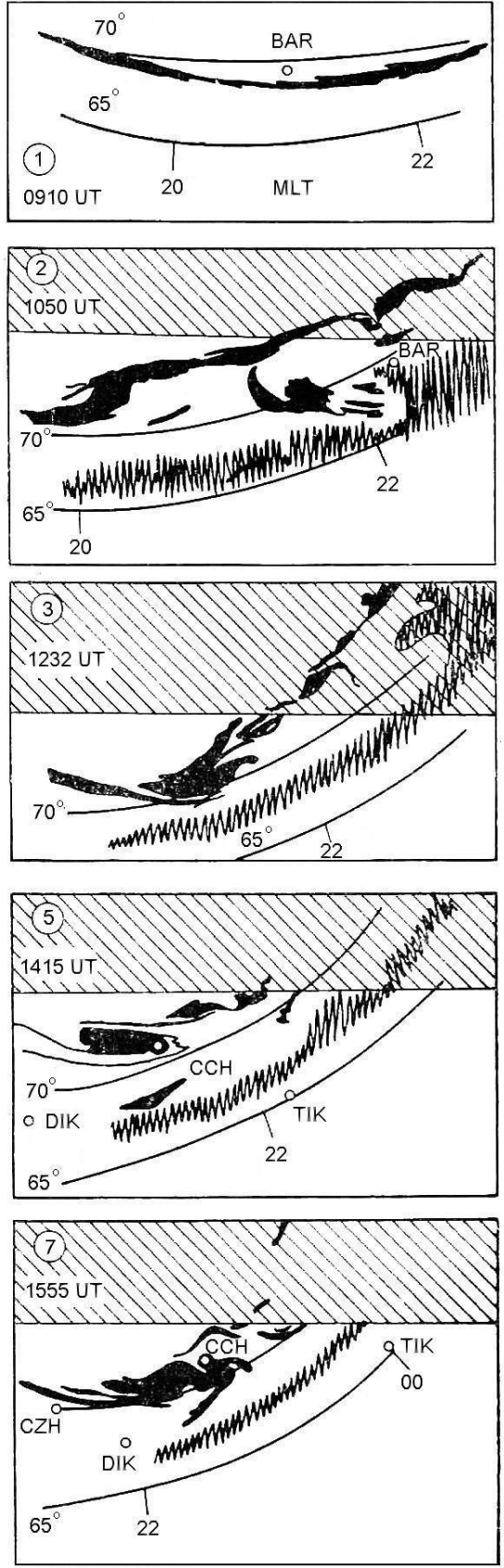

Figure 32. Schematic auroral display from DMSP spacecraft frames in the coordinates of corrected geomagnetic latitude-MLT. Locations of magnetic observatories are marked by dots. The number of frame is indicated by a digit in the ring (Sergeev and Vorobjev, 1979).

structures appeared also at satellite photographs, so that their presence seems to be a characteristic feature during periods of SMC.

The detailed analysis of the two SMC intervals on 3 December 1973, and a number of other similar examples have enabled Sergeev and Vorobjev (1979) to construct a scheme

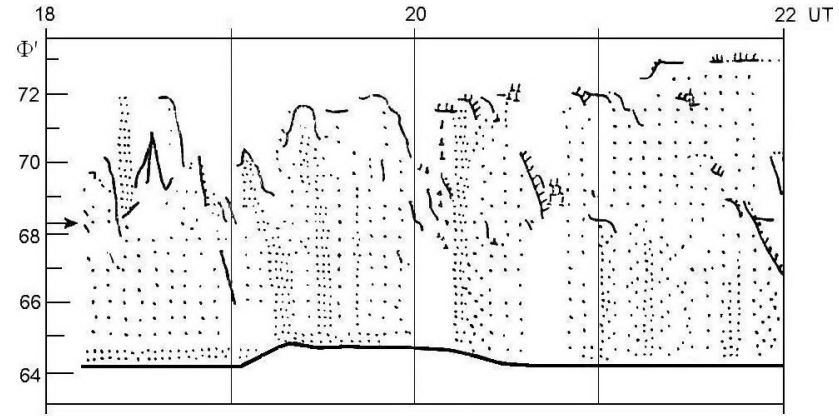

Figure 33a. Auroral dynamics on the meridian of Dixon Island observatory on 3 December 1973. Positions of homogeneous auroral forms (arcs or bands) are shown by solid lines; that of rayed arcs by herringbones; diffuse aurorae by dots (Sergeev and Vorobjev, 1979).

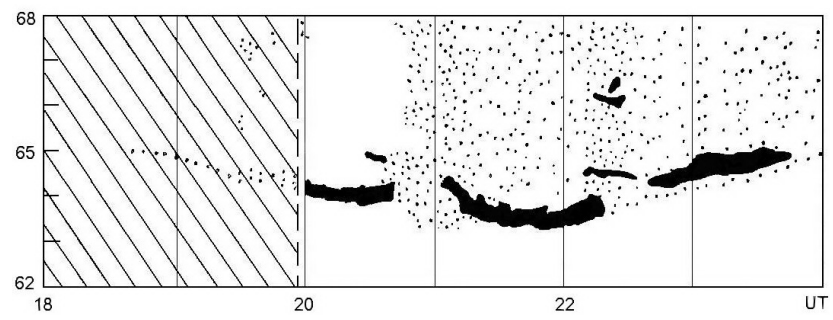

Figure 33b. Auroral dynamics on the meridian of Loparskaya on 3 December 1973, in the same manner as Fig. 33a, but for a $2 \mathrm{~h}$ longer interval.

of the auroral structure during periods of SMC, which is presented in Fig. 34a. The auroral display consists of diffuse auroras at the equatorward side of the precipitation region and discrete forms poleward of the diffuse luminosity. The discrete arcs are regular and intense in the evening sector. One or more discrete arcs can be observed. On the nightside one observes an auroral bulge, where its western edge appears as a large-scale surge. At the poleward edge of the auroral bulge region the arcs appear irregular both in structure and intensity, where the structures before local midnight are systematically brighter than after midnight. Usually one observes between the discrete and the diffuse auroral regions on the nightside a dark gap of several degrees of geomagnetic latitude. From time to time this gap fills up with diffuse luminosity and/or irregular structures, which usually move equatorward. The poleward border of the diffuse aurora is irregular and $\Omega$ structures can often be noticed on it, which are drifting eastward. Layered structures of diffuse auroras are observed in the morning sector and their intensity there is stronger than in the evening sector.

All these characteristic features, as can be easily seen, correspond closely to auroral structures at the maximum of the substorm expansive phase. The position of the auroral bulge, however, appears to be stationary in case of SMC periods. This means that the large-scale characteristics of 

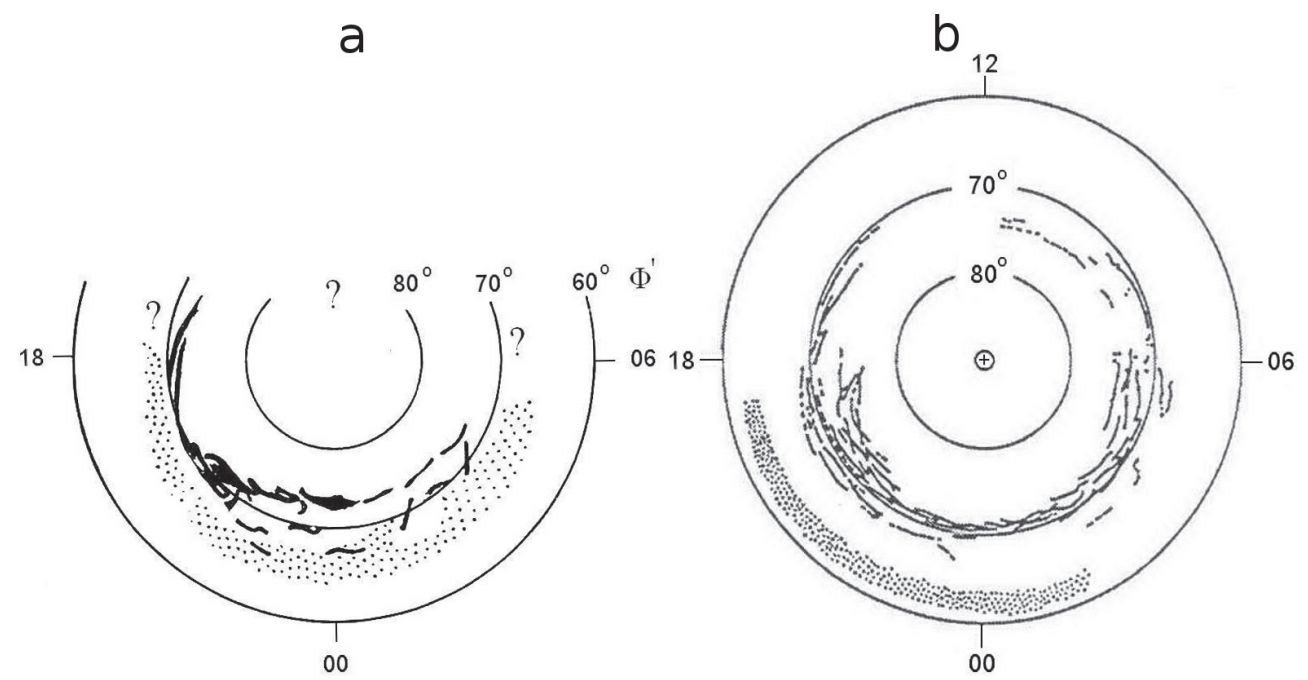

Figure 34. Distribution of discrete and diffuse auroras during the SMC periods. (a) Nighttime auroral pattern after Sergeev and Vorobjev (1979); (b) summary of the different auroral forms observed by all-sky cameras on 24 November 1981 (Yahnin et al., 1994).

precipitations during SMC periods are mainly caused by magnetospheric structures and not by dynamic processes, which develop during magnetospheric substorms.

Despirak et al. (1994) used the prolonged, about $10 \mathrm{~h}$, SMC interval on 24 November 1981 to examine the configuration and the location of the cusp-like area from dayside NOAA spacecraft observations. They found the cusp-like precipitation at $72-75^{\circ}$ invariant latitudes between 10:00 and 12:00 MLT. Ground-based magnetic and radar data showed that this region coincides with the dayside convection throat. All-sky camera observations demonstrated that short-lived, sporadic, discrete auroral arcs and long-lived stable discrete arcs bound the cusp region from the pre-noon and from the after-noon, correspondingly. The cusp region coincided well with the gap in the global distribution of discrete auroras (Vorobjev and Rezhenov, 1982). It is very interesting that the cusp region was situated poleward of the highlatitude boundary of the UVI emission auroral belt observed by the DE-1 spacecraft imager in the $123-160 \mathrm{~nm}$ wavelength range.

A global auroral display was examined by Yahnin et al. (1994) during the SMC interval on 24 November 1981, with the duration of more than $10 \mathrm{~h}$ under the steady southward IMF field without any distinct substorm signatures. The authors used all-sky camera films from the observatories which are situated in Greenland, Spitzbergen, and Alaska and recorded auroral behaviour in the dark Northern Hemisphere. Figure 34b provides a summary of all auroral forms observed by all available all-sky cameras. The cameras were distributed in longitude, and different stations scanned the same MLT sector at different universal times. In order not to overload the figure, only the auroral structures seen at the first minute of every UT hour were actually plotted. Horizontal positions of auroral forms seen on the original all-sky films were mapped to the $110 \mathrm{~km}$ height level and then plotted in CGL-MLT coordinates. Approximate positions of the diffuse aurora are also shown schematically by dots. The typical distributions of the auroras seen by the different cameras are basically similar.

As shown in Fig. 34b, the nightside auroras were confined within two distinct ranges of latitudes: discrete arcs at latitudes $68-75^{\circ} \mathrm{CGL}$, and diffuse auroras between 60 and $65^{\circ}$ CGL. The discrete auroras were highly variable, showing short-time intensifications and decays, and the formation of folds and loops. These active auroras were bordered in the evening sector by a surge-like structure. In the midnight sector, at latitudes between the domains of discrete and diffuse auroras, relatively weak structures or active arcs typically drifted either equatorward from the poleward domain of the discrete structures or in the zonal direction. The poleward edge of the diffuse aurora was sometimes disturbed by torchlike structures. Rather stable and bright discrete arcs were observed in the afternoon sector, while in the morning sector short-lived multiple discrete arcs intermittently appeared up to the dayside, where auroral forms such as the rayed arcs and rays were observed as well.

The main characteristics of the auroral distribution presented in Fig. 34b agree well with the description of the auroral pattern given by Sergeev and Vorobjev (1979). The only difference between them concerns the surge-like structure at the poleward boundary of the auroral oval. While this is located at about 21:00 MLT according to Sergeev and Vorobjev (1979), it is found at 18:00-19:00 MLT for the event in Fig. 34b. This difference in longitude can be attributed to the effect of the $B_{y}^{\mathrm{IMF}}$ component.

The isotropic boundaries of the $>30 \mathrm{keV}$ protons and electrons were found by Yahnin et al. (1994) close to each other, separating regions of discrete and diffuse precipitation. 
This suggests that these precipitation types originate from tail-like and dipole-like field lines, respectively.

\section{Nighttime diffuse luminosity equatorward of the auroral oval}

The nighttime aurora is characterised by a quite complicated structure, which includes not only discrete forms at latitudes of the auroral oval. Thus, Akasofu (1974b) identified three different types of auroras based on both airborne (Whalen et al., 1971) and satellite observations (Lui and Anger, 1973) at auroral latitudes: the discrete, the continuous, and the diffuse luminosity. The latter two types represent the diffuse forms: continuous along the auroral oval and diffuse along the quasi-circular belt. Continuous luminosity proves to be the background one, in which the discrete auroral forms are embedded. A belt of diffuse auroras encompasses Earth along a parallel of corrected geomagnetic latitudes, coinciding in the midnight sector with the auroral oval. Such an identification appears not to be exhaustive. One has to include yet the diffuse aurora equatorward of the auroral oval, which is the subject of this section.

According to the International Auroral Atlas (1963), the diffuse auroral forms can have the appearance of spots with fuzzy boundaries or hazes. Photometric or spectrographic observations on board polar orbiting spacecrafts and on ground are usually used for the study of diffuse aurora. The $630.0 \mathrm{~nm}$ emission dominates in the spectral composition of diffuse auroras during nighttime hours. Its intensity exceeds that of the $557.7 \mathrm{~nm}$ emission by a factor of $\sim 4$. Based on the methodology of Rees and Luckey (1974), this indicates an average energy of the precipitating electrons of $E \sim 0.1-0.2 \mathrm{keV}$, which corresponds to an altitude of the luminescent layer near $\sim 200 \mathrm{~km}$. The intensity of the diffuse aurora amounts from several tens of Rayleighs to a few kilo-Rayleighs; it decreases with decreasing latitude and increases with increasing magnetic activity (Akasofu and Chapman, 1962; Krasovsky, 1967). Intense diffuse luminosity of up to several kilo-Rayleighs is observed even at midlatitudes during geomagnetic storm periods, accompanying the storm development (Chapman, 1957). The emission of the ING $\mathrm{N}_{2}^{+}$band, which is characteristic for nighttime, discrete auroral forms, appears as a very faint diffuse luminosity (Alekseev et al., 1975). This indicates lower energy values of the precipitating electrons in diffuse auroras compared with discrete forms. Visual observations of the diffuse auroras are rare due to its relatively weak intensity, the hardly distinctive contrast of luminosity at the firmament, and the low sensitivity of the human eye in the red spectral range of the doublet (OI) 630.0 $636.4 \mathrm{~nm}$ (Krasovsky, 1967).

The existence of a region of diffuse subvisual luminosity equatorward of the auroral oval in the nighttime sector was deduced from ground-based observations (Reid and Rees, 1961; Galperin, 1963; Sandford, 1964, 1968; Eather, 1967;
Wiens and Vallance, 1969; Evlashin, 1971). Parallactic observations with scanning photometers along the geographic meridian of $\sim 129^{\circ} \mathrm{E}$ (from Yakutsk, $\Phi^{\prime}=55.8^{\circ}$, to Tixie Bay, $\Phi^{\prime}=65.2^{\circ}$ ) have revealed, that the diffuse background luminosity of $630.0 \mathrm{~nm}$ emissions span over an altitude range of 140-300 km (Alekseev et al., 1975). Detailed morphological studies of diffuse auroras were done based on ISIS-2 satellite observations (Lui and Anger, 1973; Lui et al., 1973, 1975, 1977). These authors confirmed the existence of the diffuse luminosity, which was described before by groundbased spectral measurements (Evlashin, 1961; Rees et al., 1961). The ISIS-2 spectral data (Lui and Anger, 1973; Wallis et al., 1976; Lui et al., 1977) indicate that the major part of the observed intensity of diffuse luminosity is provided by a low-energy (0.1-1.0 keV) electron precipitation. Mende and Eather (1976) have also concluded that the major fraction of the luminosity in the diffuse auroral region is associated with electron precipitation.

The frames of auroral luminosity obtained from DMSP (Nagata et al., 1975; Pike and Dandekar, 1979; Nakai and Kamide, 1983) and ISIS-2 satellites (Lui et al., 1975), as well as from ground-based photometric observations (Slater et al., 1980; Alekseev et al., 1980), were used to obtain quantitative relations between the position of the equatorward diffuse auroral boundary (DAB) and the magnetic activity. Slater et al. (1980) deduced from ground-based observations of $630.0 \mathrm{~nm}$ emissions near midnight the dependence of DAB's geomagnetic latitude on the $K p$ index:

$\Phi_{\text {DAB }}^{\prime}=67.46^{\circ}-2.04^{\circ} \mathrm{Kp}$.

At auroral and subauroral latitudes, there exists a fairly broad belt of subvisual atmospheric luminosity, which has a different spectral composition than the diffuse electron luminosity. Spectrometer observations revealed the existence of hydrogen emissions of the Balmer series (Balmer emissions) (Vegard, 1939, 1950; Meinel, 1950; Gartlein, 1950). This luminescence was named proton aurora and has been broadly investigated with the materials of the IGY (Rees et al., 1961; Evlashin, 1961; Omholt et al., 1962; Galperin, 1959, 1963; Eather, 1967; Sandford, 1968; Lui et al., 1973). The planetary distribution of the proton aurora constitutes a belt which is situated at latitudes of the auroral oval and equatorward of it (Eather, 1967; Rees and Benedict, 1970; Evlashin et al., 1972). The relationship between proton and electron emissions have been investigated in detail by Oguti $(1973,1975)$ and Fukunishi (1973, 1975).

Frank and Ackerson (1971), Ackerson and Frank (1972), and, more in detail, Frank et al. (1976) by using Injun 5 satellite data showed that a region of non-structured precipitations with lower-energy electrons exists equatorward of the structured precipitations in the nighttime sector, which should cause the diffuse luminosity. The intensity of the precipitating fluxes decreases monotonically from midnight to late-morning and daytime hours, while the electron energy increases. 
The diffuse luminosity has a fairly pronounced lowlatitude boundary in the nighttime sector (Lui and Anger, 1973; Snyder and Akasofu, 1974; Pike and Whalen, 1974), which is closely related to the fluxes of precipitating lowenergy electrons. Therefore, satellite measurements were extensively used to find the position of the equatorward diffuse precipitation boundary (DPB) of such particles (Galperin et al., 1977; Kamide and Winningham, 1977; Gussenhoven et al., 1981; Hardy et al., 1981). The DPB coincides quite closely with the position and variation of the DAB. In the nighttime sector, the DPB is situated near the footprint of the plasmapause at ionospheric heights. The plasmapause represents the shell with a jump-like increase of thermal plasma density. It is the near-Earth boundary of the magnetospheric convection, where the plasma motion from the plasma sheet in the magnetospheric tail brakes toward Earth (Nishida, 1966).

The diffuse auroral belt without any discrete auroral forms adjoins to the auroral oval. Its poleward (high-latitude) boundary constitutes the equatorward boundary of the auroral oval and at the same time it is the high-latitude boundary of the radiation belt $(\mathrm{RB})$ of electrons with energies from a few tens to hundreds of kiloelectronvolts (STB - stable trapping boundary for radiation belt electrons). This STB can be most easily and effectively determined with particle detectors by tracing the disappearance of geomagnetically trapped electrons with $E \sim 25-40 \mathrm{keV}$ (O`Brien, 1963; McDiarmid et al., 1975). Observations of trapped electron distributions revealed that the outer edge of the radiation belt (STB) has an asymmetric form similar to the auroral oval and is nearly collocated with it (O`Brien, 1963; Frank et al., 1964; Akasofu, 1968). This collocation extends to all levels of geomagnetic activity (Feldstein and Starkov, 1970b). The region of diffuse luminosity equatorward of the auroral oval is therefore located more equatorward than the STB. Such a relation between the STB and the diffuse auroral belt was confirmed by Vorobjev et al. (1976a).

Peaks of rapid westward ion drift of up to several kilometres per second are observed at ionospheric altitudes equatorward of the boundary of diffuse auroral emissions. Galperin et al. (1974) were the first who reported strong, polewarddirected electric fields during sunward plasma convection at dusk side subauroral latitudes and called them polarisation jets (PJ). Nowadays this phenomenon is known as sub-auroral polarisation streams (SAPS) (Foster and Burke, 2002). SAPS are located in the region of the ionospheric through. This phenomenon is therefore not accompanied by intense electric currents in the ionosphere.

Feldstein and Galperin (1985) reviewed the morphology and dynamics of the diffuse aurora region, its position and intensity of luminosity, as well as its dependence on various geomagnetic activity indices and plasma precipitations. Galperin and Feldstein (1996) and Feldstein and Galperin (1996) discussed the structure of the auroral luminosity, the boundaries between its various regions, and the relations with the plasma structures of the nighttime magnetosphere. The diffuse aurora region equatorward of the auroral oval is caused by electron precipitation from a region between the central plasma sheet in the magnetospheric tail and the plasmapause (remnant layer). The electrons undergo a drift motion from the plasma sheet toward the Earth and on the morning side. They are accelerated during its drift by the large-scale dawn-dusk electric field and precipitate into the upper atmosphere. The similarity of the spectra at various altitudes follows from a comparison of energy spectra of those electrons, which are transported by convection from the plasma sheet, with those of the diffuse luminosity at the same geomagnetic field lines (Lui et al., 1977; Meng, 1978). The maxima of the energy spectra are identical in intensity at both ends of the field line, and the spectra themselves are characterised by a monotonic descent from a maximum value in accordance with the energy decrease of the particles. The absence of an additional maximum in the spectra shows that there is no acceleration by field-aligned electric fields along the corresponding magnetic field lines.

The intensity of the diffuse aurora increases heavily during the main phase of geomagnetic storms (Akasofu and Chapman, 1962). This became well recorded by all-sky cameras and it was clearly seen through visual observations. With increasing magnetic activity, the diffuse luminosity appears more and more at lower latitudes. During periods of very strong magnetic storms, visual registrations at meteorological stations became predominant. Such observations were used to determine the latitude of the equatorward boundary of the auroral luminosity for various levels of the planetary geomagnetic index $K p$ and for the compilation of visoplots, where the distribution of auroral regions is marked with various symbols. Comprehensive data about visoplots during the IGY were published in Annals of the IGY (1964). Using visoplot data, Zverev et al. (2009) determined the borderline latitude values of the nighttime diffuse luminosity distribution. They applied this to intervals of strong magnetic storms with $D s t<-100 \mathrm{nT}$. The position of the equatorial boundary of the luminosity (DAB) is shown in Fig. 35. The equation for the regression line

$$
\Phi^{\prime}=74.7^{\circ}-12.05^{\circ} \log |D s t|
$$

is shown as a solid line with a regression coefficient of $r=0.7$. For a change of Dst from -100 to $-400 \mathrm{nT}$, the boundary will move equatorward by $\sim 7^{\circ}$. According to the visoplot data, the luminosity is recorded at all latitudes between the DAB and the auroral oval.

It is generally known that the boundary of the auroral oval also moves equatorward during magnetic storm intervals. The amount of the shift is determined by the magnitude of Dst variations. The dots in Fig. 35 show the position of the equatorward boundary of the auroral oval as arranged by Starkov (1993) in dependence on the Dst index according to various researchers. The dashed line shows the regression 


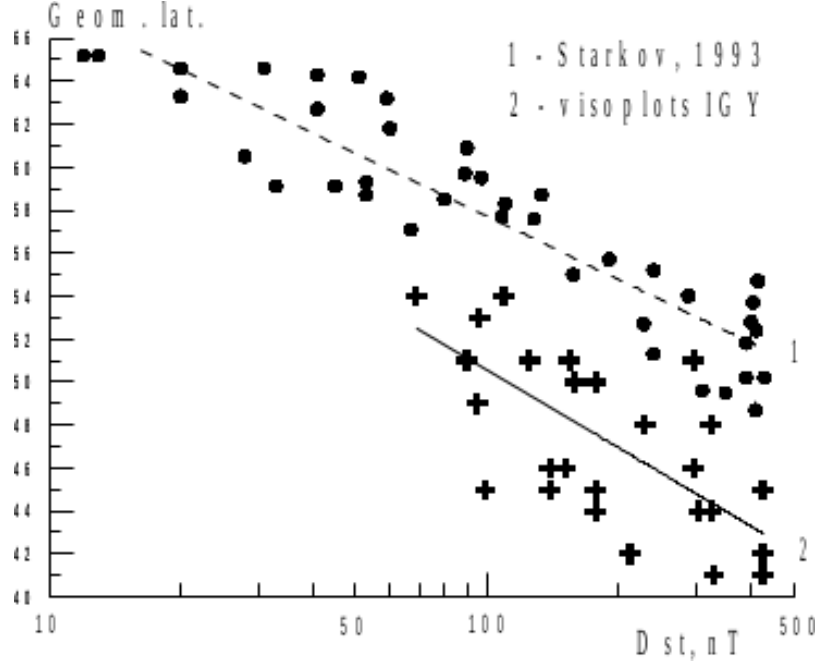

Figure 35. The dots show the position in geomagnetic latitude $\left(^{\circ}\right)$ of the equatorward auroral oval boundary in dependence of the Dst index according to Starkov (1993). The crosses represent the maximum extension of the diffuse auroral luminosity toward lower geomagnetic latitudes according to Zverev et al. (2009).

line of a least squares fit:

$\Phi^{\prime}=77.3^{\circ}-9.8^{\circ} \log |D s t|$

with $r=0.92$. The equatorward boundary of the visual diffuse luminosity is shifted by $\sim 7^{\circ}$ toward lower latitudes relative to the equatorward boundary of the auroral oval.

Large-scale undulations on the equatorward boundary of diffuse aurora on the evening side are observed at times during magnetic storm periods. These undulations were first discovered by Lui et al. (1982) in DMSP spacecraft photographs and then examined in several reports (e.g. Nishitani et al., 1994; Zhang et al., 2005; Henderson et al., 2010). Undulations were observed for about $0.5-3.5 \mathrm{~h}$ with amplitudes ranging from several tens to several hundreds of kilometres and wavelengths from about 200 to $900 \mathrm{~km}$. In their study, Lui et al. (1982) proposed that the Kelvin-Helmholtz instability could be considered as a possible mechanism for the observed fluctuations. However, using an instability analysis that was carried out by Viñas and Madden (1986), Kelley (1986) showed that the normal shear at the plasmapause appears to be too small for an instability to occur. He suggested instead that undulations could be driven by the much larger velocity shear associated with polarisation jets (SAPS, Foster and Burke, 2002). SAPS are formed due to the penetration of hot ring current protons further equatorward than electrons during magnetic storms or intense substorms.

Strong intensifications of $630.0 \mathrm{~nm}$ emissions were observed at mid- and low-latitudes equatorward of the diffuse aurora zone during intervals of intense disturbances including magnetic storms. They were called $\mathrm{M}$-arcs (Roach and Roach, 1963) or stable auroral red (SAR) arcs (Rees and
Roble, 1975; Baumgardner et al., 2007). SAR arcs are one of the auroral luminosity types, because their appearance is usually connected with an intensification of the geomagnetic activity and because their positioning is related to the geomagnetic field. The intensity of SAR arcs reached several kilo-Rayleighs during the IGY period (Marovich and Roach, 1963), but during intense geomagnetic storms it attained $\sim 13 \mathrm{kR}$ (Baumgardner et al., 2007) or even a few tens of kilo-Rayleighs (Barbier, 1960).

Marovich and Roach (1963) investigated the position of SAR arcs with observations at the station Rapid City $(\Phi=$ $53.1^{\circ}$ ) during the IGY. These arcs were identified in 23 cases. The authors discovered a significantly increased probability for the arc's appearance at $\Phi \sim 53^{\circ}$. Based on such a position of the SAR arc, Marovich and Roach (1963) evaluated the gap between the auroral oval and the SAR arc as of $\sim 14^{\circ}$ width (i.e. between $\Phi \sim 67^{\circ}$ and $\Phi \sim 53^{\circ}$ ). Allowing for an equatorward shift of the auroral oval during magnetic storms to $\sim 60^{\circ}$ (see Fig. 35), the whole latitudinal interval between the oval and the SAR arc near $\Phi \sim 53^{\circ}$ is then filled with diffuse auroral luminosity (Zverev et al., 2009).

Unusually bright SAR arcs can appear during very strong geomagnetic storms ("superstorms") due to the heat flow from the ring current ions via heated electrons to the ionospheric atomic oxygen plasma just equatorward of the plasmapause location. During these rare cases, there appears a latitudinal gap between the diffuse auroral boundary (DAB) and the SAR arc (Baumgardner et al., 2007).

Usually the ring current was proposed as the main energy source for SAR arcs; it allows the long-term persistence of the arcs and it is also indicated as the source due to the high correlation between the SAR appearance and the Dst index level (Rees and Akasofu, 1963). As a result of the scientific findings during the IGY, the formation of a SAR arc occurs close to the plasmapause due to the interaction of highenergy ring current ions with the cold plasmaspheric plasma. The SAR arcs at $630.0 \mathrm{~nm}$ can be driven by field-aligned magnetospheric energy transport from the ring current into the ionosphere near the plasmapause location. Various mechanisms of energy transfer from the ring current ions to the electrons of the upper atmosphere have been proposed: due to Coulomb collisions (Cole, 1965), heating of the electrons because of ion-cyclotron waves (Cornwall et al., 1971; Lundblad and Søraas, 1978), and owing to the presence of kinetic Alfvén waves (Lanzerotti et al., 1978). Waves, which are generated in the magnetosphere, heat the cold plasmaspheric electrons. These electrons flow along the magnetic field lines into the ionosphere and cause a luminescence in the upper atmosphere (SAR arcs) with red emissions at $\sim 400 \mathrm{~km}$ altitude. Therefore, the character of the energy source in SAR arcs differs from the sources of auroral luminosity at higher latitudes. Zverev et al. (2012) argue that the plasma, which participates in the magnetospheric convection, can be an energy source for the SAR arcs. The diffuse luminosity equatorward of the auroral oval and the luminosity in the SAR 
arcs are caused by electron precipitations of one and the same source.

The frequency of SAR arc occurrence and its relation to the diffuse auroral luminosity was investigated for two solar cycles from 1988 to 2006 at the station Maimaga $\left(\Phi^{\prime}=\right.$ $57.0^{\circ}$ ) by Alekseev and Ievenko (2000) and Alekseev and Ievenko (2008). Detailed information about the morphology and dynamics of the midlatitude auroral luminosity was presented by Ievenko (1993), Ievenko (1999), Alekseev and Ievenko (2000) and Ievenko and Alexeev (2004). Out of 620 nights of observations, there were 225 records of nighttime SAR arcs. The appearance of SAR arcs at midlatitudes seems therefore not to be that exotic and related to the development of geomagnetic storms, but it is a rather typical phenomenon. The most likely magnetic latitude of their occurrence is, according to the results of these investigations, $\Phi^{\prime} \sim 55^{\circ}$ with an average intensity of $\sim 0.15 \mathrm{kR}$.

Figure 36 shows a typical case for the observations of both diffuse luminosity and SAR arcs from the evening to the morning hours on 4 December 1989 (Ievenko and Alexeev, 2004). The SAR arc, the boundaries of which were determined relative to the background luminosity of the nighttime sky, moved slowly equatorward in the course of the night from evening to morning hours MLT (Fig. 36a). Its latitudinal position was determined by assuming $450 \mathrm{~km}$ as the height of luminosity. The low-latitude portion of the diffuse aurora with a green emission northward of the SAR arc (at $110 \mathrm{~km}$ altitude) is signified with the $0.5 \mathrm{kR}$ isoline. The SAR arc position and intensity $(\sim 0.18 \mathrm{kR})$ was quite stable during the relatively quiet geomagnetic conditions until $\sim 17: 40 \mathrm{UT}$ (Fig. 36b).

The beginning of the disturbance is accompanied by an increasing velocity of the arc's motion toward the Equator and an intensity increase up to $\sim 0.5 \mathrm{kR}$. A gap of $\sim 1-2^{\circ}$ in latitude between the diffuse aurora boundary and the SAR arc was recorded during the quiet period. With increasing geomagnetic activity and with a shift of the latitudinal crosssection from dusk to dawn, the gap width between the DAB and the SAR arc increases to 3-4 ${ }^{\circ}$. It is possible to assume that the gap of $1-4^{\circ}$ in Fig. 36 is not connected with an absence of auroral luminosity inside the gap, but rather with the following conditions:

1. Usage of 557.7 and $630.0 \mathrm{~nm}$ emissions for diffuse luminosity and SAR arc, respectively.

2. The isoline of $0.5 \mathrm{kR}$ is considered as the equatorward boundary of the diffuse luminosity.

Less intense diffuse luminosity should also be taken into account for the determination of the DAB position.

These observations were complemented by measurements of precipitating electrons on board of the DMSP satellite F9 as provided in the paper of Zverev et al. (2012). DMSP satellites move in a near circular sun-synchronous orbit at $\sim 840 \mathrm{~km}$ altitude. The spacecraft devices allowed
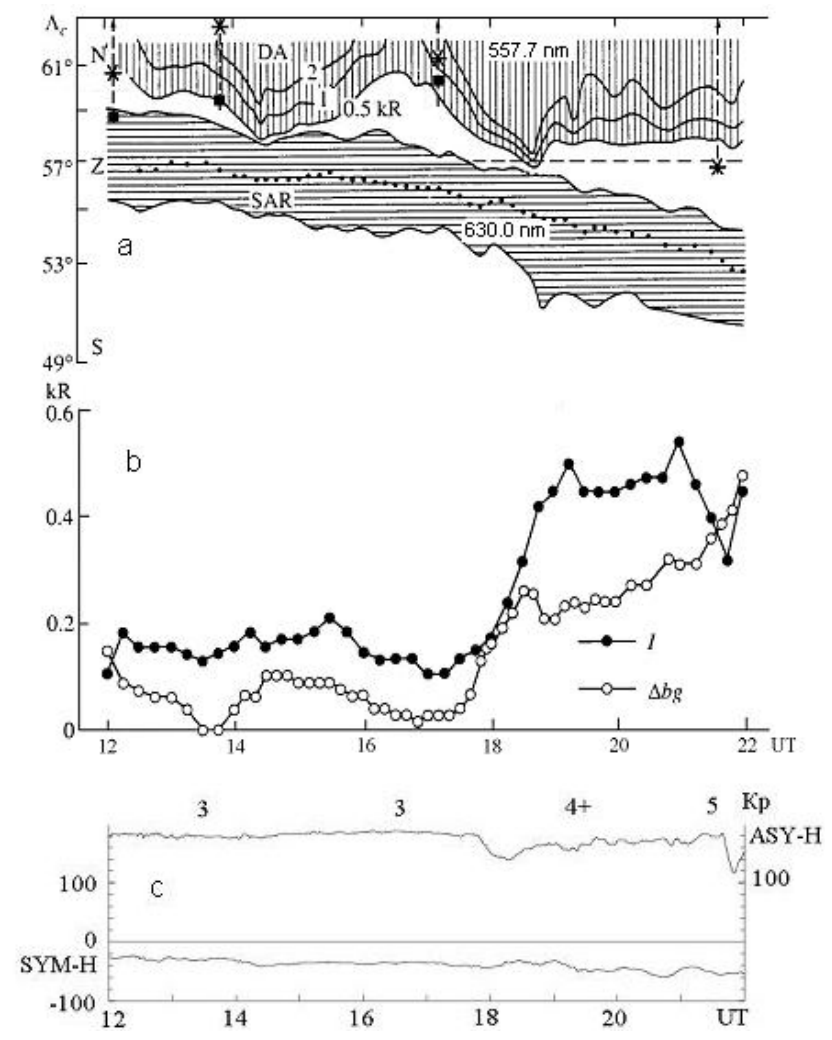

Figure 36. Modified figure from Ievenko and Alexeev (2004): (a) variation of the diffuse aurora (DA) position with $557.7 \mathrm{~nm}$ emissions and the SAR arcs (the vertical lines indicate the overflights of the DMSP satellites F9 and F8); (b) intensity variations of the SAR arcs (full dots) and the background luminosity (open circles); (c) ASY-H and SYM-H indices together with the $K p$ values written on top $(\mathrm{MLT}=\mathrm{UT}+9 \mathrm{~h})$.

measurements of the electron and ion spectra fluxes with energies between $30 \mathrm{eV}$ and $30 \mathrm{keV}$ (electron energy flux $>10^{-3} \mathrm{erg} \mathrm{cm}^{-2} \mathrm{~s}^{-1}$ ) with a $1 \mathrm{~s}$ cadence. The equatorward boundary of the electron precipitations coincided within $1^{\circ}$ magnetic latitude with the boundary of the diffuse luminosity during all satellite passes.

Figure 37a-d shows DMSP F9 energy spectra of electrons, characterising various auroras within the interval 13:46-13:50 UT. The spectrum is quasi-monochromatic at 13:46:30 UT on $\Phi^{\prime} \sim 65.5^{\circ}$ (Fig. 37a) with an electron flux density of $\sim 10^{9} \mathrm{~cm}^{-2} \mathrm{ster}^{-1} \mathrm{~s}^{-1}$ in the energy peak near $\sim 1.4 \mathrm{keV}$. Such spectra and energy fluxes are typical for discrete auroral forms at latitudes of the oval. This discrete auroral form marks the equatorward boundary of the oval. More equatorward within the diffuse auroral region, the spectrum becomes softer. At 13:46:43 UT on $\Phi^{\prime} \sim 64.8^{\circ}$ (Fig. 37b), the electron flux with an energy of $97 \mathrm{eV}$ rises by more than one order, while the flux decreases for $1.4 \mathrm{keV}$ electrons. At 13:47:38 UT on $\Phi^{\prime} \sim 62.0^{\circ}$ (Fig. 37c) the spectrum becomes even softer: electron fluxes with energies $>1 \mathrm{keV}$ are cut-off 

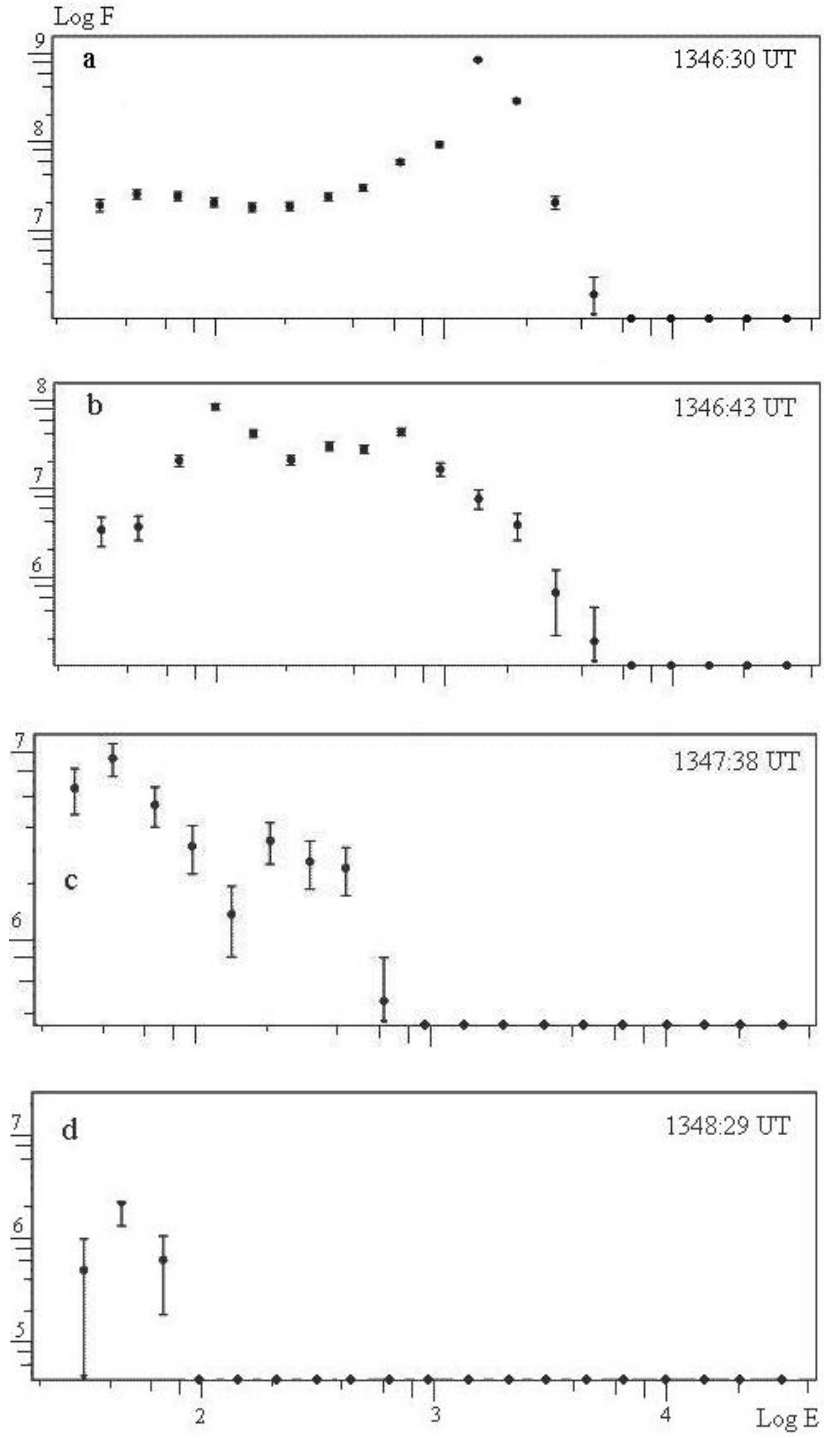

Figure 37. Electron spectra according to DMSP F9 observations on 4 December 1989 at 13:46:30 UT (a), 13:46:43 UT (b), 13:47:38 UT (c), and 13:48:29 UT (d) (Zverev et al., 2012).

and the flux with an energy of $97 \mathrm{eV}$ decreases by an order of magnitude, while the electron fluxes with energies of 31 and $45 \mathrm{eV}$ are kept at a sufficiently high level. Near the equatorward boundary of the diffuse precipitation at 13:48:29 UT on $\Phi^{\prime} \sim 59.4^{\circ}$ (Fig. 37d), only electrons of lowermost energies $(30-40 \mathrm{eV})$ remained within the electron energy spectrum with fluxes of $\sim 10^{6} \mathrm{~cm}^{-2} \mathrm{ster}^{-1} \mathrm{~s}^{-1}$. Hence, a softening of the electron spectrum toward the Equator takes place within the diffuse precipitation region, while the flux densities are kept at a quite high level. Below we will show that model estimations of the intensity of auroral luminosity at the boundary of the diffuse precipitation region approve the existence of the observed SAR arcs with an intensity of $\sim 0.2 \mathrm{kR}$ (Fig. 36b, d).
It is of interest to estimate the intensity of the $630.0 \mathrm{~nm}$ emission that is excited by the observed flux of low-energy electrons near the plasmapause. This is useful in order to explain, whether such an electron flux can constitute the source for SAR arcs at the border of the diffuse luminosity region.

The most effective excitation of red diffuse auroras is due to electrons with energies of $0.01-1.0 \mathrm{keV}$, while for SAR arcs it is $<0.01 \mathrm{keV}$. Such electrons deposit at altitudes of $200-500 \mathrm{~km}$, where they excite metastable emissions of $\mathrm{O}\left({ }^{1} D\right)$ and $\mathrm{O}\left({ }^{1} S\right)$ atomic oxygen, corresponding to red and green luminescence, respectively. At such altitudes the deactivation of excited metastable states occurs due to collisions. The excitation level of atomic oxygen emissions is low $(\sim 5 \mathrm{eV})$ and the collision cross section for the excitation processes close to the threshold value is large. All this is in favour of the red line emission of atomic oxygen in case of precipitating low-energy electrons. The intensity of such electron fluxes above SAR arcs was measured by Slater et al. (1987) on board of the Dynamics Explorer 2 satellite. The results of coordinated measurements of both low-energy electron fluxes by satellites and the intensity of $630.0 \mathrm{~nm}$ emissions by ground-based photometers have revealed the close correlation between these two phenomena. It has been shown that electrons with energies $E<0.01 \mathrm{keV}$ are the main source of energy, the precipitation of which is essential to explain the observed amount of atmospheric heating and the intensity of SAR arc emissions. The latter amounts to $\sim 2 \mathrm{kR}$ according to satellite measurements, while the electron fluxes with energies of 5.1 and $8.8 \mathrm{eV}$ were $\sim 10^{8} \mathrm{~cm}^{-2} \mathrm{~s}^{-1} \mathrm{eV}^{-1}$. The density of electron fluxes with energies of $0.03-0.04 \mathrm{keV}$ were two orders of magnitude smaller with values of $\sim 8 \cdot 10^{5}$ or $\sim 3 \cdot 10^{7} \mathrm{~cm}^{-2} \mathrm{~s}^{-1} \mathrm{eV}^{-1}$. This is one order of magnitude larger than the electron flux densities of the same energy, which were recorded with the DMSP satellite on 4 December 1989, 13:48:29 UT, above the SAR arc. Such low-energy electron fluxes can therefore excite a diffuse red luminosity with an intensity of $\sim 0.2 \mathrm{kR}$ near the plasmapause. The appearance of luminosity of this intensity at midlatitudes would be interpreted as a SAR arc. Its existence can be considered as evidence for the interrelation of plasma fluxes with auroral energy, which are transferred by magnetospheric convection from the central plasma sheet to the plasmapause, producing a SAR arc.

At the nightside magnetosphere between $\sim 6$ and $\sim 3 R_{\mathrm{E}}$ (i.e. between the inner boundary of the plasma sheet in the magnetospheric tail and the plasmapause, the outer boundary of the plasmaspheric thermal plasma close to Earth) there are two different populations of ionised particles: the soft plasma particles coming from the plasma sheet with energies of less than $1 \mathrm{keV}$ and the energetic electrons with $\epsilon>30 \mathrm{keV}$ which are trapped in the geomagnetic field (outer radiation belt). The diffusive emission in the atmosphere at latitudes, where this region maps along the geomagnetic field lines, is caused by the low-energy population. 
Zverev et al. (2012) concluded from model calculations of Rassoul et al. (1993) that the auroral luminosity in the form of SAR arcs of low intensity can be related to the precipitation of low-energy electrons from the magnetosphere in the neighbourhood of the plasmapause. The variations of the parameters of corpuscular particles in the inner magnetosphere and in the midlatitude ionosphere are similar: with decreasing latitude one observes a softening of the spectra, a decrease of the energy density of the precipitating flux, and an attenuation of the auroral luminosity. It seems therefore to be obvious to relate the appearance of SAR arcs near the equatorward border of the diffuse precipitation (DPB) with electron fluxes of low energy, having in mind the large efficiency of excitation processes for red emissions with electrons $<10 \mathrm{eV}$.

Thus the existence of SAR arcs with intensities $<1.0 \mathrm{kR}$ for the $630.0 \mathrm{~nm}$ emission can be related to precipitating low-energy electrons of the plasma sheet. The arcs are located near the equatorward boundary of the diffuse electron precipitation region, where their energy decreases to values $<0.01 \mathrm{keV}$. Such a position of weak SAR arcs is caused both by the character of energy change of auroral particles due to the large-scale plasma convection in the nightside magnetosphere and by the character of excitation and deactivation processes of $630.0 \mathrm{~nm}$ emissions in the upper atmosphere.

\section{Conclusions}

This review presents research results on spectral and morphological characteristics of discrete auroral forms in a historical retrospective. These investigations led soon after the IGY period (1957-1958) to the emergence of conceptions about the auroral oval and auroral substorms. The concept of the auroral oval was developed by Russian scientists and became broadly supported from leading specialists in other countries. The concept of an auroral substorm was proposed in the United States and later specified and supplemented by Russian researchers. These two conceptions represent basic paradigms of the present-day solar-terrestrial physics. They are nowadays broadly applied to describe the entirety of geophysical phenomena at high latitudes.

An immense amount of observational material was accumulated by the international scientific community during the IGY/IGC and IQSY, which comprised ground-based optical and magnetometer observations. These data and further ground-based observations later on, which were supplemented by spacecraft observations in various regions of Earth's environment, allowed refining and extending the conceptions of the auroral oval and the auroral substorm considerably.

Aurorae were divided into dayside and nightside parts. Having different spectral and morphological characteristics, the dayside and nightside aurorae constitute a single annular zone around the geomagnetic pole (auroral oval). The day- time luminosity, in particular the region near midday, which maps along the geomagnetic field lines to the dayside magnetopause, is closely linked with interaction processes between the magnetosphere and the magnetic field and plasma of the solar wind. However, the nighttime aurorae map into the magnetospheric tail, which determines their particular characteristics and relates them closely to the processes of auroral substorm developments. The magnetospheric substorm, one aspect of which are auroral substorms, represents an intricate physical process. It is determined by the energy transfer from the solar wind into the magnetosphere as well as by processes, which take place within the magnetosphere itself, in its plasma sheet and in the ring current. In this way the auroral substorm is connecting in itself the structural unity and disparity of the dayside and nightside parts of the auroral oval.

The complexity and intricacy of the inner magnetospheric processes matches with the auroral substorm development. The existence of microprocesses emerges within substorms, which are accompanied by sudden broadenings of bright nighttime auroras in latitudinal and longitudinal directions. Long-lasting periods with strong magnetic activity can occur, but they do not show any indications of substorm developments. Such events were termed steady magnetospheric convection (SMC) intervals, which are observed during prolonged periods of southward directed IMF. A scheme of the global distribution of discrete and diffuse auroras during the SMC period was drawn.

Polar cap auroras (PCAs) were identified and analysed in detail as a particular type of auroras poleward of the auroral oval. Discrete forms of PCA are extended in the sunward direction and are recorded mainly during periods of northward IMF. Some scientists argue that PCAs are situated at closed geomagnetic field lines. Such a hypothesis presumes a complicated spatial distribution and structure of the magnetospheric plasma sheet during magnetic silence.

The auroral structure and its dynamics during disturbance periods appears to be closely connected with the magnetospheric plasma structure. This opens new ways for the diagnostics of the near-Earth environment by means of groundbased and satellite observations of the auroras and provides likewise a strong stimulus for the further development of basic research in the ionosphere and magnetosphere.

Acknowledgements. Work on this review at the Polar Geophysical Institute (V.G. V. and V.L. Z.) was supported by the RFBR Grant 09-12-00273 and Program no. 4 of the Russian Academy of Science. Work at GFZ German Research Centre for Geosciences Potsdam (M. F.) was supported by Deutsche Forschungsgemeinschaft (DFG). We thank S. A. Chernouss and V. Yu. Zhiganov for providing the auroral photographs.

Edited by: T. Kikuchi

Reviewed by: P. E. Sandholt and two anonymous referees 
Appendix A

Table A1. Coordinates of stations for the observation of auroral luminosity; the last column indicates Corrected Geomagnetic Latitudes.

\begin{tabular}{|c|c|c|c|c|c|c|}
\hline \multirow[t]{2}{*}{ No. } & \multirow[t]{2}{*}{ Station } & \multicolumn{2}{|c|}{ Geomagnetic } & \multicolumn{2}{|c|}{ Geographical } & \multirow{2}{*}{$\begin{array}{r}\text { Corr. Geom. } \\
\text { Latitude }\end{array}$} \\
\hline & & Latitude & Longitude & Latitude & Longitude & \\
\hline 1 & Thule & $88.0^{\circ} \mathrm{N}$ & $1.2^{\circ} \mathrm{E}$ & $76.6^{\circ} \mathrm{N}$ & $68.9^{\circ} \mathrm{W}$ & $86.1^{\circ} \mathrm{N}$ \\
\hline 2 & Alert & $85.9^{\circ} \mathrm{N}$ & $165.7^{\circ} \mathrm{E}$ & $82.5^{\circ} \mathrm{N}$ & $62.3^{\circ} \mathrm{W}$ & $86.7^{\circ} \mathrm{N}$ \\
\hline 3 & Nord & $80.8^{\circ} \mathrm{N}$ & $133.3^{\circ} \mathrm{E}$ & $81.6^{\circ} \mathrm{N}$ & $16.6^{\circ} \mathrm{W}$ & $80.7^{\circ} \mathrm{N}$ \\
\hline 4 & Godhavn & $79.9^{\circ} \mathrm{N}$ & $32.5^{\circ} \mathrm{E}$ & $69.3^{\circ} \mathrm{N}$ & $53.5^{\circ} \mathrm{W}$ & $77.6^{\circ} \mathrm{N}$ \\
\hline 5 & Arctic Ice Flow & $78.3^{\circ} \mathrm{N}$ & $156.0^{\circ} \mathrm{W}$ & $84.5^{\circ} \mathrm{N}$ & $148.0^{\circ} \mathrm{W}$ & $79.4^{\circ} \mathrm{N}$ \\
\hline 6 & Arctica 2 & $77.5^{\circ} \mathrm{N}$ & $161.0^{\circ} \mathrm{W}$ & $86.0^{\circ} \mathrm{N}$ & $186.0^{\circ} \mathrm{W}$ & $78.5^{\circ} \mathrm{N}$ \\
\hline 7 & Barentsburg & $74.4^{\circ} \mathrm{N}$ & $129.9^{\circ} \mathrm{E}$ & $78.1^{\circ} \mathrm{N}$ & $14.2^{\circ} \mathrm{E}$ & $75.2^{\circ} \mathrm{N}$ \\
\hline 8 & Godthåb & $74.8^{\circ} \mathrm{N}$ & $29.6^{\circ} \mathrm{E}$ & $64.2^{\circ} \mathrm{N}$ & $308.3^{\circ} \mathrm{E}$ & $73.3^{\circ} \mathrm{N}$ \\
\hline 9 & Cap Thordsen & $74.5^{\circ} \mathrm{N}$ & $131.7^{\circ} \mathrm{E}$ & $78.5^{\circ} \mathrm{N}$ & $15.7^{\circ} \mathrm{E}$ & $74.3^{\circ} \mathrm{N}$ \\
\hline 10 & Pyramida & $74.5^{\circ} \mathrm{N}$ & $130.8^{\circ} \mathrm{E}$ & $78.2^{\circ} \mathrm{N}$ & $15.2^{\circ} \mathrm{E}$ & $74.8^{\circ} \mathrm{N}$ \\
\hline 11 & Tikhaya Bay & $71.5^{\circ} \mathrm{N}$ & $153.3^{\circ} \mathrm{E}$ & $80.3^{\circ} \mathrm{N}$ & $52.8^{\circ} \mathrm{E}$ & $74.4^{\circ} \mathrm{N}$ \\
\hline 12 & Heiss Island & $71.5^{\circ} \mathrm{N}$ & $155.3^{\circ} \mathrm{E}$ & $80.7^{\circ} \mathrm{N}$ & $56.2^{\circ} \mathrm{E}$ & $74.6^{\circ} \mathrm{N}$ \\
\hline 13 & Wiese Island & $69.3^{\circ} \mathrm{N}$ & $163.8^{\circ} \mathrm{E}$ & $79.9^{\circ} \mathrm{N}$ & $77.0^{\circ} \mathrm{E}$ & $73.4^{\circ} \mathrm{N}$ \\
\hline 14 & Point Barrow & $68.5^{\circ} \mathrm{N}$ & $118.8^{\circ} \mathrm{W}$ & $71.6^{\circ} \mathrm{N}$ & $156.4^{\circ} \mathrm{W}$ & $69.9^{\circ} \mathrm{N}$ \\
\hline 15 & Fort Yukon & $66.7^{\circ} \mathrm{N}$ & $102.9^{\circ} \mathrm{W}$ & $66.6^{\circ} \mathrm{N}$ & $145.3^{\circ} \mathrm{W}$ & $67.0^{\circ} \mathrm{N}$ \\
\hline 16 & Cape Chelyskin & $66.3^{\circ} \mathrm{N}$ & $176.5^{\circ} \mathrm{E}$ & $77.8^{\circ} \mathrm{N}$ & $104.3^{\circ} \mathrm{E}$ & $71.2^{\circ} \mathrm{N}$ \\
\hline 17 & Kiruna & $65.4^{\circ} \mathrm{N}$ & $115.8^{\circ} \mathrm{E}$ & $67.9^{\circ} \mathrm{N}$ & $20.5^{\circ} \mathrm{E}$ & $64.5^{\circ} \mathrm{N}$ \\
\hline 18 & Wrangel Island & $64.7^{\circ} \mathrm{N}$ & $133.8^{\circ} \mathrm{W}$ & $71.0^{\circ} \mathrm{N}$ & $178.6^{\circ} \mathrm{W}$ & $66.6^{\circ} \mathrm{N}$ \\
\hline 19 & College & $64.7^{\circ} \mathrm{N}$ & $103.4^{\circ} \mathrm{W}$ & $64.9^{\circ} \mathrm{N}$ & $147.9^{\circ} \mathrm{W}$ & $64.9^{\circ} \mathrm{N}$ \\
\hline 20 & Murmansk & $64.1^{\circ} \mathrm{N}$ & $126.5^{\circ} \mathrm{E}$ & $69.0^{\circ} \mathrm{N}$ & $33.1^{\circ} \mathrm{E}$ & $64.9^{\circ} \mathrm{N}$ \\
\hline 21 & Loparskaya & $63.8^{\circ} \mathrm{N}$ & $126.6^{\circ} \mathrm{E}$ & $68.6^{\circ} \mathrm{N}$ & $33.3^{\circ} \mathrm{E}$ & $64.8^{\circ} \mathrm{N}$ \\
\hline 22 & Dixon Island & $63.0^{\circ} \mathrm{N}$ & $161.5^{\circ} \mathrm{E}$ & $73.5^{\circ} \mathrm{N}$ & $80.4^{\circ} \mathrm{E}$ & $67.9^{\circ} \mathrm{N}$ \\
\hline 23 & Tixie Bay & $60.4^{\circ} \mathrm{N}$ & $168.7^{\circ} \mathrm{W}$ & $71.6^{\circ} \mathrm{N}$ & $128.9^{\circ} \mathrm{E}$ & $65.2^{\circ} \mathrm{N}$ \\
\hline 24 & Kazachye & $60.0^{\circ} \mathrm{N}$ & $163.7^{\circ} \mathrm{W}$ & $70.8^{\circ} \mathrm{N}$ & $136.3^{\circ} \mathrm{E}$ & $64.4^{\circ} \mathrm{N}$ \\
\hline 25 & Maimaga & $56.5^{\circ} \mathrm{N}$ & $200.0^{\circ} \mathrm{E}$ & $67.0^{\circ} \mathrm{N}$ & $129.0^{\circ} \mathrm{E}$ & $57.0^{\circ} \mathrm{N}$ \\
\hline 26 & Rapid City & $53.1^{\circ} \mathrm{N}$ & $42.6^{\circ} \mathrm{W}$ & $44.0^{\circ} \mathrm{N}$ & $103.1^{\circ} \mathrm{W}$ & $53.9^{\circ} \mathrm{N}$ \\
\hline 27 & Yakutsk & $51.0^{\circ} \mathrm{N}$ & $166.2^{\circ} \mathrm{W}$ & $62.1^{\circ} \mathrm{N}$ & $129.7^{\circ} \mathrm{E}$ & $55.8^{\circ} \mathrm{N}$ \\
\hline 28 & Dumont d'Urville & $75.0^{\circ} \mathrm{S}$ & $130.0^{\circ} \mathrm{W}$ & $66.7^{\circ} \mathrm{S}$ & $140.1^{\circ} \mathrm{E}$ & $80.4^{\circ} \mathrm{S}$ \\
\hline 29 & Wilkes & $77.7^{\circ} \mathrm{S}$ & $179.6^{\circ} \mathrm{E}$ & $66.3^{\circ} \mathrm{S}$ & $111.5^{\circ} \mathrm{E}$ & $80.7^{\circ} \mathrm{S}$ \\
\hline 30 & Scott Base & $79.0^{\circ} \mathrm{S}$ & $65.6^{\circ} \mathrm{W}$ & $77.9^{\circ} \mathrm{S}$ & $166.8^{\circ} \mathrm{E}$ & $80.1^{\circ} \mathrm{S}$ \\
\hline 31 & Vostok & $90.0^{\circ} \mathrm{S}$ & $171.8^{\circ} \mathrm{E}$ & $78.5^{\circ} \mathrm{S}$ & $106.9^{\circ} \mathrm{E}$ & $83.5^{\circ} \mathrm{S}$ \\
\hline
\end{tabular}




\section{References}

Ackerson, K. L. and L. A. Frank, Correlated satellite measurements of low-energy electron precipitation and ground-based observations of a visibly auroral arc, J. Geophys. Res., 77, 1128-1136, 1972.

Akasofu, S.-I., The dynamical morphology of the aurora polaris, J. Geophys. Res., 68, 1667-1673, 1963.

Akasofu, S.-I.: The development of the auroral substorm, Planet. Space Sci., 12, 273-282, 1964a.

Akasofu, S.-I.: The latitudinal shift of the auroral belt, J. Atmos. Terr. Phys., 26, 1167-1174, 1964b.

Akasofu, S.-I.: Dynamic morphology of auroras, Space Sci. Rev., 4, 498-540, 1965.

Akasofu, S.-I.: Polar and magnetospheric substorms, D. Reidel Pub. Co., Dordrecht, The Netherlands, 316 pp., 1968.

Akasofu, S.-I.: Midday auroras and polar cap auroras, Geofysiske Publikasjoner, 29, 73-86, 1972a.

Akasofu, S.-I.: Midday auroras and magnetospheric substorm, J. Geophys. Res., 77, 244-247, 1972b.

Akasofu, S.-I.: Midday auroras at the South Pole during magnetospheric substorm, J. Geophys. Res., 7, 2303-2308, 1972c.

Akasofu, S.-I.: Photographs of the front of the expanding auroral bulge during an auroral substorm, Planet. Space Sci., 20, 821822, 1972d.

Akasofu, S.-I.: A study of auroral displays photographed from the DMSP-2 satellite and from the Alaska meridian chain of stations, Space Sci. Rev., 16, 617-725, 1974a.

Akasofu, S.-I.: Discrete, continuous, and diffuse auroras, Planet. Space Sci., 22, 1723-1726, 1974b.

Akasofu, S.-I.: Recent progress in studies of DMSP auroral photographs, Space Sci. Rev., 19, 169-215, 1976.

Akasofu, S.-I.: Several 'controversial' issues on substorms, Space Sci. Rev., 113, 1-40, 2004.

Akasofu, S.-I. and Chapman, S.: A large change in the distribution of the auroras during the 11 February 1958 magnetic storm, J. Atmos. Terr. Phys., 24, 740-742, 1962.

Akasofu, S.-I., Chapman, S., and Meng, C.-I.: The polar electrojet, J. Atmos. Terr. Phys., 27, 1275-1305, 1965.

Akasofu, S.-I., Wilson, C. R., Snyder, L., and Perreault, P.: Results from a meridian chain of observatories in the Alaskan sector, Planet. Space Sci., 19, 477-482, 1971.

Akasofu, S.-I., Perreault, P. D., Yasuhara, F., and Meng, C.-I.: Auroral substorms and the interplanetary magnetic field, J. Geophys. Res., 78, 7490-7508, 1973.

Akasofu, S.-I., Lui, A. T. Y., and Meng, C.-I.: The importance of auroral features in the search for substorm onset, J. Geophys. Res., 115, A08218, doi:10.1029/2009JA014960, 2010.

Alekseev, V. N. and Ievenko, I. B.: Occurrence frequency of SAR arcs during solar-activity cycle 22 , Geomagn. Aeron., 40, 511513, 2000 (in Russian).

Alekseev, V. N. and Ievenko, I. B.: SAR arc occurrence frequency during cycle 23 of solar activity, Geomagn. Aeron., 48, 446-449, 2008 (in Russian).

Alekseev, V. N., Velichko, N. A., and Nadubovich, Y. A.: Study of the heights of luminosity and the location of the boundary of 6300 A background luminosity, in: Transactions on "Physics of the Upper Atmosphere”, 3, 124-133, Yakutsk, USSR, 1975.
Alekseev, V. N., Zickratch, E. K., Mamrukov, A. P., Filippov, L. D., Khalipov, V. L., and Shalbaeva, I. V.: Covariations of equatorial boundaries of $\mathrm{f} 2 \mathrm{~s}$ oblique ionospheric reflections and of diffuse emissions of $630.0 \mathrm{~nm}$, in Magnetospheric Substorm and Geophysical Events, 23-31, Yakutsk, USSR, 1980.

Alfvén, H.: Cosmical electrodynamics, International Series of Monographs on Physics, Clarendon Press, Oxford, 1950.

Alfvén, H.: On the electric field theory of magnetic storms and aurorae, Tellus, 7, 50-64, 1955.

Alfvén, H.: On the theory of magnetic storms and auroras, Tellus, 10, 104-116, 1958.

Allen, J. H., Abston, C. C., and Morris, L. D.: Auroral electrojet magnetic activity indices AE(11) for 1973, Tech. Rep. Report UAG-47, NOAA, Boulder, Colorado, 1975.

Anger, C. D., Lui, A. T. Y., and Akasofu, S.-I.: Observations of the auroral oval and westward travelling surge from ISIS 2 satellite and Alaska meridian all-sky cameras, J. Geophys. Res., 78, 3020-3026, 1973.

Ångström, A. J.: Spectrum des Nordlichts, Annales of Physics, 173, 161-163, 1869.

Annals of the IGY, Ascaplots: half-hourly all-sky camera plots from 114 stations for the period 1957-1958, Vol. 20, parts 1 and 2, International Council of Scientific Unions. Special Committee for the IGY Ed., Pergamon Press, Oxford, 1962.

Annals of the IGY, Auroral Visoplots, vol. 29, edited by: McInnes, B., Pergamon Press, Oxford, 1964.

Barbier, D.: L'arc auroral stable, Ann. Geophys., 16, 544-549, 1960 , http://www.ann-geophys.net/16/544/1960/.

Baumgardner, J., Wroten, J., Semeter, J., Kozyra, J., Buonsanto, M., Erickson, P., and Mendillo, M.: A very bright SAR arc: implications for extreme magnetosphere-ionosphere coupling, Ann. Geophys., 25, 2593-2608, doi:10.5194/angeo-25-25932007, 2007.

Berkey, F. T., Cogger, L. L., Ismail, S., and Kamide, Y.: Evidence for a correlation between sun-aligned arcs and the interplanetary magnetic field direction, Geophys. Res. Lett., 3, 145-147, 1976.

Birkeland, K.: The Norwegian Aurora Polaris Expedition of 19021903: On the cause of magnetic storms and the origin of terrestrial magnetism, vol. 1, Christiana, H. Aschehoug; Leipzig, Johann Ambrosius Barth; London, New York, Longmans, Green \& Co.; Paris, C. Klincksieck, First Section, 1908.

Birkeland, K.: The Norwegian Aurora Polaris Expedition of 19021903: On the cause of magnetic storms and the origin of terrestrial magnetism, vol. 2, Christiana, H. Aschehoug; Leipzig, Johann Ambrosius Barth; London, New York, Longmans, Green \& Co.; Paris, C. Klincksieck, Christiania, Second Section, 1913.

Bostrøm, R.: A model of the auroral electrojets, J. Geophys. Res., 69, 4983-4994, 1964.

Buchau, J., Whalen, J. A., and Akasofu, S.-I.: On the continuity of the auroral oval, J. Geophys. Res., 75, 7147-7160, 1970.

Burdo, O. A.: Relation of regular and irregular variations of the geomagnetic field, high latitudes, in: Transactions of the Arctic and Antarctic Institute, Vol. 223, 21-45, AARI, Leningrad, USSR, 1960.

Carlheim-Gyllenskiöld, G.: Exploration internationale des regions polaires, 1882-1883, Observations faites au Cap Thordsen, Spitzbergen, par l'expedition suedoise, vol. II.1: Aurores bore- 
ales, L'Académie Royale des Sciences du Suède, Stockholm, Sweden, 30 plates, 409 pp., 1887.

Chapman, S.: The electric current systems of magnetic storms, Terr. Magn. Atmos. Elect., 40, 349-370, 1935.

Chapman, S.: The aurora in middle and low latitudes, in: Annals of the International Geophysical Year (I.G.Y.), Vol. 4, edited by: I. C. of Scientific Unions. Special Committee for the IGY, 25-40, Perganon Press, Oxford, 1957.

Chapman, S.: Earth storms: retrospect and prospect, J. Phys. Soc. Jpn., 17, 6-16, 1962.

Chernouss, S. A.: Ideas of Lomonosov in auroral research, Geophysica, 48, 105-117, 2012.

Chua, D., Brittnacher, M., Parks, G., Germany, G., and Spann, J.: A new auroral feature: The nightside gap, Geophys. Res. Lett., 25, 3747-3750, 1998.

Cogger, L. L., Murphree, J. S., and Anger, C. D.: High space and time resolution ultraviolett auroral images from the Viking spacecraft, Phys. Scripta, 37, 432-436, 1988.

Cole, K. D.: Stable auroral red arcs, sinks for energy of Dst main phase, J. Geophys. Res., 70, 1689-1706, 1965.

Cornwall, J. M., Coroniti, F. V., and Thorne, R. M.: Unified theory of SAR arc formation at the plasmapause, J. Geophys. Res., 76, 4428-4445, 1971.

Craven, J. D., Murphree, J. S., Frank, L. A., and Cogger, L. L.: Simultaneous optical observations of transpolar arcs in the two polar caps with De-1 and Viking, Geophys. Res. Lett., 18, $2297-$ 2301, 1991.

Cumnock, J. A., Sharber, J. R., Heelis, R. A., Hairston, M. R., and Craven, J. D.: Evolution of the global aurora during positive IMF $\mathrm{Bz}$ and varying IMF $B_{y}$ conditions, J. Geophys. Res., 102, 17489-17497, 1997.

Dandakar, B. S. and C. P. Pike: The midday discrete auroral gap, J. Geophys. Res., 83, 4227-4236, 1978.

Danielsen, C.: Auroral observations at Thule 1961-1965, in: Geophys. Papers, R-9, Danish Meteorol. Inst., Charlottenlund, Denmark, 1969.

Davis, T. N.: The morphology of polar aurora, J. Geophys. Res., 65, 3497-3500, 1960.

Davis, T. N.: The morphology of the auroral displays of 1957-1958. 2. Detailed analyses of Alaska data and analyses of high-latitude data, J. Geophys. Res., 67, 75-110, 1962.

Davis, T. N.: Negative correlation between polar-cap visual aurora and magnetic activity, J. Geophys. Res., 68, 4447-4453, 1963.

De La Beaujardiere, O., Lyons, L. R., Ruohoniemi, J. M., FriisChristensen, E., Danielsen, C., Rich, F. J., and Newell, P. T.: Quiet-time intensifications along the poleward auroral boundary near midnight, J. Geophys. Res., 99, 287-298, doi:10.1029/93JA01947, 1994.

Denholm, J. V. and Bond, F. R.: Orientation of polar auroras, Aust. J. Phys., 14, 193-198, 1961.

Despirak, I., Lubchich, A., Yahnin, A. G., Galperin, Y. I., Vennerstrom, S., Aulamo, O., and Craven, J. D.: Region of cusp-like precipitation in dayside high latitudes during steady magnetospheric convection, Geomagn. Aeron., 34, 5-10, 1994 (in Russian).

Eather, R. H.: Auroral proton precipitation and hydrogen emissions, Rev. Geophys., 5, 207-285, 1967.
Eather, R. H.: Majestic lights, the aurora in science, history, and the art, American Geophysical Union, Washington, D.C., 323 pp., 1980.

Elphinstone, R. D., Jankowska, K., Murphree, J. S., and Cogger, L. L.: The configuration of the auroral distribution for interplanetary magnetic field $\mathrm{Bz}$ Northward: 1 . IMF $B_{x}$ and $B_{y}$ dependencies as observed by the Viking satellite, J. Geophys. Res., 95, 5791-5804, 1990.

Elphinstone, R. D., Murphree, J. S., Hearn, D., Cogger, L. L., Sandahl, I., Newell, P. T., Klumpar, D. M., Ohtani, S., Sauvaud, J. A., Potemra, T. A., Mursula, K., Wright, A., and Shapshak, M.: The double oval UV auroral distribution: 1. Implications for the mapping of auroral arcs, J. Geophys. Res., 100, 12075-12092, 1995.

Elvey, C. T., Leinbach, H., Hessler, J., and Noxon, J.: Preliminary studies of the distribution of aurora in Alaska, in: Transactions of the American Geophys. Union, no. 3 in 58, Washington, 1955.

Evlashin, L. S.: Space-time variations of hydrogen in auroras and their connection with magnetic disturbances, Geomagn. Aeron., 1, 54-57, 1961.

Evlashin, L. S.: On the relation between several types of auroras and absorption of radiowaves in the ionosphere, in: Geophysical Events in the Auroral Zone, 74-84, Publ. House Nauka, Leningrad, USSR, 1971.

Evlashin, L. S., Truttse, Y. I., and Feldstein, Y. I.: The effect of the ring current and polar electrojet on the oval of proton aurorae, Planet. Space Sci., 34, 859-866, 1972.

Fairfield, D.: Polar magnetic disturbances and the interplanetary magnetic field, Space Res., 8, 107-119, 1967.

Fairfield, D.: Average magnetic field configuration of the outer magnetosphere, J. Geophys. Res., 73, 7329-7338, 1968.

Feldstein, Y. I.: Geographic distribution of aurorae in the westward sector of Soviet Arctic, Problems of the Arctic, N4, 45-49, 1958.

Feldstein, Y. I.: Geographical distribution of the aurorae and azimuths of auroral arcs, in: IGY Transactions "Investigations of the aurorae", Vol. N4, 61-77, Publ. House Academy Sci. USSR, 1960.

Feldstein, Y. I.: The morphology of auroras and geomagnetism, in: IGY Transactions "Auroras and Airglow", Vol. N10, 121-125, Publ. House Academy Sci. USSR, 1963a.

Feldstein, Y. I.: On the morphology of auroras and magnetic disturbances at high latitudes, Geomagn. Aeron., 3, 227-239, 1963b (in Russian).

Feldstein, Y. I.: The auroral oval-shaped zone location on the night side of the Earth on magnetically quiet days, Geomagn. Aeron., 6, 894-900, 1966.

Feldstein, Y. I.: Auroras and associated phenomena, in SolarTerrestrial Physics, edited by: Dyer, E. R., 152-191, D. Reidel Publ. Comp., Dordrecht, 1972.

Feldstein, Y. I.: Night-time aurora and its relation to magnetosphere, Ann. Geophys., 30, 259-272, 1974, http://www.ann-geophys.net/30/259/1974/.

Feldstein, Y. I.: A quarter of a century with the auroral oval, EOS, Trans. Am. Geophys. Union, 67, 761-767, doi:10.1029/EO067i040p00761-02, 1986.

Feldstein, Y. I. and Galperin, Y. I.: The auroral luminosity structure in the high-latitude upper atmosphere, its dynamics and relationship to the large-scale structure of the Earth's magnetosphere, Rev. Geophys., 23, 217-275, 1985. 
Feldstein, Y. I. and Galperin, Y. I.: Structures of auroral precipitation in the night-time sector of the magnetosphere, Kosm. Issled., 34, 227-247, 1996 (in Russian).

Feldstein, Y. I. and Starkov, G. V.: Dynamics of auroral belt and polar geomagnetic disturbances, Planet. Space Sci., 15, 209-229, 1967a.

Feldstein, Y. I. and Starkov, G. V.: Scheme of the auroral substorm on the dayside, Geomagn. Aeron., 7, 307-309, 1967b.

Feldstein, Y. I. and Starkov, G. V.: Investigations of the auroras, in: Transactions "Investigation of aurorae", Vol. 7, 17-25, USSR Academy of Science Publishing House, Moscow, 1970a.

Feldstein, Y. I. and Starkov, G. V.: The auroral oval and the boundary of closed field lines of geomagnetic field, Planet. Space Sci., 18, 501-508, $1970 \mathrm{~b}$.

Feldstein, Y. I., Shevnina, N. F., and Lukina, L. V.: Aurora during magneto-disturbed and magneto-quiet periods, Geomagn. Aeron., 6, 312-321, 1966 (in Russian).

Feldstein, Y. I., Lukina, L. V., and Belousov, B. G.: Motion of auroras in near polar region, The newsletter. SAE, No. 69, 50-56, 1968.

Feldstein, Y. I., Isaev, S. I., and Lebedinsky, A. I.: The phenomenology and morphology of aurorae, Ann. IQSY, 4, 311-348, 1969.

Feldstein, Y. I., Starkov, G. V., and Schevina, N. F.: Motions of aurorae and electric fields in the magnetosphere, in: Morphology and physics of the polar ionosphere, 68-74, Publ. House Nauka, Leningrad, USSR, 1971 (in Russian).

Feldstein, Y. I., Newell, P. T., Sandahl, I., Woch, J., Leontyev, S. V., and Vorobjev, V. G.: Structure auroral precipitation during a theta aurora from multisatellite observations, J. Geophys. Res., 100, 17429-17442, 1995.

Feldstein, Y. I., Gromova, L. I., Woch, J., Sandahl, I., Blomberg, L., Marklund, G., and Meng, C.-I.: Structure of the auroral precipitation region in the dawn sector: relationship to convection reversal boundaries and field-aligned currents, Ann. Geophys., 19, 495-519, doi:10.5194/angeo-19-495-2001, 2001.

Feldstein, Y. I., Popov, V. A., Cumnock, J. A., Prigancova, A., Blomberg, L. G., Kozyra, J. U., Tsurutani, B. T., Gromova, L. I., and Levitin, A. E.: Auroral electrojets and boundaries of plasma domains in the magnetosphere during magnetically disturbed intervals, Ann. Geophys., 24, 2243-2276, doi:10.5194/angeo-242243-2006, 2006.

Feldstein, Y. I., Vorobjev, V. G., and Zverev, V. L.: Planetary features of aurorae: Results of the IGY (a review), Geomagn. Aeron., 50, 413-435, 2010.

Feldstein, Y. I., Vorobjev, V. G., and Zverev, V. L.: Comment on The importance of auroral features in the search for substorm onset process by Syun-Ichi Akasofu, A. T. Y. Lui, and C.-I. Meng, J. Geophys. Res., 116, A02208, doi:10.1029/2010JA016064, 2011.

Foster, J. C. and Burke, W. J.: SAPS: A new categorization for subauroral electric fields, EOS, 83, 393-394, 2002.

Foster, J. C., Fairfield, D. H., Ogilvie, K. W., and Rosenberg, T. J.: Relationship of interplanetary parameters and occurence of magnetospheric substorms, J. Geophys. Res., 76, 6971-6975, 1971.

Frank, L. A. and Ackerson, K. L.: Observations of charged particle precipitation into the auroral zone, J. Geophys. Res., 76, 36123643, 1971.

Frank, L. A., Van Allen, J. A., and Craven, J. D.: Large diurnal variation of geomagnatically trapped and precipitated electrons observed at low altitudes, J. Geophys. Res., 69, 3155-3167, 1964.
Frank, L. A., Saflekos, N. A., and Ackerson, K. L.: Electron precipitation in the postmidnight sector of the auroral zones, J. Geophys. Res., 81, 155-167, 1976.

Frank, L. A., Craven, J. D., Burch, J. L., and Winningham, J. D.: Polar views of the Earth's aurora with Dynamic Explorer, Geophys. Res. Lett., 9, 1001-1004, 1982.

Frank, L. A., Craven, J. D., and Rairden, R. I.: Images of the Earth's aurora and geocorona from the Dynamic Explorer mission, Adv. Space Res., 4, 53-68, 1985.

Frank, L. A., Craven, J. D., Gurnett, D. A., Shawhan, S. D., Weimer, D. R., Burch, J. L., Winningham, J. D., Chappell, C. R., Wait, J. H., Heelis, R. A., Maynard, N. C., Sugiura, M., Peterson, W. R., and Shelley, E. A.: The theta-aurora, J. Geophys. Res., 91, 3177-3225, 1986.

Frank, L. A., Sigwarth, J. B., Craven, J. D., Cravens, J. P., Dolan, J. S., Dvorsky, M. R., Hardebeck, P. K., Harvey, J. D., and Muller, D. W.: The visible imaging system (VIS) for the Polar spacecraft, Space Sci. Rev., 71, 297-328, 1995.

Franklin, B.: Political, Miscellaneous and Philosophical Pieces, edited by: Vaugham, B., London, Printed for J. Johnson, No. 72, St. Paul's Church-Yard, 1779.

Frey, H. U., Mende, S. B., Angelopoulos, V., and Donovan, E. F.: Substorm onset observations by IMAGE-FUV, J. Geophys. Res., 109, A10304, doi:10.1029/2004JA010607, 2004.

Fritz, H.: Das Polarlicht, Brockhaus, Leipzig, 348 pp., 1881.

Fukunishi, N.: Constitution of proton and electron auroral substorms. 2. Dynamical morphology of proton aurora at auroral substorms and phenomenological model for magnetospheric substorm, Tech. rep., Jap. Antarctic Res. Exped. Sci. Rep., 1973.

Fukunishi, N.: Dynamic relationship between proton and electron auroral substorms, J. Geophys. Res., 80, 553-574, 1975.

Fuller, V. B.: A report on the aurora borealis for the year 1932-1934, Terr. Magn. Atmos. Elect., 40, 269-275, 1935.

Galperin, Y. I.: Hydrogen emissions and two types of auroral spectra, Planet. Space Sci., 1, 57-62, 1959.

Galperin, Y. I.: Proton bombardment in aurora, Planet. Space Sci., 10, 187-193, 1963.

Galperin, Y. I. and Feldstein, Y. I.: Mapping of the precipitation regions to the plasma sheet, J. Geomag. Geoelectr., 48, 857-875, 1996.

Galperin, Y. I., Ponomarev, V. N., and Zosimova, A. G.: Plasma convection in the polar ionosphere, Ann. Geophys., 30, 1-7, 1974, http://www.ann-geophys.net/30/1/1974/.

Galperin, Y. I., Crasnier, J., Lissakov, Y. V., Nikolaenko, L. M., Sinitsin, V. M., Sauvaud, J. A., and Khalipov, V. L.: Diffuse auroral zone, I. A model of the equatorial border of the auroral electron diffuse precipitation zone in the evening and near-midnight sectors, Kosm. Issled., 15, 421-434, 1977 (in Russian).

Gartlein, C. W.: Auroral spectra showing broad hydrogen lines, EOS Trans. AGU, 31, 18-20, 1950.

Gjerloev, J. W., Hoffman, R. A., Sigwarth, J., and Frank, L. A.: Statistical description of the bulge-type auroral substorm in the far ultraviolet, J. Geophys. Res., 112, A07213, doi:10.1029/2006JA012189, 2007.

Gjerloev, J. W., Hoffman, R. A., Sigwarth, J., Frank, L. A., and Baker, J. B. H.: Typical auroral substorm: A bifurcated oval, J. Geophys. Res., 113, A03211, doi:10.1029/2007JA012431, 2008. 
Gusev, M. G. and Troshichev, O. A.: Relation of sun-aligned arcs to polar cap convection and magnetic disturbances, Planet. Space Sci., 38, 1-11, 1990.

Gussenhoven, M. S.: Extremely high latitude auroras, J. Geophys. Res., 87, 2401-2412, 1982.

Gussenhoven, M. S., Hardy, D. A., and Burke, W. J.: DMSP/F2 electron observations of equatorward auroral boundaries and their relationship to magnetospheric electric fields, J. Geophys. Res., 86, 768-778, 1981.

Gustafsson, G., Feldstein, Y. I., and Starkov, G. V.: The auroral orientation curves for the iqsy, Planet. Space Sci., 17, 1657-1667, 1969.

Harang, L.: The mean field of disturbance of polar geomagnetic storms, Terr. Magn. Atmos. Elect., 51, 353-380, 1946.

Hardy, D. A., Burke, W. J., Gussenhoven, M. S., Heinemann, N., and Holeman, E.: DMSP/F2 electron observations of equatorward auroral boundaries and their relationship to the solar wind velocity and the north-south component of the interplanetary magnetic field, J. Geophys. Res., 86, 9961-9974, 1981.

Heikkila, W. J. and Winningham, J. D.: Penetration of magnetosheath plasma to low altitudes through the dayside magnetospheric cusps, J. Geophys. Res., 76, 883-891, 1971.

Heikkila, W. J., Winningham, J. D., Eather, R. H., and Akasofu, S.I.: Auroral emission and particle precipitation in the noon sector, J. Geophys. Res., 77, 4100-4115, 1972.

Henderson, M. G., and Murphree, J. S.: Comparison of Viking onset location with predictions of the thermal catastrophe model, J. Geophys. Res., 100, 21857-21872, 1995.

Henderson, M. G., Murphree, J. S., and Weygand, J. M.: Observation of auroral substorms occurring together with preexisting "quiet time" auroral patterns, J. Geophys. Res., 101, 2462124640, 1996.

Henderson, M. G., Reeves, G. D., Skoug, R., Thomsen, M. F., Denton, M. H., Mende, S. B., Immel, T. J., Brandt, P. C., and Singer, H. J.: Magnetospheric and auroral activity during the 18 April 2002 sawtooth event, J. Geophys. Res., 111, A01S90, doi:10.1029/2005JA011111, 2006a.

Henderson, M. G., Skoug, R., Donovan, E., Thomsen, M. F., Reeves, G. D., Denton, M. H., Singer, H. J., McPherron, R. L., Mende, S. B., Immel, T. J., Sigwarth, J. B., and Frank, L. A.: Substorms during the 10-11 August 2000 sawtooth event, J. Geophys. Res., 111, A06206, doi:10.1029/2005JA011366, 2006 b.

Henderson, M. G., Donovan, E., Foster, J. C., Mann, I., Immel, T. J., Mende, S. B., and Sigwarth, J. B.: Start-to-end global imaging of a sunward propagating, SAPS-associated giant undulation event, J. Geophys. Res., 115, A04210, doi:10.1029/2009JA014106, 2010.

Heppner, J. P.: Time sequences and spatial relations in auroral activity during magnetic bays at College, Alaska, J. Geophys. Res., 59, 329-338, 1954.

Hoffman, R. A., Gjerloev, J. W., Frank, L. A., and Sigwarth, J. W.: Are there optical differences between storm-time substorms and isolated substorms?, Ann. Geophys., 28, 1183-1198, doi:10.5194/angeo-28-1183-2010, 2010.

Holzworth, R. H. and Meng, C.-I.: Mathematical representation of the auroral oval, Geophys. Res. Lett., 2, 377-380, 1975.

Horwitz, J. L. and Akasofu, S.-I.: The response of the dayside aurora to sharp northward and southward transition of the inteplan- etary magnetic field and to magnetospheric substorm, J. Geophys. Res., 82, 2723-2734, 1977.

Hulburt, E. O.: On the diurnal variation of the aurora polaris, Terr. Magn. Atmos. Elect., 36, 23-40, 1931.

Hultqvist, B.: The geomagnetic field lines in higher approximations, Arkiv Geofysik, 3, 63-77, 1958.

Hultqvist, B.: Auroral isochasms, Nature, 183, 1478-1480, 1959.

Hultqvist, B.: On the orientation of auroral arcs, J. Atmos. Terr. Phys., 24, 17-30, 1962.

Hultqvist, B., Egeland, A., and Gustafsson, G.: A morning discontinuity in the orientation of quiet auroral arcs, Nature, 192, 956957, doi:10.1038/192956a0, 1961.

Ievenko, I. B.: Dynamics of the diffusive auroral luminescence and the SAR-arcs during substorms, Geomagn. Aeron., 33, 42-57, 1993 (in Russian).

Ievenko, I. B.: Influence of the magnetospheric activity on the plasmasphere according to observations of diffusive luminescence and SAR-arcs, Geomagn. Aeron., 39, 26-32, 1999 (in Russian).

Ievenko, I. B. and Alexeev, V. N.: Influence of substorms and storms on the dynamics of SAR-arcs, Statistical analysis, Geomagn. Aeron., 44, 643-654, 2004 (in Russian).

Iijima, T. and Nagata, T.: Signatures for substorm development of the growth phase and expansion phase, Planet. Space Sci., 20, 1095-1098, 1972.

International Auroral Atlas (1963), International Auroral Atlas, Edinburgh Univ. Press, Edinburgh, 1963.

Isaev, S. I.: About diurnal variation of the auroral activity, Problems of the Arctic, 1, 27-37, 1939a (in Russian).

Isaev, S. I.: Some pecularities in the appearance of aurorae in different zones, Problems of the Arctic, 1, 21-30, 1939b (in Russian).

Isaev, S. I.: About diurnal periodicity of auroras inside the zone of maximal frequency, Problems of the Arctic, 2, 41-45, 1940 (in Russian).

Isaev, S. I.: Geographical distribution of aurora and the Earth's radiation belts, Geomagn. Aeron., 2, 663-668, 1962 (in Russian).

Isaev, S. I.: New International Auroral Atlas, 95-98, Publ. House Academy Sci. USSR, Moscow, 1964.

Ismail, S., Wallis, J. A., and Cogger, L. L.: Characteristics of polar cap sun-aligned arcs, J. Geophys. Res., 82, 4741-4749, 1977.

Ivliev, D. Y., Pudovkin, M. I., and Zaitseva, S. A.: Development of an elementary magnetic disturbance, Geomagn. Aeron., 10, 300304, 1970 (in Russian).

Judge, R. J. R.: Electron excitation and auroral emission parameters, Planet. Space Sci., 20, 2081-2092, 1972.

Kamide, Y., and Winningham, J. D.: A statistical study of the "instantaneous" nightside auroral oval: The equatorward boundary of electron precipitation as observed by the Isis 1 and 2 satellites, J. Geophys. Res., 82, 5573-5588, 1977.

Kan, J. R. and Lee, L. C.: Energy coupling function and solar windmagnetosphere dynamo, Geophys. Res. Lett., 6, 577-580, 1979.

Kelley, M. C.: Intense sheared flow as the origin of large-scale undulations of the edge of the diffuse aurora, J. Geophys. Res., 91, 3225-3230, 1986.

Khorosheva, O.: On the space-time distribution of auroras in the arctic during the winter 1957-1958, in: Transactions "Aurora and Airglow", Vol. 7, 14-21, USSR Academy of Science Publishing House, Moscow, 1961. 
Khorosheva, O. and Emelyanenko, S. N.: Altitudinal asymmetry of the instantaneous auroral zone, Geomagn. Aeron., 9, 461-463, 1970.

King, J. H.: Interplanetary medium data book, Vol. 77-04a, Greenbelt, 1977.

Kisabeth, J. L. and Rostoker, G.: Development of the polar electrojet during polar magnetic substorms, J. Geophys. Res., 76, 68156828, 1971.

Kornilov, I. A., Kornilova, T. A., and Kornilov, O. I.: Some aspects of individual and multiple polar arcs generation, in: Proceedings of the 33rd Annual European Meeting on Atmospheric Studies by Optical Methods, vol. 292, 111-118, Sci. Report IRF, 2008.

Kornilov, T. A. and Kornilova, I. A.: Spatial-temporal dynamics of auroras during magnetic storms, Geomagn. Aeron., 49, 722-732, 2009.

Kornilova, T. A. and Kornilov, I. A.: Counterstreaming auroral structures during substorm expansion, J. Geophys. Res., 117, A05328, doi:10.1029/2011JA017309, 2012.

Kornilova, T. A., Kornilov, I. A., and Kornilov, O. I.: Auroral intensification structure and dynamics in the double oval: the substorm of December 26, 2000, Geomagn. Aeron., 46, 450-456, 2006.

Kornilova, T. A., Kornilov, I. A., and Kornilov, O. I.: Fine structure of breakup development inferred from satellite and ground-based observations, Ann. Geophys., 26, 1141-1148, doi:10.5194/angeo-26-1141-2008, 2008.

Koskinen, H. E. J., Lopez, R. E., Pulkkinen, T. I., Baker, D. N., and Bösinger, T.: Pseudobreakup and substorm growth phase in the ionosphere and magnetosphere, J. Geophys. Res., 93, 58015813, 1993.

Krasovsky, V. I.: Aurorae, Geomagn. Aeron., 7, 945-957, 1967.

Krasovsky, V. I., Shefov, N. N., and Yarin, V. I.: Atlas of the airglow spectrum 3000-12400 Å, Planet. Space Sci., 9, 833-915, 1962.

Lanzerotti, L. J., Hasegawa, A., and Maclennan, C. J.: Hydromagnetic waves as a cause of a SAR arc event, Planet. Space Sci., 26, 777-783, 1978.

Lassen, K.: Existence of an inner auroral zone, Nature, 184, 13751377, 1959.

Lassen, K.: Geographical distribution and temporal variations of polar aurorae, in: Meddelelser, Vol. 16, Det Danske Meteor. Inst. Meddelelser, Charlottenlund, 1963.

Lassen, K.: On the classification of high-latitude auroras, Geofysiske Publikasjoner, 29, 87-104, 1972.

Lassen, K. and Danielsen, C.: Quiet time pattern of auroral arcs for different direction of interplanetary magnetic field in the Y-Z plane, J. Geophys. Res., 83, 5277-5284, 1978.

Lassen, K., Sharber, J. R., and Winningham, J. D.: The development of auroral and geomagnetic substorm activity after a southward turning of the interplanetary magnetic field following several hours of magnetic calm, J. Geophys. Res., 82, 5031-5050, 1977.

Lassen, K., Danielsen, C., and Meng, C.-I.: Average configuration of the quiet auroral oval, in: Geophys. Papers, Vol. R-73, Danish Meteorol. Inst., 29 pp., 1986.

Lee, D.-Y., Choi, K.-C., Ohtani, S., Lee, J. H., Kim, K. C., Park, K. S., and Kim, K.-H.: Can intense substorms occur under northward IMF conditions?, J. Geophys. Res., 115, A01211, doi:10.1029/2009JA014480, 2010.
Leontyev, S. V., Starkov, G. V., Vorobjev, V. G., Zverev, V. L., and Feldstein, Y. I.: Dayside aurorae and their relation to other geophysical phenomena, Planet. Space Sci., 40, 621-639, 1992.

Lezniak, T. W. and Winckler, J. R.: Experimental study of magnetospheric motions and the acceleration of energetic electrons during substorms, J. Geophys. Res., 75, 7075-7098, 1970.

Liou, K.: Polar Ultraviolet Imager observation of auroral breakup, J. Geophys. Res., 115, A12219, doi:10.1029/2010JA015578, 2010.

Lomonosov, M. V.: Works on Physics 1753-1756 of M. V. Lomonosov - The entire collection, Academy Sci. USSR, Moscow - Leningrad, 604 pp., 1952 (in Russian).

Loomis, E.: On geographical distribution of auroras in northern hemisphere, Amer. J. Sciences, 30, 89-94, 1860.

Lui, A. T. Y. and Anger, C. D.: A uniform belt of diffuse auroral emission seen by the ISIS-2 scanning photometer, Planet. Space Sci., 21, 799-809, 1973.

Lui, A. T. Y., Perreault, P., Akasofu, S.-I., and Anger, C. D.: The diffuse aurora, Planet. Space Sci., 21, 857-861, 1973.

Lui, A. T. Y., Anger, C. D., and Akasofu, S.-I.: The equatorial boundary of the diffuse aurora and auroral substorms as seen by the ISIS-2 auroral scanning photometer, J. Geophys. Res., 80, 3603-3614, 1975.

Lui, A. T. Y., Venkatesan, D., and Anger, C. D.: Simultaneous observations of particle precipitations and auroral emissions by ISIS-2 satellite in the 19-24 MLT-sector, J. Geophys. Res., 82, 22102226, 1977.

Lui, A. T. Y., Meng, C.-I., and Ismail, S.: Large amplitude undulations on the equatorward boundary of diffuse aurora, J. Geophys. Res., 87, 2385-2400, 1982.

Lundblad, J. A. and Søraas, F.: Proton observations supporting the ion cyclotron wave heating theory of SAR arc formation, Planet. Space Sci., 26, 245-254, 1978.

Makita, K., Meng, C.-I., and Akasofu, S.-I.: Transpolar auroras, their particle precipitation, and IMF $B_{y}$-component, J. Geophys. Res., 96, 14085-14095, 1991.

Marovich, E. and Roach, F. E.: Distribution of latitudes of red arcs, J. Geophys. Res., 68, 1885-1888, 1963.

Mawson, D.: Auroral observations at the Cape Royds Station, Antarctica, T. Roy. Soc. South Aust., XL, 151-212, 1916.

McDiarmid, I. B., Burrows, J. R., and Budzinski, E. E.: Average characteristics of magnetospheric electrons $(150 \mathrm{eV}$ to $200 \mathrm{keV})$ at $1400 \mathrm{~km}$, J. Geophys. Res., 80, 73-79, 1975.

McPherron, R. L.: Growth phase of magnetospheric substorms, J. Geophys. Res., 75, 5592-5599, 1970.

Meinel, A. B.: On the entry into Earth's atmosphere of 57-kev protons during auroral activity, Phys. Rev., 80, 1096-1097, 1950.

Mende, S. B. and Eather, R. H.: Monochromatic all-sky observations and auroral precipitation patterns, J. Geophys. Res., 81, 3771-3780, 1976.

Meng, C.-I.: Electron precipitations and polar auroras, Space Sci. Rev., 22, 223-300, 1978.

Meng, C.-I.: Electron precipitation in the midday auroral oval, J. Geophys. Res., 86, 2149-2174, 1981.

Meng, C.-I. and Lundin, R.: Auroral morphology of the midday oval, J. Geophys. Res., 91, 1572-1584, 1986.

Meng, C.-I., Holzworth, R. H., and Akasofu, S.-I.: Auroral circle - delineating the poleward boundary of the quiet auroral belt, J. Geophys. Res., 82, 164-172, 1977. 
Murphree, J. S., Anger, C. D., and Cogger, L. L.: The instantaneous relationship between polar cap and oval auroras at times of northward interplanetary magnetic field, Can. J. Phys., 60, 349-356, 1982.

Nagata, T., Hirasava, T., and Agukawa, M.: Discrete and diffuse auroral belts in Antarctica, Rep. Ionosph. Space Res. Jap., 29, 149-156, 1975.

Nakai, H. and Kamide, Y.: Response of nightside auroral oval boundaries to the interplanetary magnetic field, J. Geophys. Res., 88, 4005-4014, 1983.

Nakamura, R., Baker, D. N., Yamamoto, T., Belian, R. D., Bering III, E. A., Benbrook, J. R., and Theall, J. R.: Particle and field signatures during pseudobreakup and major expansion onset, $\mathrm{J}$. Geophys. Res., 99, 207-221, 1994.

Newell, P. T., Sotirelis, T., Liou, K., Meng, C.-I., and Rich, F. J.: A nearly universal solar wind-magnetosphere coupling function inferred from 10 magnetospheric state variables, J. Geophys. Res., 112, A01206, doi:10.1029/2006JA012015, 2007.

Newell, P. T., Liou, K., and Wilson, G. R.: Polar cap particle precipitation and aurora: Review and commentary, J. Atmos. Sol.-Terr. Phy., 71, 199-215, 2009.

Nikolsky, A. P.: Photography of polar lights in the Tikhaya Bay, Problems of the Arctic, No. 10-11, 131-133, Publ. House Glavsevmorputi, Leningrad, 1939 (in Russian).

Nikolsky, A. P.: On the second zone of magnetic disturbances intensity increased in near-polar region, in: Transactions of the Arctic and Antarctic Institute, vol. 83, 5-83, AARI, Leningrad, USSR, 1956.

Nikolsky, A. P.: On the issue of geographical distribution of auroras in the Arctic, in: Transactions "Investigation of aurorae", vol. 4, 14-19, USSR Academy of Science Publishing House, Moscow, 1960.

Nishida, A.: Formation of the plasmapause, or magnetospheric plasma knee, by the combined action of magnetospheric convection and plasma escape from the tail, J. Geophys. Res., 71, 5669-5679, 1966.

Nishimura, Y., Lyons, L., Zou, S., Angelopoulos, V., and Mende, S.: Substorm triggering by new plasma intrusion: THEMIS all-sky imager observations, J. Geophys. Res., 115, A07222, doi:10.1029/2009JA015166, 2010.

Nishitani, N., Hough, G., and Scourfield, M. W. J.: Spatial and temporal characteristics of giant undulations, Geophys. Res. Lett., 21, 2673-2676, 1994.

Nordenskiöld, A. E.: Vegas fard kring Asien ach Europa, F. G. Beijer, Stockholm, 1881.

O‘Brien, B. J.: A large diurnal variation of the geomagnatically trapped radiation, J. Geophys. Res., 68, 989-997, 1963.

Oguti, T.: Hydrogen emission and electron aurora at the onset of the auroral breakup, J. Geophys. Res., 78, 7543-7547, 1973.

Oguti, T.: Metamorphoses of aurora, Memoirs Nat. Inst. Polar Res. Japan, Series A, 1-101, 1975.

Omholt, A., Stroffregan, W., and Derblom, H.: Hydrogen lines in auroral glow, J. Atmos. Terr. Phys., 24, 203-209, 1962.

Paschmann, G., Haaland, S., and Treumann, R. (Eds.), Auroral Plasma Physics, Space Sciences Series of ISSI (SSSI), vol. 15, Kluwer Academic Publishers, Dordrecht, Boston, London, reprinted from Space Science Reviews Vol. 103, No. 1-4, 2002 .
Perreault, P. and Akasofu, S.-I.: A study of geomagnetic storms, Geophys. J. Roy. Astr. S., 54, 547-573, 1978.

Pike, C. P. and Dandekar, B. S.: Evening sector auroral oval dynamics from DMSP photographs, J. Geophys. Res., 84, 3389-3402, 1979.

Pike, C. P. and Whalen, J. A.: Satellite observations of auroral substorms, J. Geophys. Res., 79, 985-1000, 1974.

Pudovkin, M. I., Semenov, V. S., Starkov, G. V., and Kornilova, T. A.: On separation of the potential and vortex parts of the magnetotail electric field, Planet. Space Sci., 39, 563-568, 1991.

Pushkov, N. V.: Observations of the aurorae. Instructions., Publ. House Nauka, USSR, Moscow, 1957.

Pytte, T., McPherron, R. L., Kivelson, M. G., West Jr., H. I., and Hones Jr., E. W.: Multiple-satellite studies of magnetospheric substorms - radial dynamics of the plasma sheet, J. Geophys. Res., 81, 5921-5933, 1976a.

Pytte, T., McPherron, R. L., and Kokubun, S.: The ground signatures of the expansion phase during multiple onset substorms, Planet. Space Sci., 24, 1115-1132, 1976 b.

Pytte, T., McPherron, R. L., Hones Jr., E. W., and West Jr., H. I.: Multiple-satellite studies of magnetospheric substorms: Distinction between polar magnetic substorms and convection-driven negative bays, J. Geophys. Res., 83, 663-679, 1978.

Rae, I. J., Mann, I. R., Angelopoulos, V., Murphy, K. R., Milling, D. K., Kale, A., Frey, H. U., Rostoker, G., Russell, C. T., Watt, C. E. J., Engbretson, M. J., Moldwin, M. B., Mende, S. B., Singer, H. J., and Donovan, E. F.: Near-Earth initiation of a terrestrial substorm, J. Geophys. Res., 114, A07220, doi:10.1029/2008JA013771, 2009.

Rae, I. J., Watt, C. E. J., Mann, I. R., Murphy, K. R., Samson, J. C., Kabin, K., and Angelopoulos, V.: Optical characterization of the growth and spatial structure of a substorm onset arc, J. Geophys. Res., 115, A10222, doi:10.1029/2010JA015376, 2010.

Rassoul, H. K., Rohrbaurgh, R. P., Tinsley, B. A., and Slater, D. W.: Spectrometric and photometric observations of low-latitude aurorae, J. Geophys. Res., 98, 7695-7709, 1993.

Rees, M. H. and Akasofu, S.-I.: On the association between subvisual red arcs and the Dst $(\mathrm{H})$ decrease, Planet. Space Sci., 11, 105-107, 1963.

Rees, M. H. and Benedict, P. C.: Auroral proton oval, J. Geophys. Res., 75, 4763-4774, 1970.

Rees, M. H. and Luckey, D. J.: Auroral electron energy derived from ratio of spectroscopic emissions. 1. Model computation, J. Geophys. Res., 79, 5181-5186, 1974.

Rees, M. H. and Roble, R. G.: Observations and theory of the formation of stable auroral red arcs, Rev. Geophys., 13, 201-242, 1975.

Rees, M. H., Belon, A. E., and Romick, G. J.: The systematic behaviour of hydrogen emission in the aurora - I, Planet. Space Sci., 5, 87-91, 1961.

Reid, G. C. and Rees, M. H.: The systematic behaviour of hydrogen emission in the aurora - II, Planet. Space Sci., 5, 99-105, 1961.

Roach, F. E. and Roach, J. R.: Stable 6300å auroral arcs in mid latitudes, Planet. Space Sci., 11, 523-545, 1963.

Rodrigues, J. V., Valladares, C. E., Fukui, K., and Callagher, H. A.: Antisunward decay of polar cap arcs, J. Geophys. Res., 102, 27227-27248, 1992.

Rostoker, G.: Current flow in the magnetosphere during magnetospheric substorms, J. Geophys. Res., 79, 1994-1998, 1974. 
Rostoker, G. and Camide, F. P.: Localized character of magnetotail magnetic fluctuations during polar magnetic substorms, J. Geophys. Res., 76, 6944-6951, 1971.

Rostoker, G. and Fälthammar, C.-G.: Relationship between changes in the interplanetary magnetic field and variations in the magnetic field at the Earth's surface, J. Geophys. Res., 72, 5853-5863, 1967.

Rostoker, G., Akasofu, S.-I., Foster, J., Greenwald, R. A., Kamide, Y., Kawasaki, K., Lui, A. T. Y., McPherron, R. L., and Russell, C. T.: Magnetospheric substorms - definition and signatures, J. Geophys. Res., 85, 1663-1668, 1980.

Sandford, B. P.: Aurora and airglow intensity variations with time and magnetic activity at southern high latitudes, J. Atmos. Terr. Phys., 26, 749-769, 1964.

Sandford, B. P.: Variations of auroral emissions with time, magnetic activity and the solar cycle, J. Atmos. Terr. Phys., 30, 1921-1942, 1968.

Sandholt, P. E. and Farrugia, C. J.: Monitoring magnetosheathmagnetosphere interconnection topology from the aurora, Ann. Geophys., 20, 629-637, doi:10.5194/angeo-20-629-2002, 2002.

Sandholt, P. E. and Farrugia, C. J.: Poleward moving auroral forms (PMAFs) revisited: responses of aurorae, plasma convection and Birkeland currents in the pre- and postnoon sectors under positive and negative IMF $B_{y}$ conditions, Ann. Geophys., 25, 16291652, doi:10.5194/angeo-25-1629-2007, 2007.

Sandholt, P. E., Egeland, A., Lybekk, B., Deehr, C. S., Sivjee, G. G., and Romick, G. J.: Effect of interplanetary magnetic field and magnetospheric substorm variations on the dayside aurora, Planet. Space Sci., 31, 1345-1362, 1983.

Sandholt, P. E., Egeland, A., Holtet, J. A., Libekk, B., Svenes, K., Asheim, S., and Deehr, C. S.: Large- and small-scale dynamics of polar cusp, J. Geophys. Res., 90, 4407-4414, 1985.

Sandholt, P. E., Egeland, A., Deehr, C. S., Vorobjev, V. G., Starkov, G. V., Zverev, V. L., and Feldstein, Y. I.: Dayside auroral luminosity in relation to IMF and magnetospheric substorm activity, electrodynamical processes in high latitudes, in: Proceedings International Symposium on Polar geomagnetic phenomena, 25-31 May 1986, Suzdal, Russia, 116-145, Apatity, Russian Academy of Science, 1988.

Sandholt, P. E., Moen, J., Rudland, A., Opsvik, D., Dening, W. E., and Hansen, T.: Auroral event sequences at the dayside polar cap boundary for positive and negative interplanetary magnetic field $B_{y}$, J. Geophys. Res., 98, 7737-7755, 1993.

Sandholt, P. E., Farrugia, C. J., Moen, J., Norberg, O., Lybekk, B., Sten, T., and Hansen, T.: A classification of dayside auroral forms and activities as a function of IMF orientation, J. Geophys. Res., 103, 23325-23345, 1998.

Sandholt, P. E., Carlson, C., and Egeland, A.: Dayside and polar cap aurora, Astrophys. Space Sci. Library, Vol. 270, Kluwer Academ. Pub., Dordrecht/Boston/London, 287 pp., 2002a.

Sandholt, P. E., Farrugia, C. J., Lester, M., Cowley, S., Milan, S., Denig, W. F., Lybekk, B., Trondsen, E., and Vorobjev, V. G.: Multistage substorm expansion: Auroral dynamics in relation to plasma sheet particle injection, precipitation, and plasma convection, J. Geophys. Res., 107, 1342, doi:10.1029/2001JA900116, 2002b.

Sauvaud, J. A., Jacquey, C., Oka, M., Palin, L., Fruit, G., Kistler, L. M., Balogh, A., Cao, J. B., Reeves, G., Mukai, T., Shinohara, I., and Grigorenko, E.: A study of the changes of the near-Earth plasma sheet and lobe driven by multiple substorms: Comparison with a full particle simulation of reconnection, J. Geophys. Res., 117, A01221, doi:10.1029/2011JA017033, 2012.

Sergeev, V. A.: On the longitudinal localization of the substorm active region and its changes during the substorm, Planet. Space Sci., 22, 1341-1343, 1974

Sergeev, V. A.: On the state of the magnetosphere during prolonged period of southward oriented IMF, Physica Solariterrestris, Potsdam, 5, 39-44, 1977a.

Sergeev, V. A.: On the microstructure of the magnetospheric substorms, Geomag. Res., 21, 5-15, 1977b (in Russian).

Sergeev, V. A. and Vorobjev, V. G.: Structure of aurorae in the period of developed stationary convection, Geomag. Res., 25, 60-68, 1979 (in Russian).

Sergeev, V. A. and Yahnin, A. G.: Motion of polar-cap auroral arcs, Geomagn. Aeron., 18, 744-745, 1978.

Sergeev, V. A. and Yahnin, A. G.: A correspondence of substorm expansive phase signatures, Geomag. Res., 24, 78-89, 1979a (in Russian).

Sergeev, V. A. and Yahnin, A. G.: Detailed study of microsubstorm beginnings, Geomag. Res., 24, 90-100, 1979b (in Russian).

Sergeev, V. A. and Yahnin, A. G.: The features of auroral bulge expansion, Planet. Space Sci., 27, 1429-1440, 1979c.

Sergeev, V. A., Liou, K., Meng, C.-I., Newell, P. T., Brittnacher, M., Parks, G., and Reeves, G. D.: Development of auroral streamers in association with localized impulsive injections to the inner magnetotail, Geophys. Res. Lett., 26, 417-420, doi:10.1029/1998GL900311, 1999.

Sergeev, V. A., Liou, K., Newell, P. T., Ohtani, S.-I., Hairston, M. R., and Rich, F.: Auroral streamers: characteristics of associated precipitation, convection and field-aligned currents, Ann. Geophys., 22, 537-548, doi:10.5194/angeo-22-537-2004, 2004.

Sergeev, V. A., Nikolaev, A. V., Tsyganenko, N. A., Angelopoulos, V., Runov, A. V., Singer, H. J., and Yang, J.: Testing a two-loop pattern of the substorm current wedge (SCW2L), J. Geophys. Res., 119, 947-963, doi:10.1002/2013JA019629, 2014.

Shepherd, G. G., Thirkettle, F. W., and Anger, C. D.: Topside optical view of the dayside cleft aurora, Planet. Space Sci., 24, 937-944, 1976.

Shue, J.-H., Newell, P. T, Liou, K., Meng, C.-I., Kamide, Y., and Lepping, R. P.: Two-component auroras, Geophys. Res. Lett., 29, 1379, doi:10.1029/2002GL014657, 2002.

Shue, J.-H., Newell, P. T., Liou, K., Meng, C.-I., Hairston, M. R., and Rich, F. J.: Ionospheric characteristics of the dusk-side branch of the two-cell aurora, Ann. Geophys., 24, 203-214, doi:10.5194/angeo-24-203-2006, 2006.

Slater, D. W., Smith, L. L., and Kleckner, E. W.: Correlated observations of the equatorward diffuse auroral boundary, J. Geophys Res., 85, 531-542, 1980.

Slater, D. W., Gurgiolo, C., Kozyra, J. U., Kleckner, E. W., and Winningham, J. D.: A possible energy source to power stable auroral red arcs: Precipitating electrons, J. Geophys. Res., 92, 45434552, 1987.

Snyder, A. L. and Akasofu, S.-I.: Observations of the auroral oval photographs by the Alaskan meridian chain of stations, J. Geophys. Res., 77, 3419-3430, 1972.

Snyder, A. L. and Akasofu, S.-I.: Major auroral substorm features in the dark sector observed by a USAF DMSP satellite, Planet. Space Sci., 22, 1511-1517, 1974. 
Snyder, A. L. and Akasofu, S.-I.: Auroral oval photographs from DMSP 8531 and 10533 satellites, J. Geophys. Res., 81, 17991804, 1976.

Starkov, G.: Planetary morphology of auroras, Publ. House Nauka, St.-Peterburg, 184 pp., 1993 (in Russian).

Starkov, G.: Mathematical description of auroral luminosity boundaries), Geomagn. Aeron., 34, 80-86, 1994 (in Russian.

Starkov, G. V.: Auroral heights in the polar cap, Geomagn. Aeron., 8, 28-33, 1968 (in Russian).

Starkov, G. V. and Feldstein, Y. I.: The azimuths of auroral arcs according to observations at the Dixon Island, in: IGY Transactions "Auroras and Airglow", Vol. 4, 56-61, Publ. House Academy Sci. USSR, 1960 (in Russian).

Starkov, G. V. and Feldstein, Y. I.: Orientations of elongated auroral forms, Geomagn. Aeron., 7, 72-77, 1967a (in Russian).

Starkov, G. V. and Feldstein, Y. I.: Scheme of elementary disturbance in aurorae on the dayside of the Earth, Geomagn. Aeron., 7, 367-369, 1967b (in Russian).

Starkov, G. V. and Feldstein, Y. I.: Instantaneous distribution of auroras and polar geomagnetic disturbances, in Transactions "Auroras", Vol. N19, 32-41, USSR Academy of Science Publishing House, 1970.

Starkov, G. V. and Feldstein, Y. I.: Substorms in the auroras, Geomagn. Aeron., 11, 563-565, 1971a.

Starkov, G. V. and Feldstein, Y. I.: Substorms in auroras, Geomagn. Aeron., 11, 560-562, 1971b (in Russian).

Starkov, G. V., Feldstein, Y. I., and Shevnina, N. F.: Movement of auroral forms during the development of substorms, 53-67, Publ. House Nauka, USSR, Leningrad, 1971 (in Russian).

Starkov, G. V., Feldstein, Y. I., and Shevnina, N. F.: Dayside aurorae during substorms, Geomagn. Aeron., 13, 86-90, 1973 (in Russian).

Størmer, C.: Résultats des mesures photogrammétiques des aurores boreales observées dans la Norvège méridionale de 1911 à 1922, Geofysiske Publikasjoner, 4, 1-157, 1927.

Størmer, C.: The Polar Aurora, Clarendon Press, 402 pp., 1955.

Strickland, D. J., Jasperse, J. R., and Whalen, J. A.: Dependence of auroral FUV emissions on the incident electron spectrum and neutral atmosphere, J. Geophys. Res., 88, 8051-8062, 1983.

Stringer, W. J., Belon, A. E., and Akasofu, S.-I.: The latitude of aurora1 activity during periods of zero and very weak magnetic disturbance, J. Atmos. Terr. Phys., 27, 1039-1044, 1965.

Swjatskij, D.: Aurora borealis in the Russian literature and science from the X-th to the XVIII-th century, Archive of Science and Technique History, 1, 47-67, 1934 (in Russian).

Sykora, J.: Die Wellenlängen der photographisch erhaltenen Linien des Nordlichtspectrums, Astron. Nachr., 156, 325-326, 1901.

Tromholt, S.: Sur les periodes de l'aurore boreale, Dan. Meteor. Inst. Copenhagen, 1882.

Troshichev, O. A. and Gusev, S. V.: IMF $B_{x}$ and $B_{y}$ dependence of the polar cap auroral distribution for northward IMF orientation inferred from observations at Vostok station, J. Atmos. Terr. Phys., 56, 237-244, 1994.

Troshichev, O. A., Andrezen, V. G., Vennerstrom, V. G., and FriisChristensen, E.: Magnetic activity in the polar cap - A new index, Planet. Space Sci., 36, 1095-1102, 1988.

Tsurutani, B., Zhou, X.-Y., and Gonzalez, W. D.: A lack of substorm expansion phases during magnetic storms induced by magnetic clouds, in: Disturbances in Geospace: The Storm-Substorm Rela- tionship, Geophysical Monograph, Vol. 142, edited by: Sharma, A. S., Kamide, Y., and Lakhina, G. S., 23-36, American Geophysical Union, 2003.

Valladares, C. E., Carlson Jr., H. C., and Fukui, K.: Interplanetary magnetic field dependency of stable sun-aligned polar cap arcs, J. Geophys. Res., 99, 6247-6272, 1994.

Vegard, L.: On the properties of the rays producing aurorae borealis, Philos. Mag., 23, 211-237, 1912.

Vegard, L.: Hydrogen showers in the auroral region, Nature, 144, 1089-1090, 1939.

Vegard, L.: An aurora spectrogram obtained in Oslo on February 23, Nature, 165, 1012-1013, 1950.

Vegard, L. and Krogness, O.: The position in space of the aurora polaris, Geofysiske Publikasjoner, 1, 1-227, 1920.

Vestine, E. H.: The geographic incidence of aurora and magnetic disturbance. Northern hemisphere, Terr. Magn. Atmos. Elect., 49, 77-102, 1944.

Vestine, E. H. and Snyder, E. J.: The geographic incidence of aurora and magnetic disturbance. Southern hemisphere, Terr. Magn. Atmos. Elect., 50, 105-124, 1945.

Viñas, A. F. and Madden, T. R.: Share flow-ballooning instability as a possible mechanism for hydrodynamic fluctuations, J. Geophys. Res., 91, 1519-1528, 1986.

Vorobjev, V. G. and Rezhenov, B. V.: Step-like westward displacement of the auroral substorm localization during impulsive enhancements of the magnetic field, in: Transactions "Substorms and disturbances in the magnetosphere", 103-115, Publ. House Nauka, Leningrad, USSR, 1975 (in Russian).

Vorobjev, V. G. and Rezhenov, B. V.: Characteristics of dayside discrete auroral gap, in Transactions "Auroras", vol. N30, 71-76, USSR Academy of Science Publishing House, 1982 (in Russian).

Vorobjev, V. G. and Turjansky, V. A.: Peculiarities of the behavior of emissions (oi) $\lambda 6300$ å and $\lambda 5577 \AA$ in the daytime auroras, Geomagn. Aeron., 23, 957-962, 1983 (in Russian).

Vorobjev, V. G. and Zverev, V. I.: Polar cap aurora intensifications and their relation to the solar wind, in Proceedings of the 33rd Annual European Meeting on Atmospheric Studies by Optical Methods, vol. A-2, Kiljavanranta Training Center, Finland, 1995.

Vorobjev, V. G., Gustafsson, G., Starkov, G. V., Feldstein, Y. I., and Shevnina, N.: Dynamics of day and night aurora during substorms, Planet. Space Sci., 23, 269-278, 1975.

Vorobjev, V. G., Rezhenov, B. V., and Starkov, G. V.: Relation between the location of eastern electrojet, the boundary of energetic electrons capture and aurorae, Geomagn. Aeron., 16, 304-310, 1976 a.

Vorobjev, V. G., Starkov, G. V., and Feldstein, Y. I.: The auroral oval during the substorm development, Planet. Space Sci., 24, 955-965, 1976b.

Vorobjev, V. G., Zverev, V. L., and Leontyev, S. V.: The structure of auroral luminosity in the midday sector, Geomagn. Aeron., 28, 256-261, 1988a (in Russian).

Vorobjev, V. G., Zverev, V. L., Starkov, G. V., and Feldstein, Y. I.: Dayside aurora dynamics in dependence on IMF and magnetic activity, Geomagn. Aeron., 28, 251-255, 1988b (in Russian).

Vorobjev, V. G., Khviyusova, T. A., and Starkov, G. V.: Relation of ionospheric parameters to the daytime aurora characteristics, Geomagn. Aeron., 30, 62-67, 1990 (in Russian). 
Vorobjev, V. G., Zverev, V. L., and Starkov, G. V.: Fading of dayside aurorae before the beginning of substorm expansive phase, Geomagn. Aeron., 32, 71-75, 1992 (in Russian).

Vorobjev, V. G., Leontyev, S. V., and Feldstein, Ya. I.: Extended period of polar cap auroral display: auroral dynamics and relation to the IMF and the ionospheric convection, Ann. Geophys., 13, 854-862, doi:10.1007/s00585-995-0854-9, 1995a.

Vorobjev, V. G., Zverev, V. I., and Feldstein, Y. I.: Polar cap auroras: morphological features and relation to $\theta$-auroras, Geomagn. Aeron., 35, 34-43, 1995b.

Wallis, D. D., Anger, C. D., and Rostoker, G.: The spatial relationship of auroral electrojet and visible aurora in the evening sector, J. Geophys. Res., 81, 2857-2869, 1976.

Whalen, J. A., Buchau, J., and Wagner, R. A.: Airborne ionospheric and optical measurements of noontime aurora, J. Atmos. Terr. Phys., 33, 661-678, 1971.

White, F. W. G. and Geddes, M.: The antarctic zone of maximum auroral frequency, Terr. Magn. Atmos. Elect., 44, 367-377, 1939.

Wiens, R. H. and Vallance, J. A.: Studies of auroral hydrogen emissions in west-central Canada. Proton and electron auroral ovals, Can. J. Phys., 47, 1493-1517, 1969.

Wrangel, F. P.: Narrative of an expedition to the Polar Sea in the years $1820,1821,1822, \& 1823$, edited by: Sabine, E., James Madden Company, London, 1840.

Yahnin, A. G. and Sergeev, V. A.: Frequency of aurorae appearances in the polar cap and orientation of IMF, Geomagn. Aeron., 19, 566-571, 1979 (in Russian).

Yahnin, A. G. and Sergeev, V. A.: Polar cap aurora: Dependence on IMF orientation and substorm; certain pecularities of morphology, in Aurorae and airglow, Vol. 28, 27-34, Nauka, Moscow, 1981 (in Russian).
Yahnin, A. G., Malkov, M. V., Sergeev, V. A., Pellinen, R. J., Aulamo, O., Vennerstrom, S., Friis-Christensen, E., Lassen, K., Danielsen, C., Craven, J. D., Deehr, C., and Frank, L. A.: Features of steady magnetospheric convection, J. Geophys. Res., 99, 4039-4051, 1994.

Yahnin, A. G., Sergeev, V. A., Bösinger, T., Sergienko, T. I., Kornilov, I. A., Borodkova, N. L., Thomsen, M. F., Belian, R. D., Sauvaud, J.-A., Lutsenko, V. N., and Skalsky, A. A.: Correlated Interball/ground-based observations of isolated substorm: The pseudobreakup phase, Ann. Geophys., 19, 687-698, doi:10.5194/angeo-19-687-2001, 2001.

Zhang, Y., Paxton, L. J., Morrison, D., Lui, A. T. Y., Kil, H., Wolven, B., Meng, C.-I., and Christensen, A. B.: Undulations on the equatorward edge of the diffuse proton aurora: TIMED/GUVI observations, J. Geophys. Res., 110, A08211, doi:10.1029/2004JA010668, 2005.

Zverev, V. L., Starkov, G. V., Vorobjev, V. G., Leontiev, S. V., Feldstein, Y. I., Elphinstone, R. D., Murphree, J. S., and Cogger, L. L.: Simultaneous observations of auroras in northern and southern polar caps, Geomagn. Aeron., 32, 62-63, 1992.

Zverev, V. L., Feldstein, Y. I., and Vorobjev, V. G.: Visual luminosity equatorwards of the auroral oval during magnetic storms, in: Proceedings of the XXXII. Annual Seminar, Apatity, Physics of Auroral Phenomena, Vol. 32, edited by: A. G. Yahnin, 48-51, Kola Science Centre, Russian Academy of Science, 2009.

Zverev, V. L., Feldstein, Y. I., and Vorobjev, V. G.: Auroral luminosity equatorward of the auroral oval, Geomagn. Aeron., 52, 64-72, 2012. 